\title{
Combined Finite Element (FE) Modeling and Fluid Shear Experiment to Determine the Viscoelastic Material Properties of Osteocytes
}

\author{
J. Qiu ${ }^{2}$, A.D. Baik ${ }^{1}$, X.L. Lu ${ }^{1}$, Z. Zhuang ${ }^{2}$, X.E. Guo ${ }^{1}$ \\ ${ }^{1}$ Department of Biomedical Engineering, Columbia University, 1210 Amsterdam Avenue, New York, NY, 10027 \\ ${ }^{2}$ School of Aerospace, Tsinghua University, Beijing, P.R. China
}

\begin{abstract}
Osteocytes exhibit solid-like viscoelastic behavior in response to mechanical stresses. The goal of this study was to determine the viscoelastic properties of osteocytes using a combined finite element analysis (FE) modeling and experimental approach. The three-dimensional (3D) cell shape of the osteocyte under fluid flow was reconstructed using using a novel pseudo-3D microscopy technique. The cell shape was input into an ADINA fluid-structure FE software. The osteocyte was modeled using a linear and incompressible viscoelastic standard solid with a finite strain. The viscoelastic material parameters were determined by matching the predicted cell surface displacements with those measured experimentally. The instantaneous modulus of an osteocyte was $0.81 \mathrm{kPa}$ and the equilibrium modulus was $0.11 \mathrm{kPa}$. The apparent viscosity were $0.85 \mathrm{kPa}$-s. The material properties measured in this study are comparable to the cell material properties reported in previous studies. This fluid-structure interaction cell model based on individual cell geometry may provide a novel technique to measure the viscoelastic properties of individual cells, as well as potential mechanisms of mechanical signal transduction.
\end{abstract}

\section{INTRODUCTION}

Several techniques have been employed to determine the mechanical properties of single cell, including micropipette aspiration, cell compression within a gel matrix, unconfined cell compression, or various forms of cellular indentation, including cell poking, cytoindentation, or atomic force microscopy (AFM) [1]. Here, a novel technique to determine cell mechanical properties is presented.

\section{MATERIALS AND METHOD}

\section{A. Osteocyte Culture Conditions}

MLO-Y4 osteocytic cells were transfected with EGFP-actin or EMAP-3X-EGFP [2]. The cells were plated for less than 60 minutes on fibronectin-coated glass microslides and stained with Alexa Fluor 594 wheat germ agglutinin plasma membrane dye immediately before flow. Low plating time ensured adherent semi-spherical cells to mimic their in vivo shape.

\section{B. Custom-built Pseudo-3D Microscope System}

A custom-built microscope (Fig.1) was used to obtain two orthogonal images of an osteocyte. An Olympus BX-2 microscope was used for "side-view" images and an Olympus
IX-71 was used for "bottom-view" images. A mirror was aligned at $45^{\circ}$ in the light path of the BX-2 microscope to capture a side-view image.

\section{Shear Flow Experiments}

The glass microslide was placed in a square glass microtube to form a flow chamber. Steady laminar flow with 10 dynes $/ \mathrm{cm}^{2}$ wall shear stress was applied. A single osteocyte was imaged simultaneously by both microscopes with the cytoskeletal GFP and red plasma membrane dyes being imaged sequentially for a combined imaging frequency of $12 \mathrm{~Hz}$.

\section{Image Analysis and 3D Cell Shape Reconstruction}

The cell shape was reconstructed using the bottom-view and side-view images to obtain an accurate cell geometry. The two simultaneously obtained views of the plasma membrane of an osteocyte cell are shown in Fig.2(A) and Fig.2(B). Fig.2(C) shows a typical cell profile after a smoothing and initializing procedure using MATLAB (MathWorks, Natick, MA). Fig.2(D) is the cell profile after fitting using a spline function in Solidworks (Dassault Systèmes, Concord, MA). Fig.2(E) is the cell structure after lofting in Solidworks. Finally, the 3D cell shape in Fig.2(F) was reconstructed after importing the para-solid geometric data file to ADINA (ADINA R \& $\mathrm{D}$, Watertown, MA).

\section{E. Fluid Structure Interaction Finite Element Model}

In the fluid-structure interaction in ADINA FE, the fluid

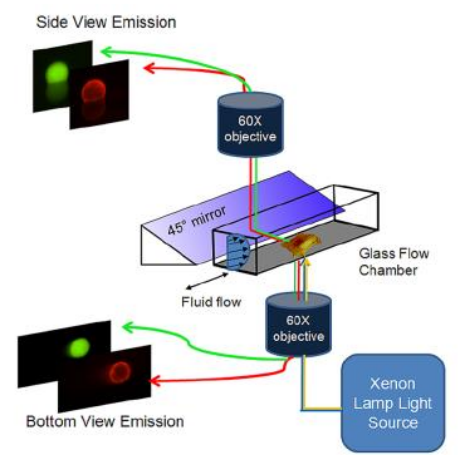

Fig.1. Schematic of light paths of the custom-built dual microscope system and sample cell in both views 


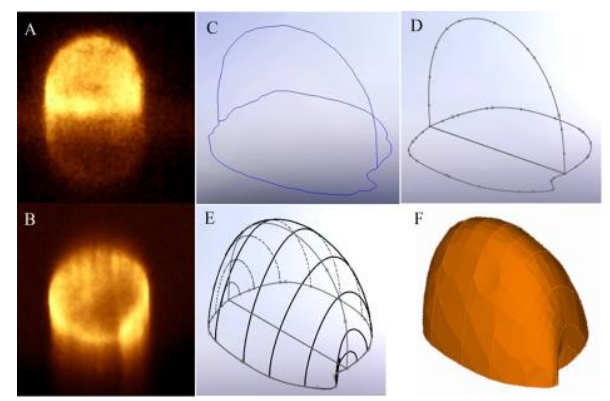

Fig. 2. The process of the cell shape reconstruction.

and solid solution variables are fully coupled. The fluid equation and the solid equation are solved individually in succession, always using the latest information provided from another part of the coupled system [3].

A linear viscoelastic standard solid model was adopted as the constitutive cell material model. There are three parameters in this model, i.e., $\mathrm{k}_{\mathrm{e}}, \mathrm{k}_{1}$, and $\eta$. Here, $\mathrm{k}_{\mathrm{e}}$ is the equilibrium modulus, which determines the equilibrium response of the material, $\mathrm{k}_{\mathrm{e}}+\mathrm{k}_{1}$ represents the instantaneous modulus, which determines the instantaneous response of the material, and $\eta$ is the apparent viscosity, which determines the time to reach the equilibrium state from initial response.

\section{F. Software coupling}

The overall strategy for the simulation [4] is displayed schematically in Fig. 3.

\section{RESULT}

The displacement in Fig.4 is from the apical point of an osteocyte. In Fig.4(A), we found that ostocytes have distinctive displacement mechanical responses due to individual differences of cell material properties.

We choose cell \#3 to determine the cell material properties. There were 5 sets of material parameters. They were divided into 3 groups. Each group had 2 sets of material parameters which only had differences in one parameter.

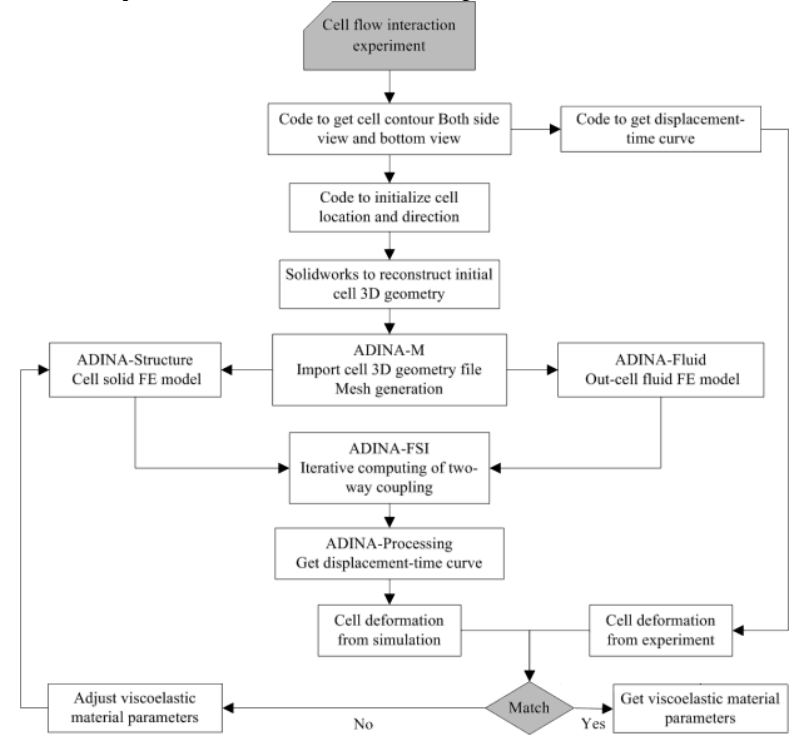

Fig. 3. Schematic representation of the software infrastructure.
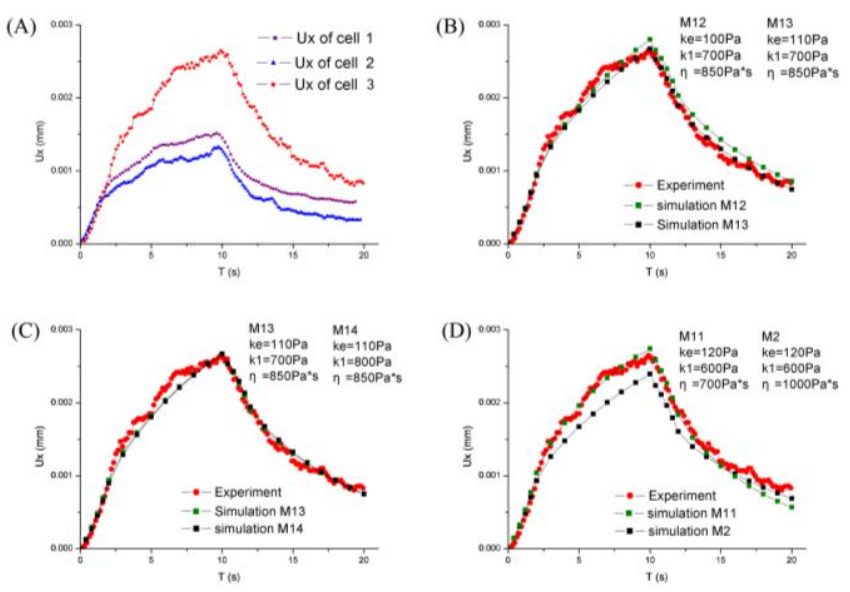

Fig. 4. Displacement-time curve from experiment and numerical simulation.

In Fig.4(B), we slightly increased the equilibrium modulus $\mathrm{k}_{\mathrm{e}}$ from $0.1 \mathrm{kPa}$ to $0.11 \mathrm{kPa}$. Then, the response of displacement significantly decreased. This indicated that $\mathrm{k}_{\mathrm{e}}$ is extremely sensitive to determine the equilibrium modulus using this system.

In Fig.4(C), $\mathrm{k}_{1}$ was increased about $14 \%$, but it only had a small effect on the initial response. There was a difficulty in finding $\mathrm{k}_{1}$, because mimicking or capturing the instantaneous response $\left(\mathrm{k}_{\mathrm{e}}+\mathrm{k}_{1}\right)$ was challenging.

In Fig.4(D), $\eta$ determined the curvature which represented the rate of deformation.

$\mathrm{M} 13$, i.e., $\mathrm{k}_{\mathrm{e}}=0.11 \mathrm{kPa} / \mathrm{k}_{1}=0.7 \mathrm{kPa} / \eta=0.85 \mathrm{kPa}-\mathrm{s}$, was the mechanical property parameters determined by combining the simulation and experiment.

\section{CONCLUSION}

In this work, we have developed a novel technique to determine mechanical properties of a single cell under physiological fluid flow. The enabling component technologies used in the method are simultaneously acquiring two orthogonal profiles of a cell (using the custom-built pseudo-3D microscope system), 3D geometric reconstruction (Solidworks), and fluid structure interaction finite element algorithm (ADINA).

\section{ACKNOWLEDGMENT}

The authors would like to thank QiDa Liu and Bin Zhou for their assistance in programming. This work was supported by an NIH/NIAMS grant RC1-AR058453 and a Fellowship from the Ministry of Eduction of the P.R. China.

\section{REFERENCES}

[1] E. Darling, et al., "Viscoelastic properties of zonal articular chondrocytes measured by atomic force microscopy," Osteoarthritis and Cartilage, vol. 14, pp. 571-579, 2006

[2] K. Faire, et al., "E-MAP-115 (ensconsin) associates dynamically with microtubules in vivo and is not a physiological modulator of microtubule dynamics," Journal of Cell Science, vol. 112, p. 4243, 1999.

[3] K.J. Bathe /ADINA R \& D, Inc. Theory and Modeling Guide Volume III: ADINA CFD \& FSI, 2009, pp.234-235

[4] M. H. Hoskins, et al., "Coupled flow-structure-biochemistry simulations of dynamic systems of blood cells using an adaptive surface tracking method," Journal of Fluids and Structures, vol. 25, pp. 936-953, Jul 2009. 


\title{
Skin Non-Linear Viscoelastic Properties Cannot be Predicted Using Quasi-Linear Viscoelasticity
}

\author{
J.S. Hersey, L.N. Dantzler, J.F. Maher, D.T. Corr \\ Rensselaer Polytechnic Institute \\ $1108^{\text {th }}$ Street \\ Troy, NY 12180
}

\begin{abstract}
The low load nonlinear viscoelastic properties of porcine skin cannot be simplified using the quasi-linear viscoelastic (QLV) model developed by Fung [1].
\end{abstract}

\section{INTRODUCTION}

Normal physiologic loading of collagen based soft tissues generally occurs in the "toe-in" region of the tissue's stressstrain curve. Within the toe-in region the tissue displays a nonlinear strain-stiffening behavior. This increase in stiffness with increasing strain is typically attributed to the progressive uncrimping and recruitment of collagen fibers. In skin the collagen is arranged in a primarily planar array of crimped fibers. While the network contains fibers in all directions, there exists a principal orientation of the fibers within the plane of the tissue, termed Langer's lines, in which the skin is least extensible. When skin is strained, in addition to the uncrimping and progressive recruitment, fibers also rotate to align with the axis of applied loading. This rotation is especially notable when loading oblique to Langer's line [2].

To aid in our understanding of this behavior, theoretical models have been developed to address this complex, nonlinear response. The quasi-linear viscoelasticity (QLV) model developed by Fung [1] has been widely used to simplify the nonlinear viscoelastic behavior of many soft tissues. Within the framework of QLV, a creep response can be used to predict the stress relaxation response, and vice versa. Despite its many successes and wide utility, QLV was shown to be incapable of accurately modeling the low-load behavior of ligament $[3,4]$. In particular, within the toe-in region QLV was unable to predict the creep response from stress relaxation data, and vise versa. The authors attributed this to the presence collagen fiber recruitment during the creep test but lack of recruitment during the stress relaxation test [3].

This study aims to test the applicability of the QLV model to describe the low-load behavior of skin. In particular, we seek to determine if the low load creep response can be predicted from the stress relaxation response, and vice versa. While skin and ligament are both comprised of a network of crimped collagen fibers, in the skin collagen network fibers rotate to align with the axis of loading. We seek to determine if the added effect of fiber rotation in skin may have a different influence on tissue behavior, and on the efficacy of QLV to model physiological, low load skin behavior.

\section{METHODS}

\section{A. Sample Preparation}

Thirty specimens were obtained from dorsal skin sections of female juvenile Yorkshire pigs (Bridge PTS). Each skin section was taken upon sacrifice and frozen for storage and subsequent testing. On the day of testing, the frozen tissue was thawed slowly, equilibrated to room temperature, and all subcutaneous tissue was carefully removed via dissection. Dumbell-shaped test specimens were obtained in the transverse (dorsal-ventral) direction from the skin using a custom designed stainless steel punch. The punch was used to provide consistent geometry, and to avoid stress concentrations that can result from specimen gripping [2]

\section{B. Mechanical Testing}

All mechanical testing was conducted using an INSTRON single column Materials Testing System (Model 5543), equipped with video extensometer (2663 series).

Pilot tests were conducted on 4 samples to characterize the skin's stress-strain behavior. Samples were pre-loaded to 0.1 $\mathrm{N}$ at $15 \mathrm{~mm} / \mathrm{sec}$, then preconditioned for 15 cycles using a constant rate $(10 \mathrm{~mm} / \mathrm{s})$ triangle wave - from $6 \mathrm{~mm}$ grip-to-grip extension to $0.1 \mathrm{~N}$ in load. Finally, the samples were brought to failure at $10 \mathrm{~mm} / \mathrm{sec}$ to generate a corresponding stress-strain curve.

A sample size of 14 was used to generate 14 creep responses and 14 stress relaxation responses corresponding to a specific point in the toe-in region of the stress-strain curve 0.66 strain and $1.37 \mathrm{MPa}$.

The creep tests were run in load control at $16.2 \mathrm{~N}$ (corresponding to $1.37 \mathrm{MPa}$ ). The stress relaxation tests were run in stroke (encoder) control at an extension of $3.67 \mathrm{~mm}$, corresponding to a strain of 0.66 . In each case, creep or relaxation, the tissue was pre-loaded and preconditioned as described in the pilot testing, elongated at a constant rate (10 $\mathrm{mm} / \mathrm{s}$ ) to the target point, and held at the target load or stretch point for a 240 -sec period during which the tissue would creep or relax, respectively.

\section{Regression Analysis}

The normalized stress relaxation and creep responses were analyzed using DeltaGraph ${ }^{\circledR}$ software to generate double exponential equations with five coefficients, as seen in (1) and (2). $\mathrm{G}(\mathrm{t})$ corresponds to the normalized stress relaxation 
response and $\mathrm{J}(\mathrm{t})$ corresponds to the normalized creep response.

$$
\begin{aligned}
\mathrm{G}(\mathrm{t}) & =\mathrm{A}_{1} \mathrm{e}^{\left(\mathrm{A}{ }^{\mathrm{t}}{ }^{2}\right)}+\mathrm{A}_{3} \mathrm{e}^{\left(\mathrm{A}{ }_{4} \mathrm{t}\right)}+\mathrm{A}_{5} \\
\mathrm{~J}(\mathrm{t}) & =\mathrm{B}_{1} \mathrm{e}^{\left(\mathrm{B}^{\mathrm{t}}{ }_{2}\right)}+\mathrm{B}_{3} \mathrm{e}^{\left(\mathrm{B}{ }_{4} \mathrm{t}\right)}+\mathrm{B}_{5}
\end{aligned}
$$

With the relationships expressed in (3), (4), and (5), equations (6) and (7) were used to solve for the corresponding predicted responses $\left(G^{*}(t)\right.$ and $\left.J^{*}(t)\right) . G_{L}(s)$ and $J_{L}(s)$ represent the Laplace transforms of $\mathrm{G}(\mathrm{t})$ and $\mathrm{J}(\mathrm{t})$ respectively.

$$
\begin{aligned}
& \mathrm{G}(0)=1 / \mathrm{J}(0) \\
& \mathrm{G}(\infty)=1 / \mathrm{J}(\infty) \\
& \mathrm{G}_{\mathrm{L}}(\mathrm{s}) \mathrm{J}_{\mathrm{L}}(\mathrm{s})=1 / \mathrm{s}^{2} \\
& \mathrm{G}^{*}(\mathrm{t})=\operatorname{laplace}^{-1}\left(1 /\left(\mathrm{s}^{2} \mathrm{~J}_{\mathrm{L}}(\mathrm{s})\right)\right) \\
& \mathrm{J}^{*}(\mathrm{t})=\operatorname{laplace}^{-1}\left(1 /\left(\mathrm{s}^{2} \mathrm{G}_{\mathrm{L}}(\mathrm{s})\right)\right)
\end{aligned}
$$

The coefficients found in $G^{*}(t)$ and $J^{*}(t)$ were compared to the original $G(t)$ and $J(t)$ coefficients for all 14 samples, respectively. Percent error was calculated and average and standard deviation of the percent errors of each coefficient were also taken.

\section{RESULTS}

The mechanical testing yielded 12 out of 14 viable pairs of stress relaxation and creep responses. The regression analysis provided 24 equations in the double exponential form shown in (1) and (2). These equations were then used to predict the other response yielding 24 prediction equations. The predicted coefficients of each test were compared to the original coefficients of the same test (i.e. predicted creep to original creep and predicted stress relaxation to stress relaxation responses). The results of the analysis are shown in table 1 . Figure 1 is representative of the results found in all 12 samples in the study. Each of the 12 samples illustrated the same pattern of over predicted creep response and under predicted stress relaxation response.

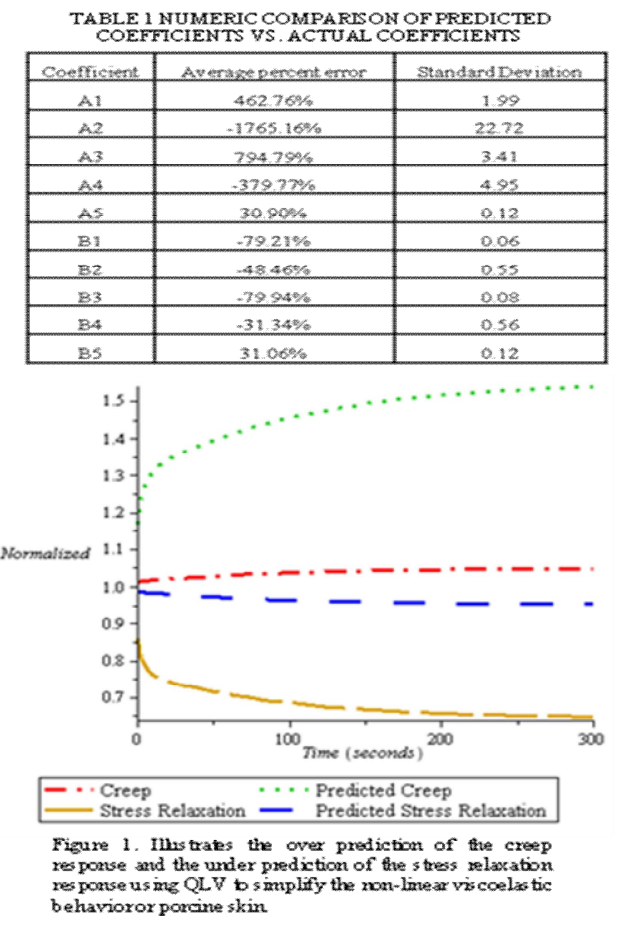

\section{DISCUSSION}

These findings illustrate that QLV is unable to accurately account for the non-linear viscoelastic behavior displayed by porcine skin at low loads. The QLV model predicts a larger creep response and a smaller stress relaxation response than is actually observed during mechanical testing (Fig. 1). Furthermore, there is a significant and relatively consistent difference between the predicted and actual responses (Tab 1.)

These inaccuracies can be explained by the low load nonlinear viscoelastic properties of porcine skin not fitting the requirements for using the QLV method. The QLV method requires that the non-linear viscoelastic material have a time dependent strain history but a constant strain rate dependency [4].

Studies done on other collagenous soft tissues like ligament have shown similar inaccuracies when using the QLV method. The strain stiffening caused by fiber recruitment causes these soft tissues to have an added component to their strain history. As the soft tissue creeps, fibers are continually recruited until all of the fibers are aligned and experiencing a shared tensile force. Thus, the fibers behave as a self-stabilizing network. However, during a stress relaxation test the number of fibers recruited occurs nearly instantaneously during the initial elongation of the material. During the stress relaxation test, the fibers relax causing the characteristic stress relaxation response without recruiting any additional fibers. Since these are two fundamentally different events, the inaccuracies of the QLV model can be explained by taking into account fiber recruitment during creep tests [3].

In skin, in addition to uncrimping, the fibers also rotate to align with the axis of loading. Thus, to be fully engaged and bearing load, the collagen fibers in skin need to rotate to the plane of the force being applied and then uncrimp before they bear a tensile load. Like ligament, skin experiences fiber recruitment during creep but no recruitment during stress relaxation. Therefore the finding that the QLV model is inaccurate in predicting the low load creep of skin from low load stress relaxation and vice versa, agrees with previous findings in ligament [3]. While this study finds the QLV model ineffective in predicting low load creep from low load stress relaxation and vice versa, future studies will have to determine if QLV holds for other areas of the stress-strain curve.

\section{REFERENCES}

[1] Y.C. Fung, Biomechanics: Mechanical Properties of Living Tissues, $2^{\text {nd }}$ ed., New York: Springer, 1993 pp. 277-292

[2] D.T. Corr, C.L. Gallant-Behm, N.G. Shrive, and D.A. Hart "The Biomechanical Behavior of Scar Tissue and Uninjured Skin in a Porcine Model," Wound Rep Regen, vol17, pp. 250-259, 2009.

[3] G.M. Thornton, A. Oliynyk, C.B. Frank, and N.G. Shrive "Ligament Creep Cannot be Predicted from Stress Relaxation at Low Stress: A Biomechanical Study of the Rabbit Medial Collateral Ligament," Journal of Orthopedic Research, vol. 15, No. 5 pp. 652-656, 1997.

[4] P.P. Provenzano, R.S. Lakes, D.T. Corr, R. Vanderby Jr "Application of nonlinear viscoelastic models to describe ligament behavior," Ann. Biomed. Eng. vol 29, pp. 908-914, 2001 


\title{
Effect of Enzymatic Digestion on the Transverse Mechanical Properties of Annulus Fibrosus Lamellae
}

\author{
J.L. Isaacs, MS; E. Vresilovic, MD/PhD; M. Marcolongo, $\mathrm{PhD}$ \\ Drexel University \\ $31^{\text {st }}$ and Market Sts \\ Philadelphia, PA 19104
}

\begin{abstract}
Intervertebral disc (IVD) degeneration is associated with changes in the major biomolecular components in the disc as well as changes in enzymatic activity in the IVD. Collagen, elastin, and proteoglycans in the disc are primarily responsible for mechanical and structural support as well as disc recovery after loading. In order to investigate the effects of the loss of these biomolecules, a single annulus fibrosus (AF) lamellar model has been developed. Isolated segments of human AF were digested with elastase, collagenase and chondroitinase $\mathrm{ABC}$ to selectively degenerate collagen, elastin, and proteoglycan respectively. Samples were then mechanically tested to determine the contribution of these components to the mechanical properties. Understanding the effects of biomolecular changes in the IVD will promote understanding of the relationship between composition and mechanical behavior.
\end{abstract}

\section{INTRODUCTION}

Intervertebral discs (IVDs) stabilize and maintain the alignment of the spine, giving it flexibility by allowing the movement between vertebrae and are responsible for the load distribution and energy absorption under spine loading. They are composed of three distinct parts: cartilaginous endplate, annulus fibrosus (AF), and nucleus pulposus (NP)

The AF is composed of $\sim 25$ lamellae of unidirectional collagen fibers with $\sim 40$ collagen bundles/lamella [1]. Consecutive lamellae run in opposite directions with fibers at an angle of $60^{\circ}$ to the long axis of the spine. The main macromolecular components of the AF are fibrillar collagens, proteoglycans (PG, aggrecan) and elastin. The large-scale organization of elastin fibers in the IVD has been examined by $\mathrm{Yu}$ et al. [3] in bovine tail. They are densely distributed in the region between the lamellae inside the AF and also within lamellae, orientated parallel to the collagen fibers [3]. The IVD experiences irreversible chemical and structural changes due to aging. Olczyk [4-6] correlated the changes in IVD collagen, elastin and PG with age. The biochemical compositional changes in the disc alter mechanical behavior of the disc.

Skaggs et al. [7] investigated the mechanical properties of single non-degenerated AF lamellar specimens in plane with the collagen fibers. Using a strain rate of $0.00009 \mathrm{sec}^{-1}$ with grade I and II samples, they obtained samples with an average gauge dimension of $2.9 \times 1.51 \times 0.63 \mathrm{~mm}$. Skaggs' failure stresses and strains of single lamella AF specimens varied by anatomic region ranging from $3.6 \pm 2-10.3 \pm 8.4 \mathrm{MPa}$ for ultimate tensile strength and $9.2 \pm 3.4-15.4 \pm 3 \%$ for strain dependant on anatomical region. Holzapfel et al. [8] also investigated single lamellar mechanics of the human lumbar AF. Samples $(0.4 \mathrm{~mm}$ thick) were tested in $0.15 \mathrm{~mol} / \mathrm{L} \mathrm{NaCl}$ at $37^{\circ} \mathrm{C}$. They calculated the elastic modulus at three locations: low, medium and high. The low values ranged from $3.8 \pm 2.6$ to $8.01 \pm 6.5 \mathrm{MPa}$ and the high values $27.5 \pm 12.8$ to $64.8 \pm 48.6$ $\mathrm{MPa}$ depending on region.

To understand the mechanical consequences of these degenerative changes, we have developed a single lamellar model under different degenerative conditions to test annulus lamellar biomechanics in plane to the collagen and elastin fiber directions using a micro-mechanical test protocol and post processing micro-strain analysis.

\section{METHODS}

\section{A. Sample Preparation and Enzyme Digestion}

IVDs from human cadaveric samples were obtained from NDRI (Philadelphia) and the posterior elements removed. Single lamellar $(150 \mu \mathrm{m}$ x $5 \mathrm{~mm} \times 15 \mathrm{~mm})$ lumbar AF samples were cut using a Leica 3050 S cryostat with a tungsten carbide blade. The samples were placed into four groups and digested using the following protocol. Samples were places in $5 \mathrm{~mL}$ of buffer for two hours at room temperature, followed overnight by $5 \mathrm{~mL}$ of buffer and the respective enzyme at $37^{\circ} \mathrm{C}$. After the enzyme soak, they were placed in $5 \mathrm{~mL}$ of $1 \mathrm{x}$ PBS at $5^{\circ} \mathrm{C}$ for 1 hour. Prior to testing, the samples were allowed to reach room temperature in $1 \mathrm{x}$ PBS.

Table One: Buffer and Enzyme Components for Sample Groups

\begin{tabular}{|c|c|c|c|}
\hline Group & $\mathrm{pH}$ & Enzyme & Buffer \\
\hline Control & 7.4 & - & $1 \mathrm{x}$ PBS \\
\hline ChABC & 8.0 & $\begin{array}{c}\text { Chondroitinase ABC } \\
(0.125 \mathrm{U} / 5 \mathrm{~mL})\end{array}$ & $\begin{array}{c}0.02 \% \mathrm{BSA}, 50 \mathrm{mM} \text { TRIS, } \\
60 \mathrm{mM} \text { Sodium Acetate }\end{array}$ \\
\hline COL & 7.4 & $\begin{array}{c}\text { Collagenase } \\
(10 \mathrm{U} / 5 \mathrm{~mL})\end{array}$ & $\begin{array}{c}50 \mathrm{mM} \mathrm{TES} \\
2 \mathrm{mM} \mathrm{CaCl}\end{array}$ \\
\hline ELA & 8.5 & $\begin{array}{c}\text { Elastase } \\
(10 \mathrm{U} / 5 \mathrm{~mL})\end{array}$ & $0.1 \mathrm{M}$ TRIS \\
\hline
\end{tabular}

B. Micro-Mechanical Testing

Sample grips were made using two $3 \times 1 \mathrm{~cm}$ strips of $3 \mathrm{M}$ Wetordry ${ }^{\circledR}$ very fine sandpaper. Viable AF samples exhibited unidirectional fibers. The sandpaper enclosed sample was secured into the microtensile testing device parallel to fiber direction. The samples were preconditioned to $\varepsilon=0.1$ for five cycles then loaded until failure (strain rate $0.02 \mathrm{~mm} / \mathrm{sec}$ ). 
Engineering stress (force divided by undeformed crosssectional area at equilibrium hydration) versus strain (the instantaneous length divided by the starting length) was plotted for each specimen. Initial modulus (tangent to the toe region of the response), ultimate modulus (tangent to the second linear region of the response) strain energy (area under the curve) and failure strain were calculated from the raw data.

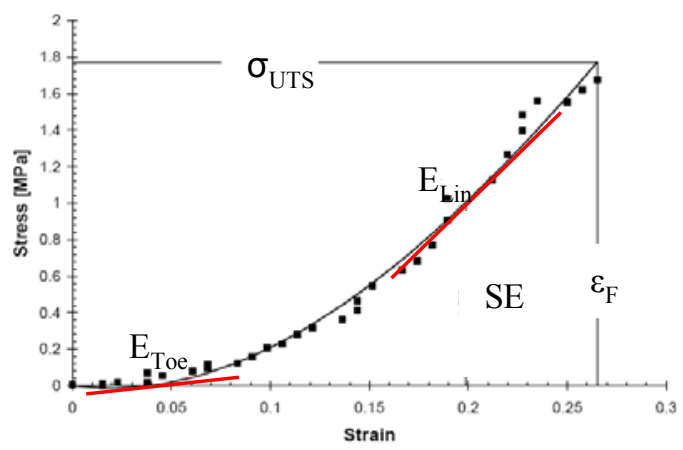

Figure One: Representative stress-strain curve of an in-plane single layer $\mathrm{AF}$ illustrating failure stress $\left(\sigma_{\mathrm{UTS}}\right)$, failure strain $\left(\varepsilon_{\mathrm{F}}\right)$, strain energy $(\mathrm{SE})$ and linear and toe elastic modulus $\left(\mathrm{E}_{\mathrm{Lin}}, \mathrm{E}_{\mathrm{Toe}}\right)$

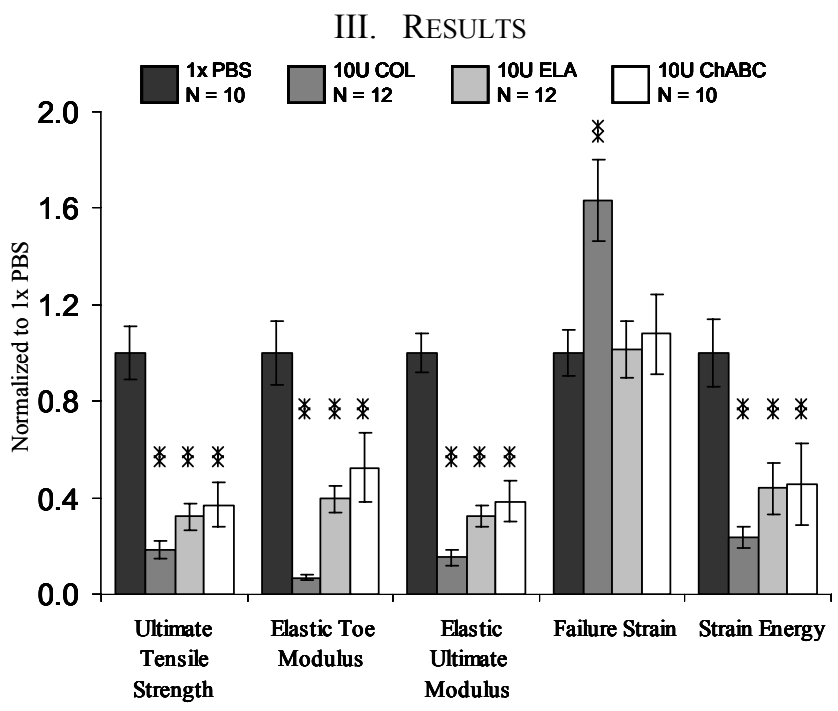

Figure Two: Enzyme effect on properties of individual AF lamellae parallel to COL fibers. Data normalized to $1 x$ PBS. 迷 $p<0.005$ from $1 \times$ PBS.

The control group had an ultimate tensile strength (UTS) of $2.37 \pm 0.26 \mathrm{MPa}$. The enzymatically digested groups showed a significant decrease in UTS. COL samples decreased to 0.43 $\pm 0.09 \mathrm{MPa}$, while the ELA and ChABC groups decreased to $0.77 \pm 0.14$ and $0.88 \pm 0.22 \mathrm{MPa}$, respectively. The control group had a strain failure $\left(\varepsilon_{\mathrm{F}}\right)$ of $0.19 \pm 0.02$ with only $\mathrm{COL}$ showing a significant difference from the control.

A third-order polynomial was fit to the tensile stress-strain behavior based on Skaggs et al. [7]. As with many collagenous tissues, the stress-strain response of the single lamellar AF specimens exhibited nonlinear behavior. The toe region for the specimens occurred at strains under $10 \%$ and at higher strains there was an ultimate linear region.

The elastic toe and linear moduli $\left(\mathrm{E}_{\mathrm{Toe}}, \mathrm{E}_{\mathrm{Lin}}\right)$ for the control group was found to be $5.33 \pm 0.71$ and $17 \pm 1.32 \mathrm{MPa}$, respectively. The COL samples decreased to $0.37 \pm 0.05$ and $2.58 \pm 0.55 \mathrm{MPa}$, while the ELA and ChABC groups decreased to $2.19 \pm 0.33,5.68 \pm 0.82 \mathrm{MPa}$ and $2.8 \pm 0.77$, $6.52 \pm 1.42 \mathrm{MPa}$, respectively.

The strain energy (SE) of the control samples was found to be $0.18 \pm 0.03 \mathrm{~J} / \mathrm{m}^{3}$. The COL samples decreased to $0.04 \pm$ $0.01 \mathrm{~J} / \mathrm{m}^{3}$; the ELA and ChABC groups decreased to $0.08 \pm$ 0.02 and $0.11 \pm 0.04 \mathrm{~J} / \mathrm{m}^{3}$, respectively.

\section{DISCUSSION}

Our data for UTS and $\varepsilon_{\mathrm{F}}$ of the control samples were in the range from literature [7]. The values for $\mathrm{E}_{\mathrm{Toe}}$ and $\mathrm{E}_{\mathrm{Lin}}$ of the control samples were also in the range of the low and high moduli reported in the literature [8].

A limitation in the sample preparation allows the introduction of edge effects into the mechanical properties [10], however, the properties described here provide insights into the intralamellar mechanical behavior, which may allow further understanding of alterations in mechanics seen with disc degeneration.

The absolute values of the mechanical properties for all the groups, enzyme and buffer are lower than that of the control group. The COL samples reduced the UTS by $80 \%$ from the non-degraded group, indicating the importance of collagen to the tensile strength of the annulus fibrosus. Both the ELA and ChABC sample groups resulted in a reduction of the UTS by about $65 \%$. This suggests that elastic fibers function to guide and restrain the deformation of the PG matrix and that on their removal the collagenous elements may play an even more dominant role in the tissue mechanics.

In the literature, degeneration causes a reduction in UTS while $\varepsilon_{\mathrm{F}}$ stays constant with values of approximately $20 \%$ in the circumferential direction [9]. The COL samples showed an increase in the $\varepsilon_{\mathrm{F}}$ as compared to the control. By removing the collagen fibers the elastin fibers were the load bearing elements and the properties reflect that of elastin which has a higher $\varepsilon_{F}$ than collagen.

\section{ACKNOWLEDGEMENT}

We would like to thank T. Shear, P. Marcum and K. Reaser at University of Pennsylvania Veterinary Lab for sample preparation and $\mathrm{M}$. Barsoum for use of the microtensile device.

\section{REFERENCES}

1] Matsui, H., et al. Spine, 1998. 23(9): p. 1029-34.

[2] Adams, M.A. and W.C. Hutton. Spine, 1982. 7(3): p. 184-91. Yu, J., et al. J Anat, 2002. 201(6): p. 465-75. Olczyk, K. Zeitschrift für Rheumatologie. 53(1): p. 19. Olczyk, K. Polish Academy of Sciences, 1994. 32(1): p. 41. Olczyk, K . GERONTOLOGY-BASEL-, 1992. 38: p. 196-196. Skaggs, D.L., et al. Spine, 1994. 19(12): p. 1310-1319. Holzapfel, G., et al. Biomech Model Mechanobiol, 2005. 3(3): p. 125-40. Acaroglu, E.R., et al. Spine, 1995. 20(24): p. 2690.

[10] Herakovich, C.T. 1997, New York: Wiley 480 


\title{
Improvement Of Root Canal X-ray Imaging By Delmopinol Pretreatment-Assisted Contrast Media Infiltration
}

\author{
Satya C. Nayak, Robert E. Baier, Anne E. Meyer, Tariq Abuhaimed \\ The State University of New York at Buffalo, School of Dental Medicine \\ Buffalo, New York, 14214
}

\section{Objective:}

To apply surface-active biofilm-penetration aids to uninstrumented tooth root canals to provide better infiltration of x-ray contrast media and development of digital images that would assist in instrumentation as well as identification of smaller channels present.

\section{Introduction:}

Teeth that require endodontic treatment often present with multiple root canals having complex morphologies difficult to navigate with the aid of only conventional xrays. Previous attempts to provide better images of these structures by pressure- or vacuum-applied contrast media, in pre-cleaned root canals, produced mixed results unlikely to support such applications in biofilmcompromised natural un-instrumented roots $[1,2,3]$. Recent advances in development of biofilm-collapsing oral rinses [4], digital radiography, and desktop image analysis tools encouraged this new investigation of surfactant-assisted contrast media penetration of uninstrumented root canals, examining the outcomes by before-and-after image subtraction criteria for extracted human teeth viewed at 0 vs 30 degree angulations to the $\mathrm{X}$-ray beam.

\section{Study Design:}

The study used a crossover design where 13 teeth were radiologically examined as-received and after access opening of the pulp chambers, before and after application of Reno-60®. The access-opened teeth were water-stored and examined again, and then were divided into two groups, each subjected to either $1 \%$ delmopinol or SADS as a first pretreatment prior to Reno-60® addition and digital radiography at 0 and 30 degree angulations. After Reno- $60 \AA$ removal, the teeth were subjected to second pre-treatments by the alternative reagent and then Reno- $60 \AA$ re-applied prior to additional radiography. All radiographic images were digitally stored and then subjected to pair-wise digital subtractions using Adobe PhotoShop CS for comparison of visualized canal areas. Results were statistically examined using SPSS software and applied t-tests.

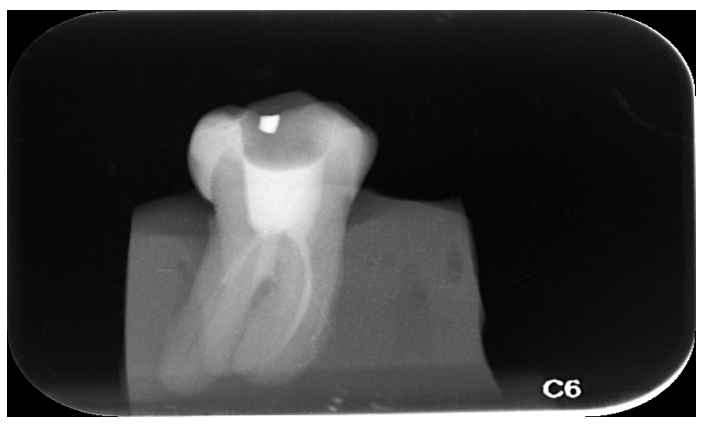

Figure 1: Reno-60 at $0^{0}$ angulation

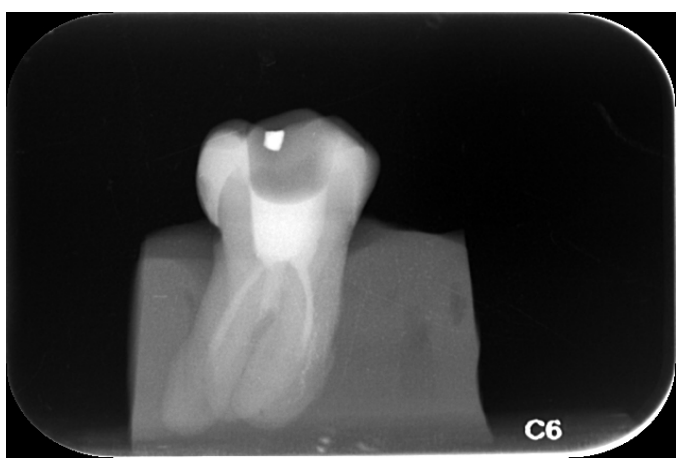

Figure 2: $1 \%$ delmopinol pretreated at $0^{0}$ angulation (increased penetration of Reno-60)

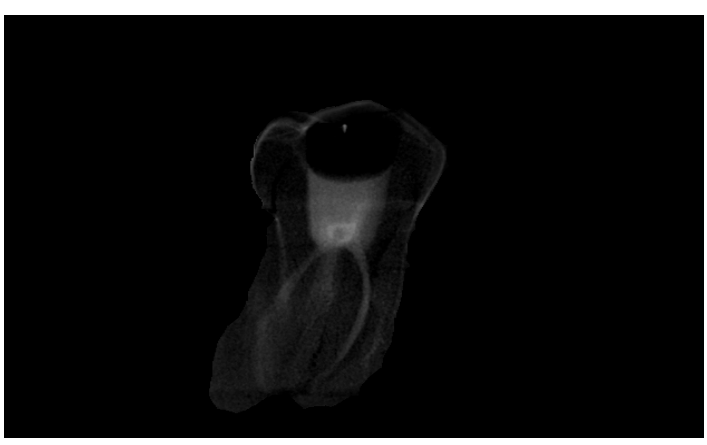

Figure 3: Subtraction image (Using Adobe ${ }^{\circledR}$ Photoshop ${ }^{\circledR)}$ 


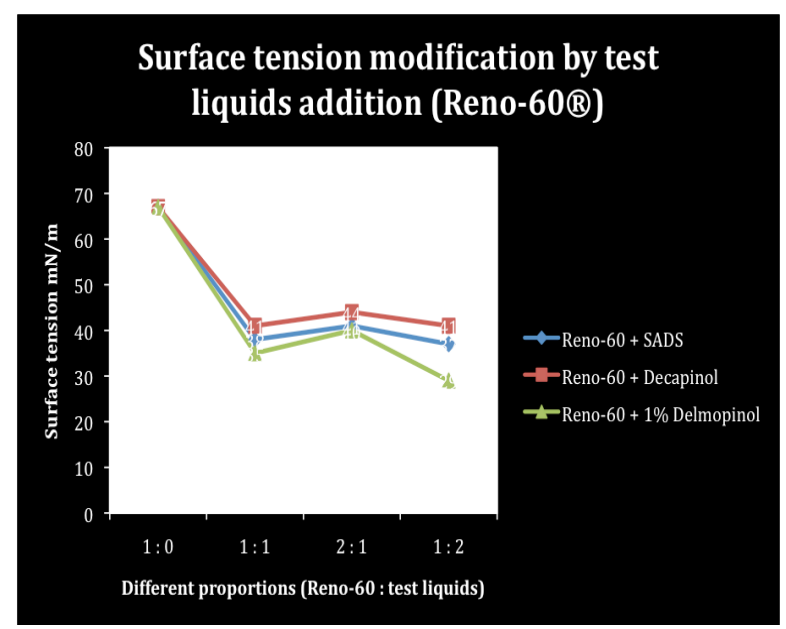

Figure 4: Contact angle analysis

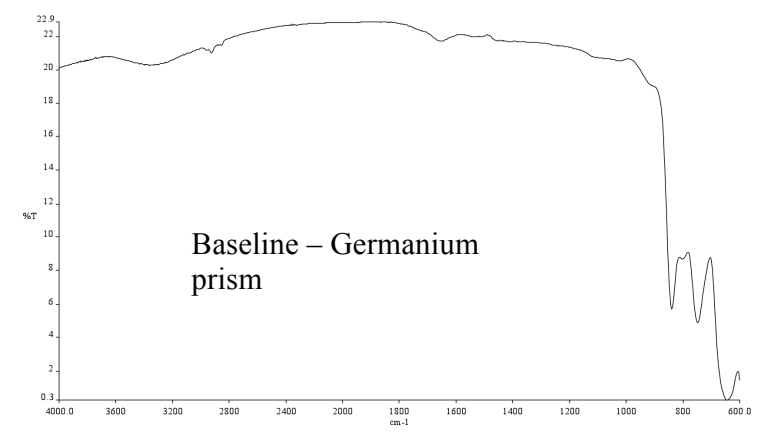

Figure 5: Infrared spectroscopic analysis Reno- $60^{\mathrm{TM}}$

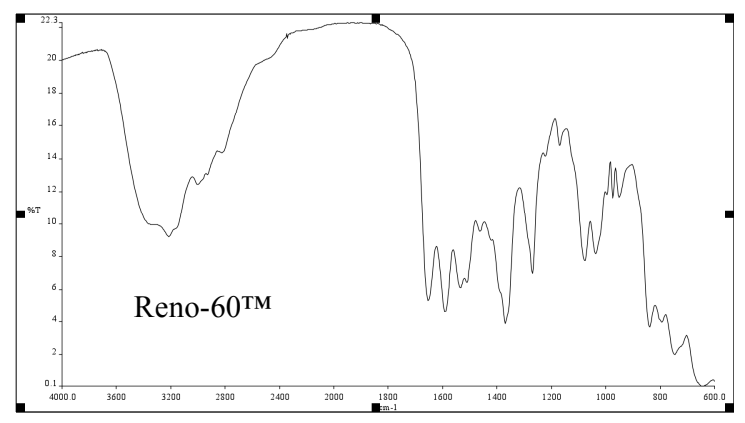

Figure 6: Infrared spectroscopic analysis Reno- $60^{\mathrm{TM}}$ air-dried

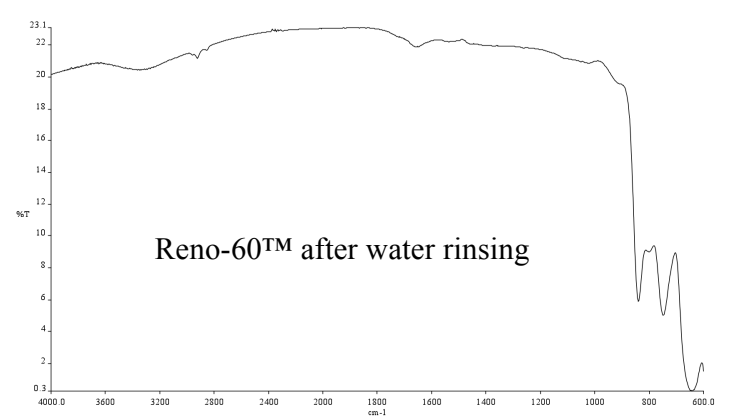

Figure 7: Infrared spectroscopic analysis Reno- $60^{\mathrm{TM}}$ water rinsed

\section{Results:}

Statistically significant differences were found favoring $1 \%$ delmopinol pretreatment as a contrast media penetration aid for root canals, with even better results after a secondary application of SADS in confirmation of earlier cleaning studies where SADS augmented cleaning effectiveness of other irrigant solutions.

\section{Conclusions:}

The teeth pre-treated with $1 \%$ delmopinol followed by SADS showed significantly improved contrast medium penetration in comparison with all other treatments. Pretreatment with 1\% delmopinol was able to increase the penetration of root canals by the contrast medium and this can be attributed to delmopinol's biofilm dissolution ability and high spreading pressure.

\section{Acknowledgments:}

1. Dr. Eugene Pantera: for providing access to endodontic clinic

2. Dr. Maureen Donley: for digital radiography training

3. Kristy Hammond: for providing access to University at Buffalo, School of Dental Medicine server

4. Barb Benzinger: for Picasso training

\section{References:}

1. J. M. Bedford, D. M. Martin, \& C. C. Youngson. Assessment of a contrast medium as an adjunct to endodontic radiography. International Endodontic Journal, 37, 806-813, 2004. 2004;37:806-13.

\section{A. C. Shearer, F. Wasti, N. H. F. Wilson. The} use of a radiopaque contrast medium in endodontic radiography. International Endodontic Journal 1996;29:95-8.

3. Gunnar Hasselgren, Torsten Stromberg. The use of iodine as contrast medium in endodontic therapy. Oral Surgery Oral Medicine Oral Pathology. 1976;41(6):785-8. 4. Martin Addy, John Moran, and Robert G.

Newcombe. Meta-analyses of studies of $0.2 \%$ delmopinol mouth rinse as an adjunct to gingival health and plaque control measures. Journal of Clinical Periodontology. 2007;34:58-65. 


\title{
Analysis of the Mechanical Behavior of the Lumbar Spine Under High Impact Loading
}

\author{
$+{ }^{1}$ Jamison IV, D; ${ }^{1}$ Cannella, M; ${ }^{2}$ Pierce, E C; ${ }^{3}$ Martin, S K; ${ }^{1}$ Marcolongo, M A \\ $+{ }^{1}$ Drexel University, Philadelphia, PA \\ ${ }^{2}$ United States Special Operations Command, Tampa, FL \\ ${ }^{3}$ Naval Surface Warfare Center, Panama City, FL
}

\begin{abstract}
High speed boat (HSB) crewman in the U.S. navy often suffer from lower back pain and accelerated intervertebral disc (IVD) degeneration, due to the high-G impacts experienced during typical missions, resulting in significant time on limited duty status. The relationship between high impact loads and disc biomechanics is not well understood. We seek to analyze the changes in mechanical behavior, particularly compressive stiffness, of anterior column units (ACU) of the lumbar spine as a function of duration of impact.

Lumbar spine sections from four donors with mean age 63 \pm 8.8 years were used, yielding $n=5$ ACUs. The discs were put under quasi-static loading to simulate normal loading conditions and determine the neutral zone and quasi-static loading stiffness. Each ACU then underwent an impact loading sequence, compressing a distance equal to its neutral zone. Each sequence consisted of six impact loads, with varying duration of compression-relaxation $(10,20,40,80,160$, and $320 \mathrm{~ms}$ ).

The data shows there is an increase in stiffness as the impact duration decreased. The sole exception was the $10-\mathrm{ms}$ impact, which yielded stiffness values smaller than that of the 20- or 40-ms impacts. Stiffness values for all impact durations, however, were significantly higher $(p<0.05$, Student's t-test $)$ than that of the quasi-static conditions.

The data supports our theory that the IVD is more stiff under high impact loading conditions than normal loading, and that this stiffness increases with shortened impact duration. This shows that the highly demanding environment of naval crewman is deleterious to the IVD, and may provide insight into the high incidence of back pain and accelerated disc degeneration for this population.
\end{abstract}

\section{INTRODUCTION}

In the U.S. Naval environment, high speed boat (HSB) crewman often suffer from lower back pain and intervertebral disc (IVD) degeneration, due to the high $G$ impacts experienced while navigating through open seas. In a self-reported study of injuries of HSB operators, $33.6 \%$ of these subjects had suffered a lower back injury [1]. The relationship between these high impact loads and disc biomechanics is not well understood. In this study, we analyze the changes in mechanical behavior, particularly compressive stiffness, of anterior column units (ACU) of the lumbar spine as a function of duration of impact. Understanding the mechanical behavior of the IVD during high impact loads, similar to those experienced by the high speed craft operators and crewman, may lead to an understanding of the degenerative process of the disc in this unique operational scenario.

\section{METHODS}

Fresh frozen lumbar spine sections from four donors (mean age: $63 \pm 8.8$ years, range: $51-70$ years), were used in this study, for a total of $n=5$ ACUs. The discs were thawed, dissected, and potted in Smooth Cast 300 (Smooth-On, Inc, Easton, PA). Anatomical measurements were taken (major and minor diameter, disc height) and the discs were graded to assess the gross morphology [2] (Table 1). The potted ACUs were fit into a custom-made jig [3] in a biaxial servohydraulic dynamic testing system (Model 8874, Instron Corp., Norwood, MA). A preconditioning sequence was run on each disc, in order to restore normal disc mechanics $(50$ cycles at $1 \mathrm{~Hz},-50 \mathrm{~N}$ to $-150 \mathrm{~N}$ compressive load). The discs were then put under a quasi-static loading sequence (sinusoidal waveform, $0.05,0.10$ and $0.50 \mathrm{~Hz}$, $+150 \mathrm{~N}$ to $-1 \mathrm{KN}$ ) for 5 cycles each. From this data, we obtained load-displacement curves, demonstrating the typical viscoelastic response of IVD tissues. From these we determined the neutral zone of each disc.

\begin{tabular}{|c|c|c|c|}
\hline ACU ID & Disc Level & Grade & Neutral Zone (mm) \\
\hline 1 & L1-L2 & 5 & 0.148 \\
\hline 2 & L1-L2 & 2 & 0.200 \\
\hline 3 & L4-L5 & 3 & 0.127 \\
\hline 4 & L2-L3 & 3 & 0.457 \\
\hline 5 & L4-L5 & 3 & 0.157 \\
\hline
\end{tabular}

Each disc then underwent an impact loading sequence, similar to that experienced by HSB crewman, while under a $200 \mathrm{~N}$ compressive preload. Each sequence consisted of six impact loads, with varying duration of load-recovery (10, $20,40,80,160$, and $320 \mathrm{~ms}$ ). The discs were all compressed an amount equal to their neutral zone (see Figure 1). Maximum values for force, acceleration, and stiffness were calculated for each loading sequence using code developed with MATLAB software (The Mathworks, Natick, MA).

\section{RESULTS}

Fig. 2 shows the load imposed on each ACU during impact testing. With the exception of ACU \#1, there is a general trend of increasing force with increased impact duration.

Fig. 3 depicts the relationship between stiffness and impulse duration. All discs show a general trend of increased stiffness with reduced impact duration. 


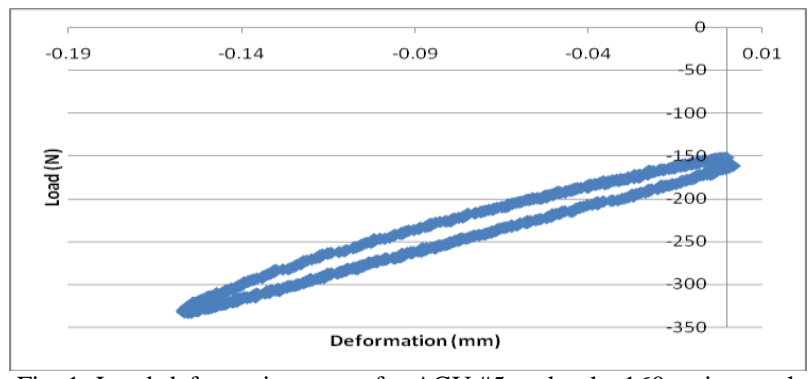

Fig. 1: Load-deformation curve for ACU \#5 under the 160ms impact load. Demonstrates a marked difference in behavior from normal viscoelastic tissues under quasi-static loading conditions.

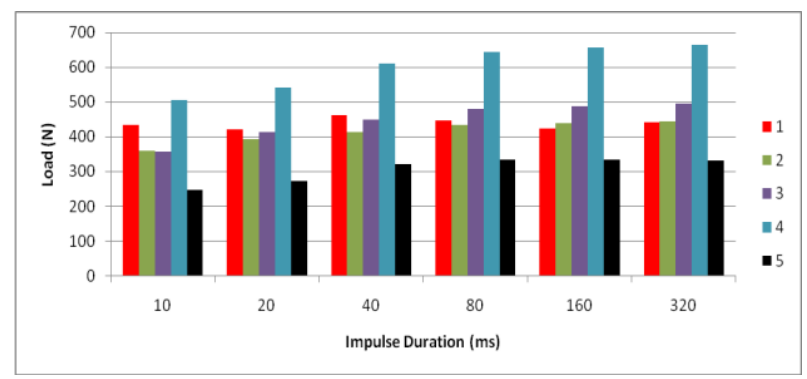

Fig. 2: Compressive Load (+ sense) vs. Impulse Duration. Shows increasing force with increased time of impact loading.

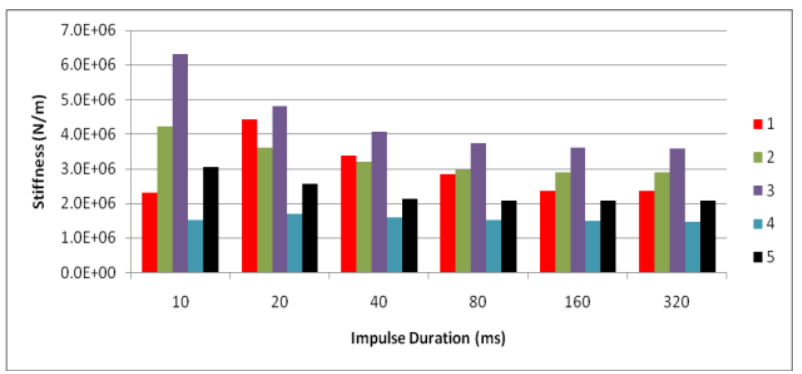

Fig. 3: Stiffness vs. Impulse Duration. Shows increasing stiffness with reduced time of impact loading.

\section{DISCUSSION}

The purpose of this study was to analyze the compressive mechanical behavior of the lumbar spine under high-strainrate impact loads. The data shows that the IVD is more stiff under high impact loading conditions than under quasi-static conditions. The quasi-static compressive stiffness values for intact human motion segments reported in literature vary widely in a range of 700-3200 N/mm [4], and the values reported for the high-impact loading in this study are generally above that range, particularly for the shorter impact loads. In particular, each impact loading stiffness, on average, was significantly higher $(\mathrm{p}<0.05)$ than that of quasistatic laoding for the same samples $(0.84 \mathrm{kN} / \mathrm{mm})$. This shows that the highly demanding environment of naval HSB crewman is taxing on the IVD, and may provide insight into the high incidence of back pain and accelerated disc degeneration for this population.

\section{ACKNOWLEDGEMENTS}

The authors would like to thank the United States Department of Defense for funding and support for this project.

\section{REFERENCES}

[1] Ensign M, et. al., NHRC, 2000

[2] Thomspon JP, et.al., Spine. 15(5), 411-415, 1990

[3] Cannella M, et. al., J Biomech. 41, 2104-2111, 2008

[4] White AA Panjabi, MM, Clinical Biomechanics of the Spine, 1990 


\title{
Tissue-on-tissue lubricity studies of saliva substitutes as- supplied, mixed with and compared with human saliva from control and xerostomia patients
}

\author{
R. Ganesh, R. Baier, A. Meyer \\ University at Buffalo \\ 110 Parker Hall \\ Buffalo, NY 14214
}

\section{INTRODUCTION}

Saliva is one of the most complex and important body fluids [1]. It protects and lubricates oral tissues, provides buffering action and antibacterial activity, helps in maintenance of tooth integrity, and aids in taste perception and digestion [2]. The presence of saliva usually is taken for granted. Nevertheless, its diminution or absence can cause painful tongue and mucosa, problems with taste, swallowing, chewing, and phonation, tooth decay and increased risk for infection [3]. Xerostomia, commonly known as "dry mouth", is a condition caused by diminished salivary flow seen primarily in the elderly experiencing side effects from xerostomic drugs, multidrug interactions, and cancer therapies. About $30 \%$ of the population reports some degree of dry mouth [4], which indicates that it is not an infrequent complaint or patient concern. Management of xerostomia includes maintenance of good oral hygiene, strict adherence to noncariogenic diet and application of topical fluorides [5]. The common clinical approach for these patients is to administer a saliva substitute which functions by moistening the oral cavity to relieve the discomfort caused by oral dryness. The market is flooded with a variety of dry mouth products each claiming to be better than the other. In this study, I have attempted to identify the ingredients of 19 of the saliva substitutes available in the market in order to compare them and see how each ingredient contributes to the efficacy of the product. Unstimulated human saliva may differ in composition and lubricating properties between normal and xerostomic patients and thus their behavior with the saliva substitute admixture. This investigation applied a new tissue-on-tissue lubricity test procedure [6,7], which has shown clinical correlations leading to successful formulations for relieving "dry eye" symptoms, to commercial saliva substitutes as-formulated and after admixture with pre-applied aliquots of unstimulated saliva.

\section{MATERIALS AND MethodS}

The 19 saliva substitutes available for this study were: Aquoral from Auriga Pharmaceuticals, LLC (Lot\# AB0407 0001), Biotene Oral Balance dry mouth moisturizer liquid from Laclede, Inc. (Lot\# 070101), Biotene Oral Balance dry mouth moisturizing gel from Laclede, Inc. (Lot\# 070103),
Bye-Dry mouth moisture lock from Bye-Dry (Professional sample), CariFree Boost oral neutralizer mouth spray from Oral Biotech (Lot\# 290708), Entertainer's Secret throat relief from KLI Corp. (Lot\# 1539), Mouth Kote oral moisturizer from Parnell Pharmaceuticals, Inc. (Lot\# 7108E), Numoisyn Liquid from Align Pharmaceuticals (Lot\# 0906/2), Oasis oral demulcent from GlaxoSmithKline (Lot\# 7D13AC1), Orajel dry mouth moisturizing gel from Del Pharmaceuticals, Inc. (Lot\# 8H55), Rain SPRY dry mouth spray from Xlear Inc. (Lot\# 08204A), Salinum from Biosurface Pharma AB (Lot\# 40249), SalivaSure from Scandinavian Formulas, Inc. (Lot\# 40249), SST from Sinclair Pharma Plc. (Lot\# 6182A), Stoppers4 dry mouth spray from Woodridge Labs, Inc. (Lot\# 11506A), Tom's of Maine mouthwash for dry mouth from Tom's of Maine, Inc. (Lot\# FW1134), Toothette mouth moisturizer from Sage Products Inc. (Lot\# R8641F), Xylimelts from Orahealth Corp. (Professional sample), Xylimelts Mints from Orahealth Corp. (Professional sample).

Compositional analyses were done on Germanium prism using MAIR-IR (Multiple Attenuated Internal Reflection) spectroscopy using Perkin Elmer Spectrum 100 FT-IR spectrometer. Contact angle analysis of each of the substitutes was measured on standard Teflon using Rame-Hart Model Contact Angle Goniometer to determine the operational liquid/vapor surface tensions $\left(\gamma_{\mathrm{lv}}\right)$. $p H$ was measured using Beckman scientific $\mathrm{pH}$ meter and $\mathrm{pHydrion} \mathrm{pH}$ testing paper. Tissue-on-tissue lubricity of the substitutes were compared by measuring the coefficient of friction between surfaces of glutaraldehyde-preserved bovine pericardium on a reciprocating pin-on-disc device, donated by Spire Corporation (Bedford, MA, USA). Friction between the pericardium was monitored via a strain gauge and strip chart recorder system. Calibration with known weights allowed for the measurement of friction forces as a function of pen displacement on the strip chart.

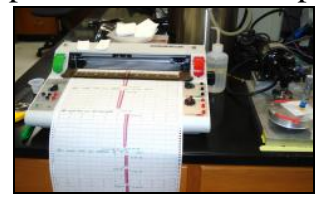

Fig.1:Friction device

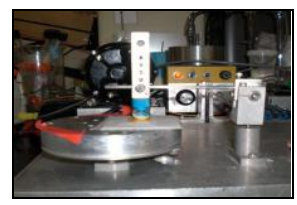

Fig.2:Tissue-on-tissue model 
Under a Human Subjects Institutional Review Board approved protocol (HSIRB \# SIS0531008E), ten normal (control) subjects and ten clinically diagnosed xerostomia patients were asked to donate about $1 \mathrm{ml}$ of unstimulated human saliva for identical sets of lubricity tests and compositional analyses, to establish possible similarities and differences in the level of lubricity in the laboratory model associated with normal vs xerostomic saliva sources and components. The saliva was further tested with 4 saliva substitutes that were chosen based on the results of a pilot study.

\section{RESULTS}

Table 1 shows the $\mathrm{pH}$ and operational liquid/vapor surface tensions of the 19 saliva substitutes and the control Unisol® 4 saline solution supplied by Alcon Research Inc.

\begin{tabular}{|c|c|c|c|c|c|}
\hline Substitute & $\mathrm{pH}$ & $\begin{array}{l}\text { Surface } \\
\text { Tension } \\
\left(\gamma_{m}\right) \\
\mathrm{mN} / \mathrm{m}\end{array}$ & Substitute & $\mathrm{pH}$ & $\begin{array}{l}\text { Surface } \\
\text { Tension } \\
\left(\mathrm{H}_{\mathrm{H}}\right) \\
\mathrm{mN} / \mathrm{m}\end{array}$ \\
\hline Aquora1 & 5.00 & 42 & Rain Spry & 7.20 & 40 \\
\hline $\begin{array}{l}\text { Biotene } \\
\text { liçuid }\end{array}$ & 6.59 & 55 & Salinum & 6.70 & 57 \\
\hline Bre-Dry & 5.00 & 42 & SalizaSure & 4.67 & 55 \\
\hline $\begin{array}{l}\text { Curifree } \\
\text { Boost }\end{array}$ & 9.15 & 55 & SST & 4.11 & 54 \\
\hline $\begin{array}{l}\text { Entertainer's } \\
\text { Secret }\end{array}$ & 7.17 & 58 & Stoppers4 & 4.26 & 42 \\
\hline Mouth Kote & 3.37 & 45 & $\begin{array}{l}\text { Torn's of } \\
\text { Mane }\end{array}$ & 4.14 & 44 \\
\hline Nemoisyn & 6.66 & 53 & Toothette & 6.00 & 55 \\
\hline Ossis & 6.13 & 44 & Unisol4 & 7.40 & $\sim 72$ \\
\hline Orajel & 6.00 & 56 & Xylimelts & 5.78 & 58 \\
\hline $\begin{array}{l}\text { Oral Balance } \\
\text { Ge1 }\end{array}$ & 5.00 & 51 & $\begin{array}{l}\text { Xylimelts } \\
\text { Mint }\end{array}$ & 5.54 & 52 \\
\hline
\end{tabular}

Table 1: $\mathrm{pH}$ and critical surface tension

The lubricity tests indicated good lubrication by substitutes namely - Biotene liquid, Numoisyn, Oasis, Orajel, Oral Balance gel, Salinum, Toothette gel, Xylimelts and Xylimelts Mint.

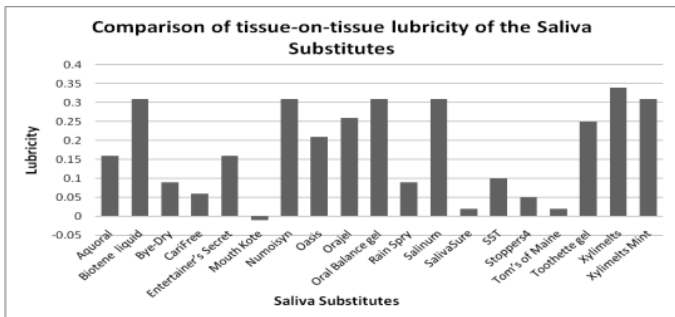

Fig. 3: Tissue-on-tissue lubricity of Saliva Substitutes

Lubricity tests on unstimulated human saliva did not show significant difference when comparing normal vs. xerostomic patients statistically using repeated measures analysis of variance (ANOVA). The substitutes seem to do much better in presence of saliva as compared to lubricity provided when used alone.

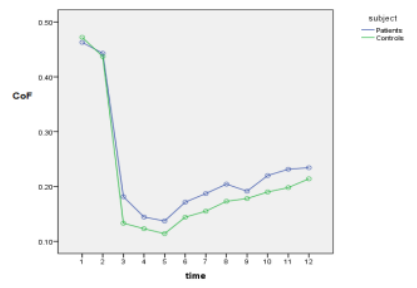

Fig 4: CoF of saliva in Patients vs Controls

\section{DISCUSSION}

The usual complaint of xerostomic subjects is dryness of mouth. This dryness is experienced due to lack of the feeling of wetness caused by saliva. It is important that artificial saliva deals with this issue and provides comfort to patients. The ideal artificial saliva substitute should be long lasting, capable of providing lubrication to wet and protect oral tissues, and able to inhibit the colonization of cariogenic bacteria [8].

The $\mathrm{pH}$ of the saliva substitutes showed variations. It was surprising to see saliva substitutes that were acidic. Increased susceptibility to acid attacks causing demineralization of the tooth surfaces is a major concern in xerostomic patients. To nullify this effect caused by decreased saliva, it would be expected that the saliva substitutes have a neutral $\mathrm{pH}$. Substitutes such as Aquoral, Bye-Dry, Mouth Kote, Oasis, Rain Spry, Stoppers4 and Tom's of Maine were more surfaceactive than the others. The remaining substitutes showed surface tension close to that of saliva.

The lubricity of the saliva alone was compared between the healthy control group and the dry mouth patient group. It was expected that there would be variations in lubricating properties between the 2 groups with the latter performing poorly.

\section{CONCLUSION}

The saliva in dry mouth subjects worked as well as that of the controls in providing lubricity. Their interaction with the saliva substitutes was similar to the behavior of that of the controls with the substitute. Based on the statistical analysis, it is likely that saliva in dry mouth subjects differs from that in normal healthy individuals in quantity alone and not quality.

\section{ACKNOWLEDGMENT}

My sincere thanks to Dr. Michael Hatton for helping me identify and examine subjects for the study.

\section{REFERENCES}

[1] Humphrey SP, Williamson RT. A review of saliva: Normal composition, flow, and function. The Journal of Prosthetic Dentistry. 2001;85:162-9.

[2] de Almeida Pdel V, Gregio AM, Machado MA, de Lima AA, Azevedo LR. Saliva Composition and Functions: A Comprehensive Review. The Journal of Contemporary Dental Practice. 2008;9(3):1-9.

[3] Schenkels LC, Veerman EC, Amerongen AN. Biochemical composition of human saliva in relation to other mucosal fluids. Crit Rev Oral Biol Med 1995;6(2):161-75.

[4] Turner MD, Ship J. Dry Mouth and Its Effects on the Oral Health of elderly people. J Am Dent Assoc. 2007;138:15S-20S.

[5] Guggenheimer J, Moore P. Xerostomia: etiology, recognition and treatment. J Am Dent Assoc. 2003;134:61-9.

[6] Meyer AE, Baier RE, Chen H, Chowhan M. Tissue-on-Tissue Testing of Dry Eye Formulations for Reduction of Bioadhesion. Journal of Adhesion. 2006;82(6):607 - 27.

[7] Meyer AE, Ganesh R, Nayak S. In vitro Tissue-on-Tissue friction model differentiates performance of Dry-mouth products. Poster session presented at the 86th General Session \& Exhibition of the IADR 2008.

[8] Levine MJ, Aguirre A, Hatton MN, Tabak LA. Artificial salivas: present and future. J Dent Res. 1987;66(Spec):693-8. 


\title{
Surface Characterization of Butterfly Wings
}

\author{
N.D. Wanasekara, V.B. Chalivendra \\ University of Massachusetts Dartmouth \\ 285 Old Westport Road \\ North Dartmouth, MA 02747
}

\begin{abstract}
Surface roughness due to micro and nanostructures of butterfly wings create hydrophobic surfaces. In this study, two different experimental techniques were used to characterize the effect of structural features on hydrophobicity of wings. The static and dynamic (advancing and receding) contact angle studies, and Atomic force microscopy (AFM) and optical profilometry characterization were conducted on wings of different butterfly/moth species. Both types of contact angles were shown to be the highest on the butterfly wings that had a combination of both scales and hair like microtrichia. The microtrichia without scales showed almost similar hydrophobicity as on a surface with scales. The role of surface roughness was evaluated by correlating the roughness factor for different scan sizes. It was identified that roughness factor fluctuates rapidly for smaller scan sizes and takes slightly decreasing stable profile for larger scan areas.
\end{abstract}

\section{INTRODUCTION}

Butterflies are well known to have water repellent wings that help them to survive in environments with constant rainfall. The hydrophobicity mechanisms of butterfly wings can be attributed to the sub-micron scale features. Wu et $\mathrm{al}^{1}$ conducted morphological experiments on the wing scales of six butterfly species living in northeast of China. They also presented 3D models considering wing structures obtained from optical microscopy, scanning electron microscopy and transmission electron microscopy. Chen et $\mathrm{al}^{2}$ characterized wettability and self cleaning effect of butterfly wing surfaces using confocal light microscopy. The dust on the surface can easily be cleaned by rolling spherical water droplets when the inclining angle is larger than $3^{\circ}$. It was concluded from their study that nanostructure plays a very important role in the selfcleaning ability. Burton and Bhushan ${ }^{3}$ characterized the adhesion and friction properties of hydrophobic leaf surfaces. Optical profiler and atomic force microscopy were used in the characterization to make the measurements on leaves with and without wax on the surface. It was shown that both micro bumps and wax play an equally important role in the hydrophobic nature as well as the adhesion and friction of leaf.

Our study on wing structures is focused on water wettability and surface roughness characterization. Static and dynamic contact angles of four different species of butterflies were measured to understand the wing hydrophobicity. Atomic Force Microscopic and Optical Profiler were used to characterize and correlate the effect of scan area on surface roughness factor. The selected butterflies are Greta Oto (Glass Wing), Antheraea Polyphemus (Polyphemus moth), Actias Luna (Luna moth) and Phoebis Philea (Oranged-barred sulfur).

\section{EXPERIMENTAL DETIALS}

Initially butterfly wings of around two dozens of different species were collected. All these butterfly wings were subjected to Scanning Electron Microscope Characterization to examine the wing nanostructures. Then five butterflies were selected for further characterization based on distinct surface features of wings. Then static contact angle measurements were carried out using the custom built sessile drop contact angle instrument at University of Rhode Island. The droplet size of $5 \mu \mathrm{l}$ was maintained in all experiments. These measurements were followed by the dynamic advancing and receding contact angle measurements using a high precision goniometer. The direction of wing was maintained as radial outward for all the dynamic experiments. The wings of Phoebis Philea were characterized using Atomic Force Microscope (XE 100, Park Systems Inc., CA, USA). A silicon cantilever with a radius of curvature $<20 \mathrm{~nm}$ was used. The wings were scanned using non-contact (tapping) mode AFM in different sizes ranging from $2 \times 2 \mu^{2}$ to $50 \times 50 \mu^{2}$. Optical profiler (Zygo Corp., CT, USA) was used in imaging large areas.

\section{RESULTS AND DISCUSSION}

The SEM images of selected species are shown in Fig. 1. No scales were found in Greta Oto except for tiny hair like microtrichia coming out of the transparent wing. Phoebis Philea had typical butterfly wing structure with tiled scales all over the wing. The nanostructure of Antherea Polyphemus moth had long fork shape scales which spread with less density. Actias Luna had a combination of low density scales and tiny hair like microtrichia. Fig. 2 shows a comparison between AFM $4 \times 4 \mu m^{2}$ scan size image and optical profiler $350 \times 260 \mu \mathrm{m}^{2}$ scan size image.

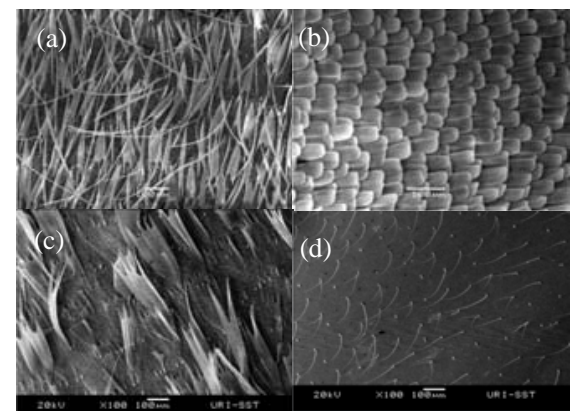

Fig. 1 Photographic images of the butterflies and corresponding SEM images of selected locations. (a)Actias Luna (b)Phoebis Philea (c) Antherea Polyphemus(d) Greta Oto 


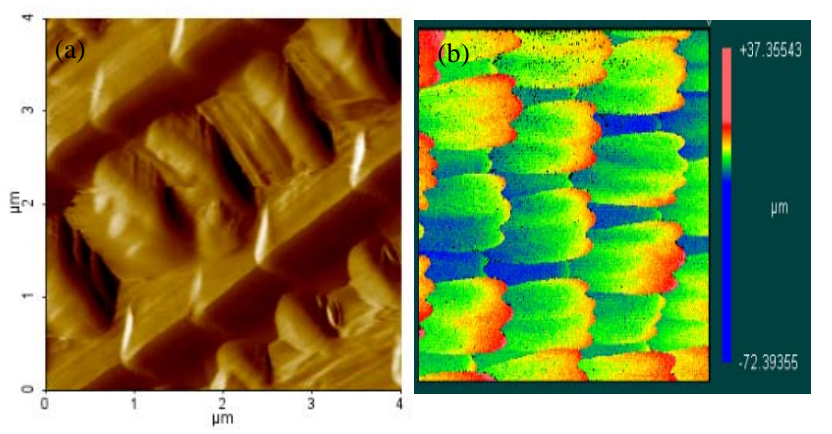

Fig.2 Images showing the nano and micro structures of the 'Phoebis Philea' (a) AFM image of $4 \mathrm{X} 4 \mu^{2}$ (b)optical profilometer image of $350 \mathrm{X} 260 \mathrm{\mu m}^{2}$

Fig. 3 shows the static and dynamic contact angles of selected butterfly sings. The static contact angles measured using sessile drop method are given in Table 1. The static contact angles on butterfly wings represented characteristics of the microstructure. The hydrophobicity of wing structure was proven by the measured contact angles above $100^{\circ}$ except for Antherea Polyphemus. The highest contact angle was measured in Actias Luna which has a wing microstructure of both scales and randomly arranged microtrichia. This suggests that combined structure of scales and microtrichia causes an increase in hydrophobicity. Another important observation was that both Greta Oto and Phoebis Philea have similar static contact angles.

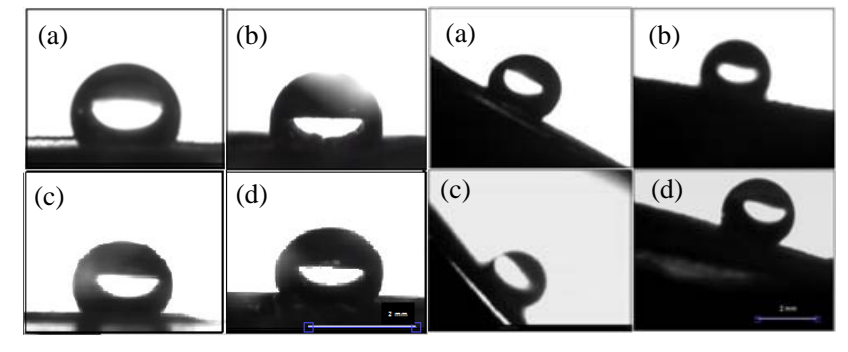

(A)

(B)

Fig.3 (A) Static contact angle images (B) Dynamic contact angle images (a) Greta Oto. (b) Antherea Polyphemus. (c) Actias Luna. (d) Phoebis Philea,

Advancing and receding contact angles on radial outward direction were determined to investigate the response of water droplets on tilting wing surfaces. The measured advancing and receding contact angles of selected four species are given in Table 1. Actias Luna showed the highest advancing angle. Greta Oto and Phoebis Philea have advancing contact angles almost similar to the static contact angles. The microstructure consisting of both tiny microtrichia and scales have the same effect in water rolling over the wing surface in a radial outward direction. Measured receding contact angles of Greta Oto, Antherea Polyphemus and Phoebis Philea were around $90^{\circ}$. That means when the surface was tilted, the receding edge of water droplet deforms significantly before it rolls over.
Table. 1 Static and dynamic contact angles for different butterflies

\begin{tabular}{|l|l|c|c|}
\hline Butterfly Name & $\begin{array}{l}\text { Average } \\
\text { Contact } \\
\text { Angle (deg) }\end{array}$ & $\begin{array}{c}\text { Advancing } \\
\text { Angle(deg) }\end{array}$ & $\begin{array}{c}\text { Receding } \\
\text { Angle (deg) }\end{array}$ \\
\hline Greta Oto & 115.5 & 118.09 & 98.57 \\
\hline Actias Luna & 120.33 & 133.05 & 111.57 \\
\hline Antherea Polyphemus & 94.775 & 116.85 & 97.42 \\
\hline Phoebis Philea & 115.53 & 115.59 & 95.88 \\
\hline
\end{tabular}

The butterfly Phoebis Philea's upper wing surface was scanned with different scan sizes using non-contact mode AFM. The raw data generated from the scanned image was used in calculating the roughness factor, which is defined as the ratio of true surface area to projected area. The roughness factors for different scan sizes are plotted in the Fig. 4. For smaller scan areas, the roughness factor varies between 2 and 9 , since the nano-structured features play a predominant role for such a variation. However, as the scan size increases, the repeating scales as shown Fig. 2(b) provides a stable roughness factor value of 5 .

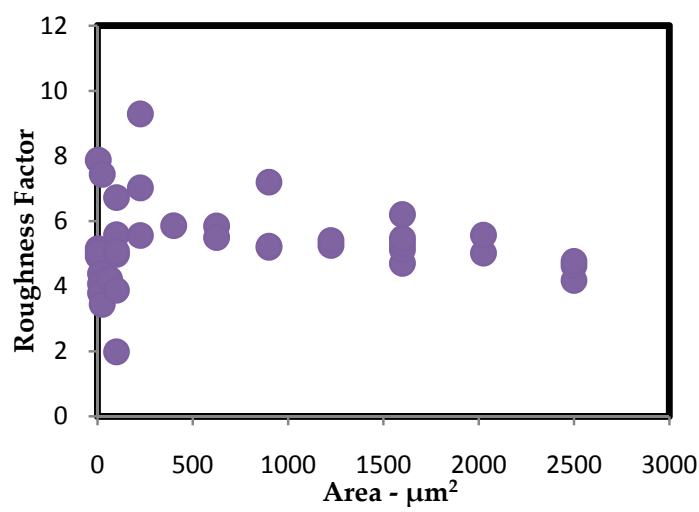

Fig. 4 Variation of roughness factor with scan size

\section{ACKNOWLEDGEMENT}

The authors greatly appreciate the financial support of National Science Foundation through the grant CMS0618119 for acquiring atomic force microscope and National Textile Center for funding through M08-MD13. We would also like to thank Mr. Evan Wujick \& Prof. Arjit Bose of University of Rhode Island for contact angle measurements and also Ms. Chelsea Davis \& Prof. Alfred Crosby at University of Massachusetts, Amherst for optical profilometer measurements.

\section{REFERENCES}

[1] L. Wu, Z. Han, Z. Qiu, H.Guan, L.Ren, “The Microstructures of Butterfly Wing Scales in Northeast of China” J. of Bionic Eng, 2007, 4, 47-52.

[2] G. Chen, Q. Cong, Y. Feng, L. Ren, Institute of Physics Conference Series, 2003, 180, 245-251.

[3] Z. Burton, B. Bhushan, "Surface characterization and adhesion and friction properties of hydrophobic leaf surfaces" Ultramicroscopy, 2006, 106, 709-719. 


\title{
Structure and Elasticity Mechanism of Full Length Resilin Proteins
}

\author{
Xiao $\mathrm{Hu}^{1}$, Guokui Qin ${ }^{1}$, Peggy Cebe ${ }^{2}$, David L. Kaplan ${ }^{1}$ \\ ${ }^{1}$ Department of Biomedical Engineering, ${ }^{2}$ Department of Physics and Astronomy, \\ Tufts University \\ Medford, MA 02155
}

\begin{abstract}
Full length resilin, as well as resilin peptides encoded by exons 1 and 3, were analyzed for structural features. The elasticity mechanisms of resilin related to insect function are described. To approach this goal, a simple energy input method based on thermal treatment was used to detail the transitions and structures of resilin. A systematic pathway to the mechanism was followed based on Temperature-modulated Differential Scanning Calorimetry (TMDSC), Real-time Fourier Transform Infrared Spectroscopy (FTIR), synchrotron Real-time X-ray, and AFM. The results revealed that the conformation and transition of resilin proteins played a critical role for their elastic mechanisms, which provided an effective pathway to design new super elastic biomaterials.
\end{abstract}

\section{INTRODUCTION}

Resilin is an elastic protein found in specialized body regions of many insects, i.e. the jumping organs of arthropods such as froghopper and cat fleas, the wings of dragonflies, or the vibrating membrane of cicadas [1-5]. In Nature, resilin functions as a perfect "super elastic rubber" with more than $90 \%$ elastic efficiency, and can extend and retract millions of times, retaining function over the entire lifetime of the animal [1-5]. The unique resilience properties of resilin proteins, and their potential to become super elastic biomaterials for biomedical applications, have resulted in recent studies [5-14]. However, the molecular structure and transitions of resilin-based proteins remain unclear due to the limited amount of protein that can be collected from insects. Previously, we reported that full length resilin of Drosophila melanogaster was successfully generated using recombinant DNA technology [6], which provided an effective pathway to study in detail study the structure and elastic mechanisms of native resilin protein.

\section{METHODS}

We have previously reported on the recombinant DNA process used to generate full length resilin [6]. The final $1 \%$ aqueous solution of resilin was cast into polystyrene Petri dishes to generate protein films with a thickness of $1 \sim 2 \mu \mathrm{m}$. Films were placed in a vacuum oven at room temperature for 1 day to remove surface moisture. The films, containing $\sim 8 \%$ bound water, were completely unstructured and non-crystalline before thermal treatment. Images were performed in tapping mode on a Dimension 3100 Scanning Probe Microscope with Nanoscope III and IV controllers (Digital Instruments, Santa Barbara, CA) and equipped with rotated tapping-mode etched silicon probes (RTESP; Nanodevices, Santa Barbara, CA).
Protein solution samples were first cast on silicon surfaces to generate thin films. Temperature-modulated Differential Scanning Calorimetry (TMDSC), Real-time Fourier Transform Infrared Spectroscopy (FTIR), and synchrotron Real-time X-ray (Brookhaven National Laboratory (BNL), National Synchrotron Light Source (NSLS) beam line X27C with a wavelength of $0.1371 \mathrm{~nm}$ ) were also used to identify structures and transitions with the protein.

\section{RESULTS}

The recombinant full length resilin of D. melanogaster contains three domains, including: the first exon (Exon I, the same reported as "pre-resilin" [5, 11-14]), including 323 amino acids, and dominated by 18 copies of a 15 amino acid sequence "GGRPSDSYGAPGGGN", with seven glycine $(\mathrm{G})$, two prolines $(\mathrm{P})$ and one tyrosine $(\mathrm{Y})$; the second exon (Exon II), which is a short chitin binding domain with 62 amino acids; the third exon (Exon III), which includes 235 amino acids and is dominated by 11 copies of a 13 amino acid sequence "GYSGGRPGGQDLG", with five glycines (G), one proline $(\mathrm{P})$, and one tyrosine $(\mathrm{Y})$ [1-14].

To fully understand the nanostructures and structural transition of resilin proteins, a systematic characterization pathway was organized through TMDSC to target glass transition temperatures, FTIR to identify secondary structures, synchrotron Real-time X-ray to identify crystallization properties, and AFM to observe morphology. Figure 1 shows the surface morphology of the full length resilin samples observed by AFM. The height image and its corresponding phase image of full length resilin are shown. The images show that the untreated full length resilin protein had a tightly-packed nanoparticle pattern with a particle size around $25 \sim 50 \mathrm{~nm}$, and the top-bottom height about $2 \sim 5 \mathrm{~nm}$ on average.

In comparison, real-time wide angle X-ray scattering studies (WAXS) during the thermal heating were performed in the synchrotron X-ray beam line to identify the structures in full length resilin samples before and after their glass transitions. The experiments were performed at $2 \mathrm{~K} / \mathrm{min}$ heating from $30^{\circ} \mathrm{C}$ to $270^{\circ} \mathrm{C}$, and the temperature development of the wide angle $\mathrm{X}$-ray scattering pattern of the full length resilin was shown in Figure 2. The amorphous pattern is the first scan of the sequence. Different peaks appeared for the full length 
resilin protein samples during the real-time wide angle $\mathrm{X}$ ray study, which were directly related to their protein structures.

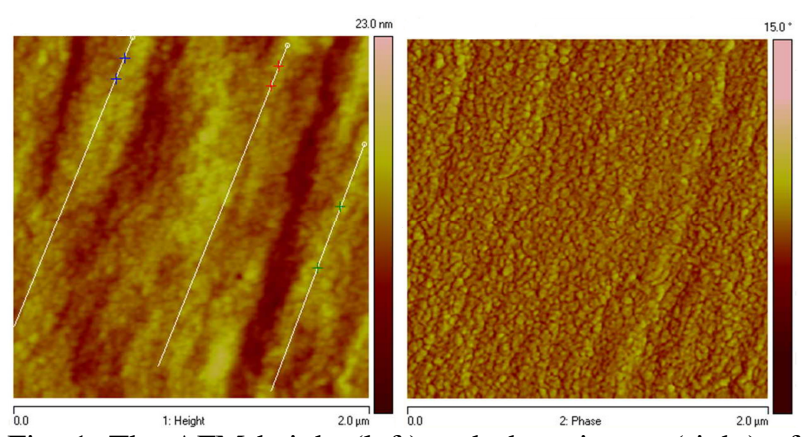

Fig. 1. The AFM height (left) and phase image (right) of the full length resilin protein films. The height of the particles was calculated using line analysis measurements (middle) through the line traces shown on the Figure on the left.

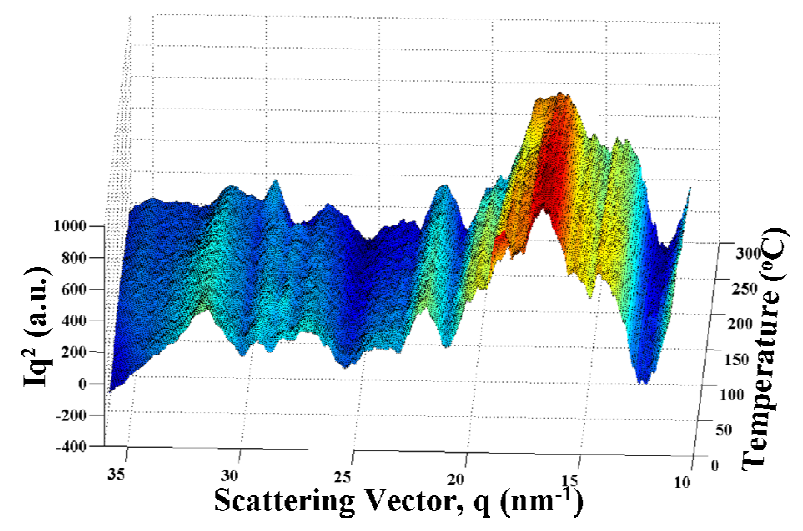

Fig. 2. Temperature developments in the wide angle $\mathrm{X}$ ray scattering pattern of the full length resilin protein.

\section{CONCLUSIONS}

The morphology and structure of full length resilin is discussed in this work. A thermal energy input method was used to investigate the transitions and structural changes of the resilin protein. A systematic pathway for studying this mechanism is organized by using TMDSC, real-time FTIR, synchrotron real-time X-ray, and AFM. The results of this approach provided structural information about these proteins which could be widely useful for the future applications as elastomeric biomaterials.

\section{ACKNOWLEDGEMENT}

The authors thank the NIH, the Air Force Office of Scientific Research and DARPA for support, and the MRI Program for thermal analysis instrumentation under DMR-0520655.

\section{REFERENCES}

[1] T. Weis-Fogh, "A rubber-like protein in insect cuticle," J. Exp. Biol., 1960, vol. 37, pp. 887-907.

[2] H. C. Bennet-Clark, E. C. A. Lucey, "The jump of the flea: a study of the energetics and a model of the mechanism," J. Exp. Biol., 1967, vol. 47 , pp. 59-67.

[3] D. Young, H. C. Bennet-Clark, "The role of the tymbal in cicada sound production," J. Exp. Biol., 1995, vol. 198, pp. 1001-1020.

[4] R. L. Lyons, E. Lesieur, M. Kim, D. C. C. Wong, M. G. Huson, K. M. Nairn, et. al., Protein Eng., Des. Sel., 2007, vol. 20, pp. 25-32.

[5] C. M. Elvin, A. G. Carr, M. G. Huson, J. M. Maxwell, R. D. Pearson, T. Vuocolo, et. al., "Synthesis and properties of crosslinked recombinant pro-resilin," Nature, 2005, vol. 437, pp. 999-1002.

[6] G. Qin, S. Lapidot, K. Numata, X. Hu, S. Meirovitch, M. Dekel, I. Podoler, O. Shoseyov, D. L. Kaplan, "Expression, Cross-Linking, and Characterization of Recombinant Chitin Binding Resilin," Biomacromolecules, 2009, vol. 10, pp. 3009-3014.

[7] D. H. Ardell, S. O. Andersen, "Tentative identification of a resilin gene in Drosophila melanogaster," Insect Biochem. Mol. Biol., 2001, vol. 31, pp. 965-970.

[8] M. G. Huson, J. M. Maxwell, "The measurement of resilience with a scanning probe microscope, " Polym. Test., 2006, vol. 25, pp.2-11.

[9] M. V. Karouzou, Y. Spyropoulos, V. A. Iconomidou, R. S. Cornman, S. J. Hamodrakas, J. H. Willis, "Drosophila cuticular proteins with the R\&R Consensus: annotation and classification with a new tool for discriminating RR-1 and RR-2 sequences," Insect Biochem. Mol. Biol., 2007, vol. 37, pp. 754-760.

[10] M. Kim, C. Elvin, A. Brownlee, R. Lyons, "High yield expression of recombinant pro-resilin: lactose-induced fermentation in E. coli and facile purification," Protein Expr. Purif., 2007, vol. 52, pp. 230-236. [11] K. M. Nairn, R. E. Lyons, R. J. Mulder, S. T. Mudie, D. J. Cookson, E. Lesieur, M. Kim, D. Lau, F. H. Scholes, C. M. Elvin, "A synthetic resilin is largely unstructured,” Biophys. J., 2008, vol.95, pp. 3358-3365. [12] M. B. Charati, J. L. Ifkovits, J. A. Burdick, J. G. Linhardt, K. L. Kiick, "Hydrophilic Elastomeric Biomaterials based on Resilin-like Polypeptides," Soft Matter, 2009, vol. 5, pp. 3412-3416.

[13] N. K. Dutta, N. R. Choudhury, M. Y. Truong, M. Kim, C. M.Elvin, A. J. Hill, "Physical approaches for fabrication of organized nanostructure of resilin-mimetic elastic protein rec1-resilin," Biomaterials, 2009, vol. 30, pp. 4868-4876.

[14] R. E. Lyons, K. M. Nairn, M. G. Huson, M. Kim, G. Dumsday, C. M. Elvin, "Comparisons of Recombinant Resilin-like Proteins: Repetitive Domains Are Sufficient to Confer Resilin-like Properties," Biomacromolecules, 2009, vol. 10, pp. 3009-3014. 


\title{
Effect of Aging and Degeneration on the Human Intervertebral Disc during the Diurnal Cycle: A Finite Element Study
}

\author{
${ }^{1}$ C.J. Massey, ${ }^{2}$ C.C. van Donkelaar, ${ }^{1} \mathrm{M}$ Marcolongo \\ ${ }^{1}$ Drexel University, Philadelphia, PA \\ ${ }^{2}$ Eindhoven University of Technology, Eindhoven, The Netherlands
}

\begin{abstract}
Alterations in the major biochemical constituents of intervertebral discs coincide with aging and degeneration, and can alter the disc's ability to support load. The most significant biochemical change that occurs in degeneration is the loss of proteoglycans in the nucleus pulposus. During a diurnal cycle, the disc experiences approximately 16 hours of functional loading, followed by 8 hours of recovery. An axisymmetric, poroelastic model was created using ABAQUS finite element software. Standard poroelastic theory is utilized, but a userdefined material was written to include the effects of osmotic swelling, which is directly related to proteoglycan content. Due to the high stresses in the nucleus, the annulus fibrosus must remodel itself to account for the change in properties of the nucleus. The stress experienced by the nucleus increases greatly in Grade 2 from Grade 1, but then decreases in Grade 3, and even Grade 4 experiences lower stresses than in Grade 2. The osmotic pressure in the central nucleus decreases approximately $75 \%$ with degeneration. This explains the increasing inability of Grades 3 through 5 to recover the fluid lost during loading, since the osmotic pressure gradient is the primary mechanism with which fluid flows back into the disc.
\end{abstract}

\section{INTRODUCTION}

Intervertebral disc (IVD) degeneration occurs with aging, and may be a major cause of back pain. The IVD is the primary compression-carrying component of the spine. Its roles are to transmit and distribute loads, and allow for the necessary flexibility of the spine. It is comprised of a central gel-like nucleus pulposus (NP), an outer annulus fibrosus (AF), and upper and lower endplates consisting of cartilaginous and bony portions. Alterations in the major biochemical constituents of the IVD have been shown to coincide with aging and disc degeneration and can subsequently alter the discs' ability to support load. The most significant biochemical change that takes place in disc degeneration is the loss of proteoglycans in the NP [1]. These hydrophilic molecules help to retain and replenish the fluid in the disc.

The role of fluid in the NP is vital to the IVD's ability to handle loads. As a healthy disc experiences normal daily loading, a swelling pressure is generated inside the NP, transferring the load to the AF. The ability of the NP to absorb and retain water is a function of its chemical composition, most notably its proteoglycan content [2].

During a diurnal cycle, the IVD experiences approximately 16 hours of functional loading (standing, sitting, etc.), followed by 8 hours of recovery (lying prone). Therefore, the fluid lost during the functional loading period must be replenished in half the time. As the disc is compressed and fluid is exuded, the density of the fixed charges within the nucleus pulposus is increased, creating an osmotic gradient with the interstitial fluids surrounding the disc. This osmotic potential aids in drawing fluid back into the disc.

\section{METHODS}

An axisymmetric, poroelastic model was created using ABAQUS v6.5 finite element software. The model consists of an NP, an AF, cartilaginous and bony portions of the adjacent endplates, and cancellous and cortical portions of the corresponding vertebrae. The standard poroelastic theory is utilized, but a user-defined material was written to include the effects of osmotic swelling. The model response was validated against experimental results such as axial displacement, radial displacement of the outer AF, and total fluid lost.

Material properties for each tissue were referenced from literature. However, where these properties were not reported for each degenerative grade, values at each end of the spectrum were gathered and the intermediate values were interpolated. Where no specific degenerative results existed, assumptions were made based on the effects of degeneration on the tissue.

Fixed charge density profiles for healthy (Grade 1) and degenerated (Grade 5) are shown in Fig. 1, as adapted from

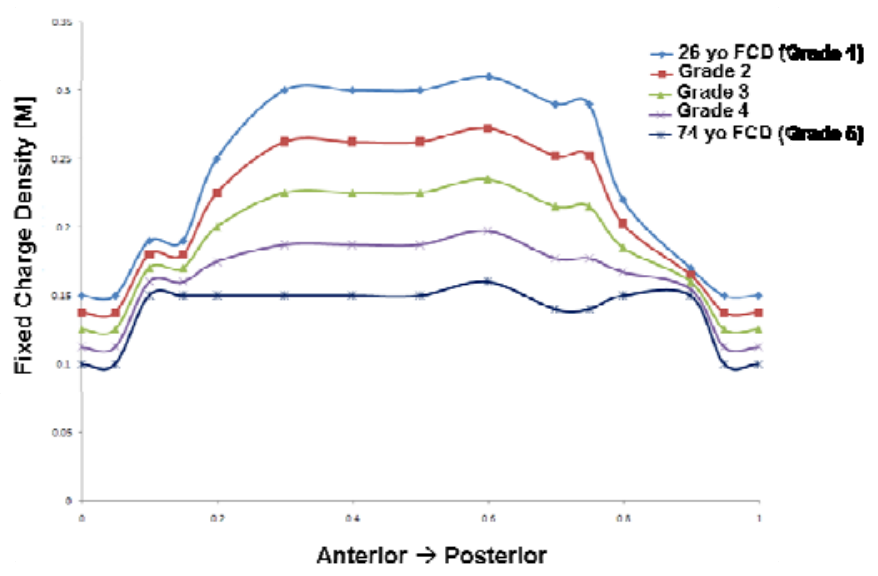

Fig. 1. The interpolated fixed charge density profiles used to model changes in PG content of the IVD with degeneration 
Urban [3]. Although the 26 year old disc may not be a Grade 1 , it is treated as such for the purpose of this study, as is the 74 year old as a Grade 5. The profiles for Grades 2-4 were linearly interpolated from these reported values.

The unit was loaded with a 0.5 MPa pressure on the upper vertebra, and a $0.1 \mathrm{MPa}$ recovery load. As is seen in experimental studies, a steady-state condition is found after several loading and recovery cycles due to the exchange of fluid. Therefore, each simulation consisted of 4 diurnal cycles, with the last one being considered the steady-state cycle.

\section{RESULTS}

Fig. 2 shows the von Mises stress contour plots of the NP and AF, as well as the NP by itself. This stress value is the stress experienced by the tissue, which is found by taking the stress in the solid portion of the tissue minus the osmotic pressure. Stress at the NP-AF interface increases with degeneration, as does the stress in the majority of the AF. There is an increase in the center of the NP, as well as the NP side of the NP-AF interface.

Pore pressure in the central NP can be seen to increase with degeneration. The high pore pressures are not just contained in the NP, but spill over the NP-AF interface into the inner AF. The outer AF has practically no pore pressure due to the free-flow boundary condition there. The steady state pore pressure does not change very much with degeneration.

For each grade, the highest osmotic pressures are seen in the central NP, and decrease radially outwards towards the outer AF. The central NP decreases the most with degeneration.

\section{DISCUSSION}

The AF experiences the highest stress at its interface with the NP and at the outer corners, where the attachment to the

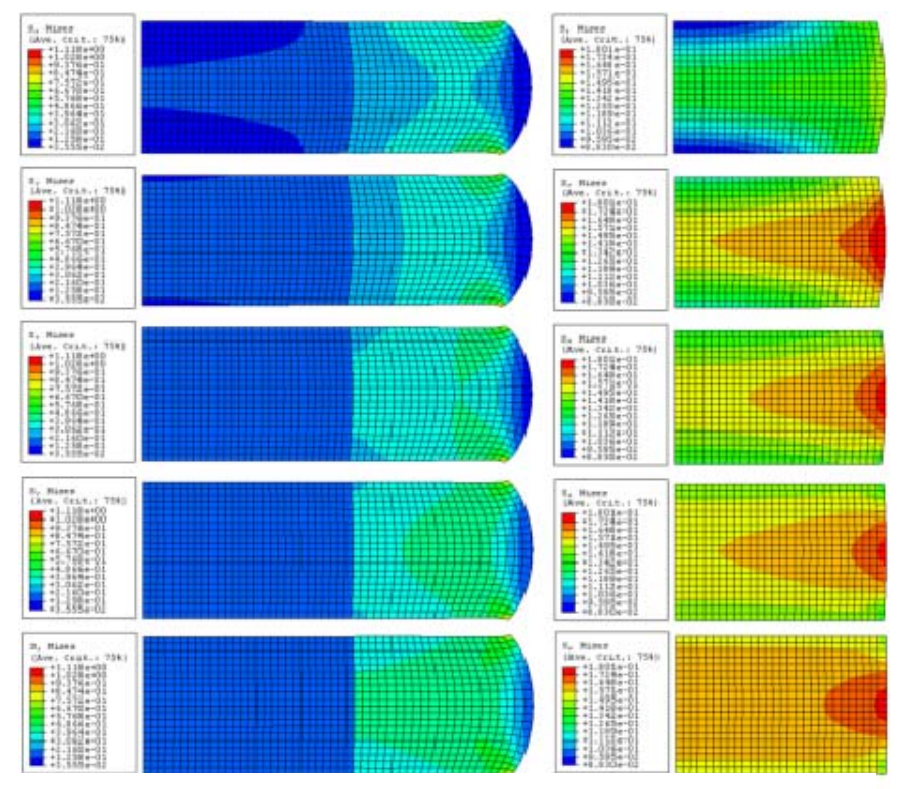

Fig. 2. Comparison of the von Mises stress profiles for Grades 1 through 5 of the NP and AF (left) and NP only (right) cartilaginous endplates causes a high stress concentration. Both of these are artifacts of the model. The high concentration of stress at the NP-AF interface is likely an artifact of the abrupt change in material properties across the interface. In the actual tissue, there is a transition zone, which would prevent this by gradually changing properties. Also, in the actual tissue, the AF connects directly to the adjacent vertebrae around the outer edge, and the endplates are completely covered by the annulus fibrosus. There are also no sharp angles or edges in the actual tissue, which is a major cause of stress concentrations.

Due to the high stresses in the NP, the AF must remodel itself to account for the change in properties of the NP. The stress experienced by the NP increases greatly in Grade 2 from Grade 1, but then decreases in Grade 3, and even Grade 4 experiences lower stresses than in Grade 2.

The osmotic pressure in the central NP decreases approximately $75 \%$. This explains the increasing inability of Grades 3 through 5 to recover the fluid lost during the loading periods, since the osmotic pressure gradient is the primary mechanism with which fluid flows back into the disc.

For the steady-state cycle, pore pressure does not change with degeneration. However, pore pressure after the first hour of the first cycle is the smallest at the outer AF, since this boundary allows free exchange of fluid across it. Moving radially inwards towards the center of the NP, the pore pressure increases at every grade. The entire NP and inner AF have the highest values. The pore pressure increases as degeneration occurs, likely due to the decrease in the permeability of the bony endplate from Grade 1 to Grade 5 . Since this is the smallest permeability value, it begins the rate limiter of fluid leaving the disc through the endplates.

\section{REFERENCES}

[1] Raj, P. and A. FIPP, Intervertebral Disc: Anatomy-PhysiologyPathophysiology-Treatment. Pain Practice, 2008. 8(1): p. 18.

[2] Bao, Q. and H. Yuan, Artificial Disc Technology. Neurosurgical Focus, 2000. 9(4): p. 1-9.

[3] Urban, J.P.G. and S.H. Holm, Intervertebral Disc Nutrition as Related to Spinal Movements and Fusion, in Tissue Nutrition and Viability, H. AR, Editor. 1986, Springer-Verlag: New York. p. 101-119. 


\title{
Engineering Three-dimensional Nervous Tissue Constructs Based on Fiber-Gel Substrates
}

\author{
M. L. Siriwardane and B. J. Pfister \\ Department of Biomedical Engineering, New Jersey Institute of Technology, Newark, NJ 07102
}

\begin{abstract}
Three-dimensional (3D) nervous tissue constructs were developed using a compliant gel matrix and a fiber component for promoting contact-guided axon outgrowth. Rat embryonic (E15) dorsal root ganglion explants (DRGs) were cultured on biodegradable poly-L-lactic acid (PLLA) and wet-spun type I collagen fibers embedded in soft collagen gels. Gel constructs containing fibers induced greater axon outgrowth distances with significantly more directionality along the aligned fibers. Interestingly, wetspun type I collagen from rat tail tendon displayed a highly aligned outgrowth pattern from DRG axons suggesting potential use for peripheral nerve repair.
\end{abstract}

\section{INTRODUCTION}

Biomolecular cues are well known to promote specificity in synapse formation and axon guidance to appropriate targets, however, much less is known about physical cues during regeneration [1]. Mechanical cues presented by 2D rigid substrates induce far greater neurite extension than cells cultured in 3D substrates [2]. Axon growth preference for rigid substrates may have been explained many years ago by Lamoureux, Heidemann, and several others who discovered that growth rates of neurites were strongly correlated to applied tension, in which a pulling phenomenon was observed during elongation of growth cone $[3,4]$. Although growing axons favor the $2 \mathrm{D}$ substrates, high demand for developing 3D substrates/gels/scaffolds to provide a more "biomimetic" environment still remains. Interestingly, earlier work conducted by Gomez and Letourneau demonstrated that when presented a choice, growth cones follow the preferred of 2 substrates [5]. Previous work in the area of developing 3D constructs have led to a strong belief that ideal constructs may involve incorporating "2D-like" substrates/surfaces in 3D space [2].

Wet spinning is a practical approach for creating biomimetic scaffolds from natural polymers like collagen while retaining biological characteristics $[6,7]$. Collagen fibrils from rat tail tendon, which have rarely been explored for neural applications, may present physical cues ideal for contact guidance of axons. Interestingly, this study investigated whether DRG axons in the presence of fibers within a soft hydrogel would elicit a preferential contact guidance response.

\section{A. Materials}

\section{MATERIALS AND METHODS}

PLLA fibers $(50-200 \mu \mathrm{m})$ used in this study were provided by Dr. Zohar Ophir, Medical Device Concept Laboratory, New Jersey Institute of Technology. Type I collagen used for gel matrices and for wet spinning fibers was obtained via extraction [8] from rat tail tendons of euthanized SpragueDawley rats $(200-250 \mathrm{~g})$ at the Rutgers-Newark Animal Facility. DRG neurons were isolated from E15 rat embryos using a previously established protocol [9].

\section{B. Wet spinning}

Type I collagen in lyophilized form following extraction was reconstituted in glacial acetic acid to obtain a $0.75 \mathrm{wt} \%$ collagen dispersion at $\mathrm{pH} 4$, which was loaded into a $10 \mathrm{cc}$ glass syringe. The syringe was mounted onto a syringe pump (Harvard Systems), with Viton tubing connecting the syringe to a blunt-tipped, 22-gauge needle (spinneret). The tip of the spinneret was immersed into a coagulation bath containing HPLC-grade acetone and ammonium hydroxide at $\mathrm{pH}$ 9. The syringe pump was adjusted to a constant flow rate of $0.0136 \mathrm{~mL} / \mathrm{min}$. As the dispersion was extruded into the coagulation bath, a firm collagen fiber began to form and was collected from the bath.

\section{Hydrogel preparation}

Type I collagen gels from rat tail tendon were prepared at approximately $0.8 \mathrm{mg} / \mathrm{mL}$ concentration using the following mixture: $10 \mu \mathrm{L}$ of $1 \mathrm{~N} \mathrm{NaOH}, 180 \mu \mathrm{L}$ of tissue culture grade $\mathrm{H}_{2} \mathrm{O}, 800 \mu \mathrm{L}$ of rat type I collagen $(\sim 4.0 \mathrm{mg} / \mathrm{mL})$, and 100 $\mu \mathrm{L}$ of 10X MEM (Sigma), $59.58 \mathrm{~g} / \mathrm{L}$ HEPES (Sigma), and $22.0 \mathrm{~g} / \mathrm{L} \mathrm{NaCHO}_{3}$ (Sigma). Approximately, 300 $\mu \mathrm{L}$ of $0.8 \mathrm{mg} / \mathrm{mL}$ collagen gel was filled into 12 -well plate. Single PLLA and collagen filaments were then load onto the gel layer and incubated up to $1 \mathrm{hr}$ prior to cell plating (Fig. 1).

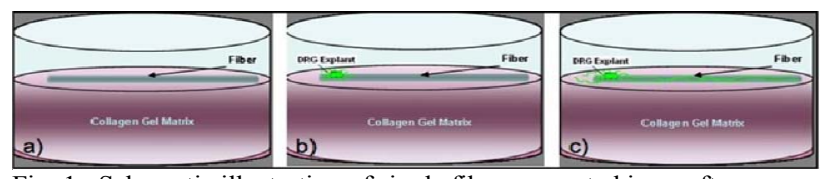

Fig. 1. Schematic illustration of single fiber supported in a soft, $0.8 \mathrm{mg} / \mathrm{ml}$ collagen gel using a primary rat DRG explant.

\section{RESULTS}

Neurite lengths were determined by using composite images of DRG explants stained with Calcein AM (Invitrogen) at 2, 5, 8, and 10 days in vitro (DIV). Images were merged to create montages using Photoshop (Adobe). Lengths of neurites were measured from the surface of the DRG explant to their farthest tips using Image $\mathrm{J}$ software (NIH). 
Rat DRG explants were used to assess whether the wetspun collagen micron fibers could act as suitable substrates for axon outgrowth. Synthetic PLLA fibers of similar fiber diameter were compared with collagen fibers wet-spun from bovine tendon and rat tail tendon in this study. Neurite outgrowth was observed after $24 \mathrm{hr}$ of incubation. The direction of neurite extension exhibited varying degrees of orientation depending on the fiber group (Fig. 2). Although fiber orientation was mostly parallel to the aligned monofilament in all fiber groups, unmodified PLLA fibers influenced neurite alignment much less with mean outgrowth distance of $2.93 \pm 0.17 \mathrm{~mm}$ (Fig. 3). PLLA fibers coated with rat tail collagen showed significant number of neurites oriented parallel to the fiber axis with greatly enhanced mean outgrowth distance of $5.94 \pm 0.28 \mathrm{~mm}$. Interestingly, DRG neurites responded differently to bovine tendon and rat tail collagen fibers with mean outgrowth distances of $3.52 \pm 0.17$ $\mathrm{mm}$ and $5.65 \pm 0.26 \mathrm{~mm}$, respectively.

A control group consisting only of gel constructs revealed minimal neurite extension of approximately $1.28 \pm 0.27 \mathrm{~mm}$. Furthermore, 2D control groups of $35 \mathrm{~mm}$ dishes coated with collagen were used to quantify neurite outgrowth and to compare with $3 \mathrm{D}$ neurite outgrowth data. Average neurite extension in 2D was approximately $2.60 \pm 0.54 \mathrm{~mm}$ (Fig. 4).
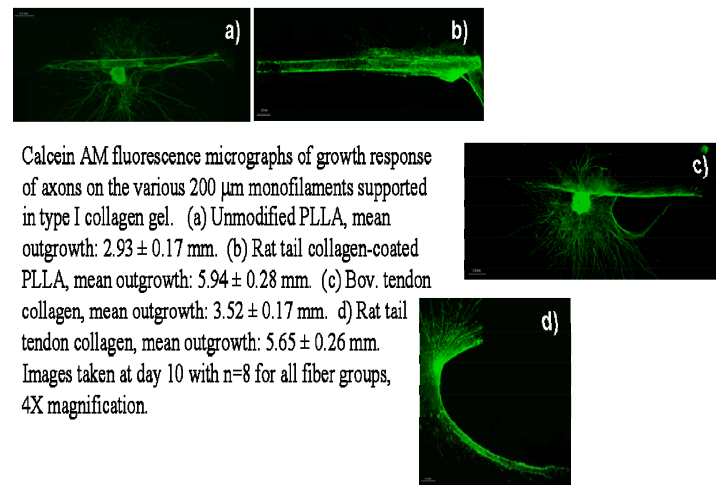

Fig. 2. Fluorescence images of DRG axon outgrowth on different fiber groups at day 10 of culture.

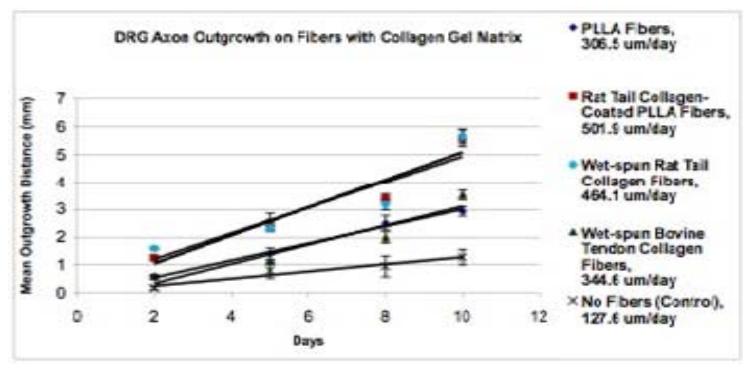

Fig. 3. Mean axon outgrowth distances and rates up to 10 DIV.

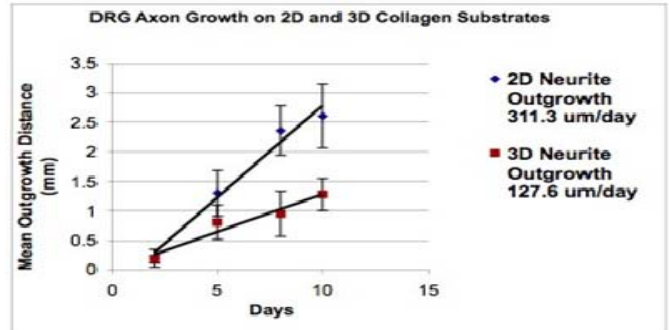

Fig. 4. Comparison of DRG axon growth on 2D and 3D collagen substrates.

\section{DISCUSSION}

Neurite outgrowth results validated that the incorporation of fibers into 3D gels strongly influenced contact-guided axon growth by presenting growth cones of axons with choices in 3D space. Soft, confluent collagen gels were mechanically stable and proved to be growth permissive for axons, which correlates with previous studies [10]. The lowest neurite extension distances on unmodified PLLA fibers implies that distributing fibers in 3D gels yielded comparable neurite outgrowth distances to $2 \mathrm{D}$ controls. Interestingly, neurite extension lengths of fiber-gel constructs significantly surpassed 2D control groups when fibers contained some collagen component on its surface.

\section{ACKNOWLEDGEMENT}

This work was supported by the New Jersey Commission on Brain Injury Research 07-3204-BIR-E-0, NSF CAREER CBET-0747615, and NSF C ${ }^{2}$ PRISM-0638423.

\section{REFERENCES}

Q. T. Nguyen, Sanes, J. R., Lichtman, J. W., "Pre-existing pathways promote precise projection patterns," Nature Neuroscience, vol. 5, no. 9, pp. 861-867, 2002.

R. V. Bellamkonda, "Peripheral nerve regeneration: An opinion on channels, scaffolds and anisotropy," Biomaterials, vol. 27, pp. 35153518, 2006.

P. Lamoureux, Zheng, J., Buxbaum, R.E., and Heidemann, S.E., "A cytomechanical investigation of neurite growth on different culture surfaces," Journal of Cell Biology, vol. 118, pp. 655-661, 1992.

J. Zheng, Lamoureux, P., Santiago, V., Dennerll, T., Buxbaum, R.E., and Heidemann, S.R., "Tensile regulation of axonal elongation and initiation," Journal of Neuroscience, vol. 11, pp. 1117-1125, 1991 T. M. Gomez, and Letourneau, P.C., "Filopodia initiate choices made by sensory neuron growth cones at laminin/fibronectin borders in vitro," J Neurosci, vol. 14, no. 10, pp. 5959-5972, 1994.

B. B. Crow, Borneman, A.F., Hawkins, D.L., Smith, G.M., and Nelson, K.D., "Evaluation of in Vitro Drug Release, $\mathrm{pH}$ Change, and Molecular Weight Degradation of Poly(L-lactic acid) and Poly(D,L-lactide-coglycolide) Fibers," Tissue Engineering, vol. 11, no. 7/8, pp. 1077-1084, 2005.

D. e. a. Zeugolis, "Electro-spinning of pure collagen nano-fibres e Just an expensive way to make gelatin?," Biomaterials, vol. 29, pp. 2293$2305,2008$.

[8] N. Rajan, Habermehl, J., Cote', M., Doillon, C.J., and Mantovani, D., "Preparation of ready-to-use, storable and reconstituted type I collagen from rat tail tendon for tissue engineering applications," Nature Protocols, vol. 1, no. 6, pp. 2753-2758, 2007.

[9] N. Kleitman, Wood, P.M., and Bunge, R.P., "Tissue culture methods for the study of myelination," 1998.

[10] R. K. Willits, Skornia, S.L. , "Effect of collagen gel stiffness on neurite extension.," J. Biomater. Sci. Polymer Edn, vol. 15, no. 12, pp. 1521-1531, 2004. 


\title{
Nanofiber Alignment Regulates Adhesion and Integrin Expression of Human Mesenchymal Stem Cells and Tendon Fibroblasts
}

\author{
${ }^{1}$ S.P. Kwei, ${ }^{1}$ K.L. Moffat, ${ }^{2}$ W. N. Levine, and ${ }^{1}$ H. H. Lu \\ ${ }^{1}$ Department of Biomedical Engineering, Columbia University, ${ }^{2}$ Orthopaedic Surgery, Columbia University, USA
}

\begin{abstract}
Rotator cuff tears are among the most common shoulder injuries that require surgery. High failure rates of biological graft-based repairs underscore the need for functional alternatives. In this study, effects of nanofiber organization on adhesion of cuff fibroblasts (hRCF) and human mesenchymal stem cells (hMSC) are evaluated. It is hypothesized that fiber alignment will regulate cell morphology and integrin gene expression. hRCF and hMSC were seeded on aligned and unaligned nanofiber scaffolds of polylactide-co-glycolide. Cell morphology $(n=3)$ and gene expression $(n=5)$ for integrins $\alpha 2$, $\alpha V, \alpha 5$, and $\beta 1$ were determined over 14 days. Cell morphology was found to differ between groups. Higher $\alpha 2$ and $\beta 1$ expressions in hRCF and hMSC on aligned scaffolds suggest aligned scaffolds a more biomimetic structure to native tendon, since integrin $\alpha 2 \beta 1$ facilitates cell attachment to collagenous matrices. Expressions for $\alpha \mathrm{V}$ and $\alpha 5$, which are associated with tendon healing, were significantly higher on unaligned scaffolds, and suggest a healing response by the cells. In conclusion, the cells may recognize differences in matrix organization, and fiber alignment regulates cell adhesion. Moreover, the aligned nanofiber matrix may promote a more biomimetic fibroblast response than the unaligned scaffold.
\end{abstract}

\section{INTRODUCTION}

Rotator cuff tendon repair is one of the most common shoulder procedures performed annually in the United States[1]. Failure rates for primary cuff repair have been reported to be as high as $94 \%[2]$, and the use of biological grafts has yielded suboptimal results[3]. To improve tendon repair, we have developed a degradable polymer nanofiberbased scaffold system[4], and are currently evaluating the response of human mesenchymal stem cells (hMSC) on this novel scaffold. As an in-depth understanding of the mechanism of cell-material interactions will be essential for the design and optimization of these biomimetic scaffolds, the objective of this study is to compare the adhesion of hMSC and human rotator cuff fibroblasts (hRCF) as a function of nanofiber alignment. It is hypothesized that nanofiber organization will regulate cell adhesion morphology and integrin expression. Moreover, distinct responses are expected between the undifferentiated and differentiated cell types.

\section{MATERIALS AND METHODS}

\section{Scaffold Fabrication and Characterization}

Aligned and unaligned nanofiber scaffolds of poly(lacticco-glycolic) acid (PLGA 85:15, Lakeshore) were produced by electrospinning[4]. Scaffold fiber organization was examined with Scanning Electron Microscopy (SEM, n=3) and confirmed by quantitative alignment analysis[5].

\section{Cells and Cell Culture}

Explant-derived human rotator cuff tendon fibroblasts (hRCF, 49-72 y/o, passage $=6$ ) and hMSC (Cambrex, passage =9) were seeded on the nanofiber scaffolds at a density of $3.14 \times 10^{4}$ cells $/ \mathrm{cm}^{2}$ and maintained in fully supplemented medium containing $10 \%$ fetal bovine serum (FBS), $1 \%$ antibiotics, $1 \%$ non-essential amino acids, and $0.1 \%$ antifungal.

\section{End-point Analyses (1,3 \& 14 days)}

Cell adhesion and viability was assessed by Live/Dead assay $(n=2)$ with confocal microscopy on days 1 and 14 . Gene expression $(n=5)$ for integrins $\alpha 2, \alpha \mathrm{V}, \alpha 5, \beta 1$, and GAPDH was evaluated by RT-PCR.

\section{RESULTS}

The adhesion of both hMSC and fibroblasts was guided by nanofiber alignment, and distinct cell morphologies were observed on the two types of substrates (Fig. 1).

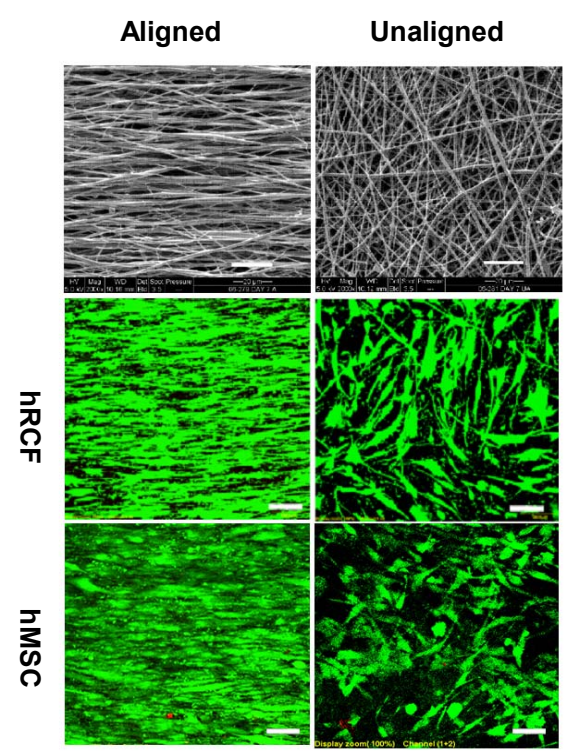

Figure 1: SEM of aligned and unaligned scaffolds (top panel, 2000x, bar $=20 \mu \mathrm{m}$ ). Fibroblast (middle panel) and stem cell (bottom panel) viability and morphology on days 1 and $14(20 x, b a r=100 \mu m)$. 
Distinct integrin gene expression profiles were observed on unaligned and aligned nanofiber scaffolds, with significant differences detected between hMSC and fibroblasts (Figs. 2A, 2B). Significantly higher expressions of $\alpha 2$ and $\beta 1$ (Fig 2A) were detected for fibroblasts on aligned nanofibers. In addition, $\alpha 2$ expression in hMSC is upregulated over time on the aligned scaffolds (Fig 2B). Both $\alpha \mathrm{V}$ and $\alpha 5$ are upregulated by day 14 on unaligned scaffolds in both cell types.

A
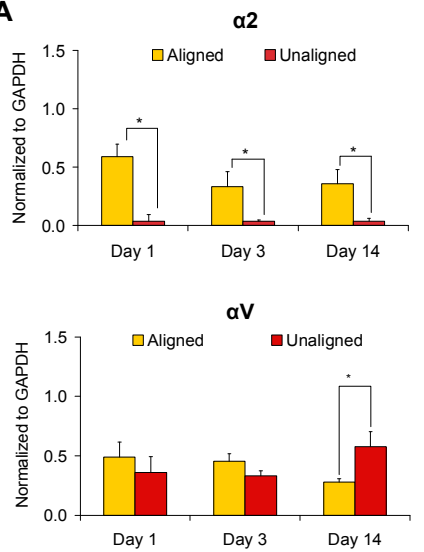

a5

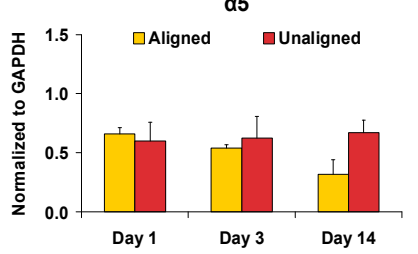

$\beta 1$

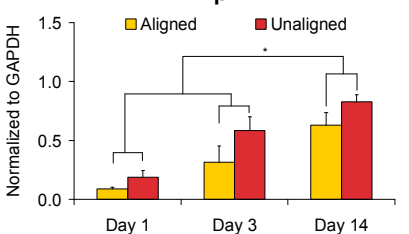

Figure 2: Normalized gene expression for integrins $\alpha 2, \alpha \mathrm{V}, \alpha 5, \beta 1$ on unaligned and aligned scaffolds seeded with $\mathbf{A})$ hRCFs and B) hMSCs, $\left({ }^{*} \mathrm{p}<0.05\right)$.

\section{DISCUSSION}

The alignment of hMSC and fibroblasts on the aligned and unaligned nanofiber scaffolds was guided by nanofiber alignment. Specifically, both cell types attached along the fiber axis on aligned scaffolds, while the cells showed no preferred orientation on the unaligned scaffolds. In conjunction with distinct cell morphologies observed on the two substrates, it is suggested that the cells recognize differences in fiber organization.

For gene expression, significantly higher expressions of $\alpha 2$ and $\beta 1$ were observed for fibroblasts seeded on aligned scaffolds. As these two integrins form a heterodimer that mediates cell attachment to collagen[6], their upregulation on aligned nanofibers suggests that this substrate may better mimic the native tendon matrix.
Interestingly, upregulation of $\alpha 2$ was observed in hMSC seeded on aligned scaffolds, indicating that matrix alignment may promote hMSC differentiation into tendon fibroblasts. Finally, as both $\alpha \mathrm{V}$ and $\alpha 5$ have been associated with tendon healing[7], their upregulation by day 14 on unaligned scaffolds in both cell types may be more indicative of a repair response, which may result in formation of unorganized matrix or callus. It is noted that the scaffolds in this study exhibit similar structural properties (nanofiber diameter, porosity, pore size, permeability)[4]; thus any observed differences in cell adhesion can be attributed largely to nanofiber organization (aligned vs. unaligned). In summary, nanofiber alignment guides hMSC and fibroblast adhesion, with the aligned nanofiber matrix inducing a more biomimetic response. Future work will focus on determining integrin production and the downstream effects of these observed cell responses as a function of nanofiber organization.

\section{ACKNOWLEDGEMENT}

The study was supported by the National Institute of Health, National Institute of Arthritis and Musculoskeletal and Skin Diseases (NIH/NIAMS R21-AR056459, R01AR055280).

\section{REFERENCES}

[1] Vitale, M. A., Vitale, M. G., Zivin, J. G., Braman, J. P., Bigliani, L. U. \& Flatow, E. L. Rotator cuff repair: an analysis of utility scores and cost-effectiveness. J Shoulder. Elbow. Surg 16, 1812007.

[2] Galatz, L. M., Ball, C. M., Teefey, S. A., Middleton, W. D. \& Yamaguchi, K. The outcome and repair integrity of completely arthroscopically repaired large and massive rotator cuff

tears. J. Bone Joint Surg. Am. 86-A, 2192004.

[3] Sclamberg, S. G., Tibone, J. E., Itamura, J. M. \& Kasraeian, S. Six-month magnetic resonance imaging followup of large and massive rotator cuff repairs reinforced with porcine small intestinal submucosa. J. Shoulder Elbow Surg. 13, 5382004.

[4] Moffat, K. L., Kwei, S. P., Spalazzi J. P., Doty, S., Levine, W., Lu, H. H. Novel Nanofiber-Based Scaffold for Rotator Cuff Repair and Augmentation. Tissue Eng 15. 115 2009.

[5] Costa, K. D., Lee, E. J. \& Holmes, J. W. Creating alignment and anisotropy in engineered heart tissue: role of boundary conditions in a model three-dimensional culture system. Tissue Eng 9, 5672003.

[6] Gallant, N.D. and García, A.J. Model of integrin-mediated cell adhesion strengthening. J. Biomechanics 40, 13012007.

[7] Harwood, F. L., Monosov, A. Z., Goomer, R. S., Gelberman, R. H., Winters, S. C., Silva, M. J. \& Amiel, D. Integrin expression is upregulated during early healing in a canine intrasynovial flexor tendon repair and controlled passive motion model. Connect. Tissue Res 39, 3091998. 


\title{
Patterning Micro-stiffness in Cell-Adhesive Substrate Using Microfluidics-based Lithography
}

\author{
Yuk Kee Cheung ${ }^{1}$, Evren U. Azeloglu ${ }^{1}$, David Shiovitz ${ }^{1}$, Kevin D. Costa ${ }^{1}$, Dror Seliktar ${ }^{2}$, and Samuel K. Sia ${ }^{1}$ \\ ${ }^{1}$ Department of Biomedical Engineering, Columbia University, 351 Engineering Terrace, 1210 Amsterdam Avenue, New York, NY 10027, USA \\ ${ }^{2}$ Biomedical Engineering Department, Technion-Israel Institute of Technology, Technion City, Haifa 32000, Israel
}

\begin{abstract}
In this study, we demonstrate the formation of 3D cell-adhesive hydrogels exhibiting well-defined spatial variation in stiffness using our previously developed microfluidics-based lithography technique [12]. PEG monoacrylate-linked bovine fibrinogen (PEG-fibrinogen) is photopolymerized into specific, user-defined shapes inside a microchannel, and successive cycles of fabrication result in a heterogeneous structure with controlled regional variations in stiffness. Atomic force microscope (AFM) indentations were used to directly confirm the micro-stiffness distribution, and morphological and migratory behavior of cells in microenvironments of controlled variations in stiffness was characterized. Our approach allows control of microscale variations of stiffness in cell-adhesive substrates with high precision and flexibility and offers the opportunity to examine differential cell-ECM interactions relevant to a multitude of fundamental cellular processes [14].
\end{abstract}

\section{INTRODUCTION}

Heterogeneous variation of stiffness of cellular microenvironments in native tissues directs cellular processes such as spreading [1,2], migration [3], growth [4], and differentiation [5]. In particular, microscale variations in stiffness have been observed from subcellular $(<5 \mu \mathrm{m})$ to multicellular $(>300 \mu \mathrm{m})$ dimensions in healthy and diseased tissues of the brain [6], bone [7], heart [8], and cartilage [9]. To study the effect of stiffness heterogeneity in vitro, previous approaches have used collagen-coated polyacrylamide gels to construct substrates with single-step [3] or continuous [10] stiffness gradients. Most notably, these studies demonstrated preferential cell migration toward stiffer regions of substrate, a phenomenon known as durotaxis. In order to recreate the complexities of native tissues for in vitro studies, it is important to develop a technology for constructing celladhesive substrates that exhibit flexible spatial architecture with controllable local variations in stiffness that are not limited to monotonic or single-step stiffness changes.

\section{METHODS}

Fabricating stiffness patterns using microfluidic lithography

Precursor solutions with fixed amount of PEG-fibrinogen [11] ( 2 or $4 \mathrm{mg} / \mathrm{mL})$ and variable amounts of PEGDA (MW: 400 or 4000; Polysciences, Warrington, PA) were prepared in sterile PBS. Varying PEGDA concentrations produced different crosslinking densities and hence different stiffness in the resultant hydrogels. Photoinitiators and co-initiators were added to all precursors to facilitate photocrosslinking reaction during exposure to UV or green light. Confocal laser scanning microscope was used to demonstrate scanning of an area predefined in the microscope software and an epifluorescence microscope used a field diaphragm to define the shape and size of the exposure. Precursor solution was introduced into a

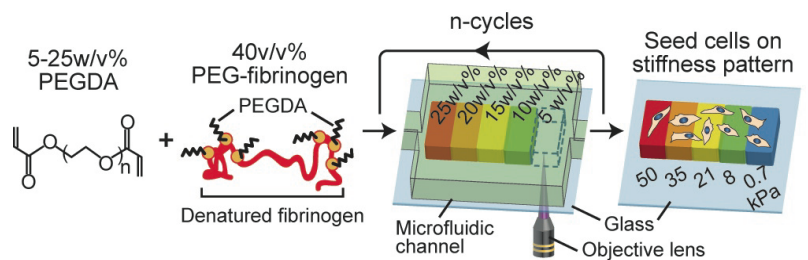

Figure 1. A schematic diagram of the experimental setup for using microfluidicsbased lithography to micropattern cell-adhesive substrates with defined heterogeneities in stiffness. Precursor solutions were prepared by adding various $\mathrm{w} / \mathrm{v} \%$ of PEGDA to a fixed amount of PEG-fibrinogen. During each cycle, a precursor solution was drawn into a microfluidic channel where it was photocrosslinked into hydrogel blocks.

microfluidic channel (similar to the one shown in Fig.1) and the exposure light was focused within the channel and photocrosslinked the hydrogel into the predefined shapes.

\section{Measurement of stiffness using AFM}

We verified the discrete stiffness gradient of our hydrogel patterns using AFM indentations, and roughness and topography information with AFM imaging. Apparent stiffness of each indentation force curve at an arbitrary indentation depth of $3 \mu \mathrm{m}$ was averaged for each array of probed points to obtain a mean stiffness value for the given PEGDA concentration.

\section{Image analysis for projected cell area and cell migration}

Phase-contrast images of plated human foreskin fibroblasts (HFFs) on different regions of a stiffness pattern were processed and analyzed using ImageJ (National Institutes of Health). To measure projected cell area, we manually segmented individual cells and measured the segmented area using ImageJ. One-way ANOVA tests determined statistical significance of differences between more than two groups of samples. For cell migration data, time lapse images of cells either in contact with stiffness boundaries or seeded on hydrogel of uniform stiffness were tracked for centroid positions using ImageJ and results were anaylyzed in MATLAB (MathWorks, Natick, MA).

\section{RESULTS AND DISCUSSION}

For an epifluorescence exposure system, the size and shape of the cell-adhesive microstructure patterned during each cycle are restricted by the power of the objectives and the geometry of the field diaphragm [13]. To achieve the micropatterning of discrete stiffness gradients containing multiple adjoining hydrogel blocks (Fig. 2a), we overlapped the exposure window onto portions of the microstructures formed in previous cycles. We also used a confocal laser scanning microscope to pattern PEG-fibrinogen with discrete variations 


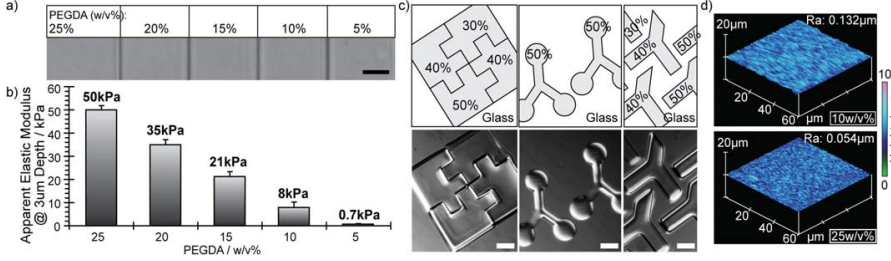

Figure 2. Fabrication of cell-adhesive substrate with discrete heterogeneities, and verification of stiffness using AFM. (a) discrete stiffness gradient. Scale bar is $50 \mu \mathrm{m}$. (b) Corresponding apparent elastic moduli of each section (mean $\pm \mathrm{SD}, \mathrm{n}=32$ ). (c) Fabrication of freeform patterns with components of varying stiffnesses using confocal laser scanning microscope. Scale bars are $100 \mu \mathrm{m}$. (d) Contact mode surface scans of hydrogels containing $10 \mathrm{w} / \mathrm{v} \%$ and $25 \mathrm{w} / \mathrm{v} \%$ of PEGDA and their respective average surface roughness $\left(R_{a}\right)$.

of stiffness, and demonstrated the formation of adjoining and free-standing hydrogel constructs of linear and curved geometries, and spanning a variety of scales (Fig. 2c). Use of the scanning laser allowed spatial control of stiffness patterns of $13 \mu \mathrm{m}$ or less (data not shown).

AFM results confirmed a discrete stiffness gradient that ranged from 50 to $0.7 \mathrm{kPa}$ (Fig. 2b) over a distance of $660 \mu \mathrm{m}$, with a constant step size of $130 \pm 1 \mu \mathrm{m}$ (Fig. 2a). We also observed minimal stiffness variation within each section of the discrete gradient, as intended (mean standard deviation was $2.3 \pm 2.3 \mathrm{kPa}$ ). Given that cell adhesion and migration can be influenced by the topography of the adhering substrate, we performed contact mode AFM imaging to characterize the surface topography and roughness of the discrete stiffness gradient. We observed an average surface roughness of $85.5 \mathrm{~nm}$ among hydrogels with 10-25w/v\% PEGDA (Fig. 2d).

Morphologically, HFFs on a discrete stiffness gradient spread along the gradient and assumed a characteristic spindle-like morphology by 10 hours after seeding (Fig. 3a). We observed a steady increase in projected cell areas with increasing gel stiffness after 10 hours of seeding (Fig. 3b). This finding is consistent with previous studies where cell area increases with gel stiffness in stiffness range of 1 to $40 \mathrm{kPa}[1$, $3,5,10]$. Over 22 hour time lapse of HFFs on cell-adhesive matrices with discrete stiffness gradient, $85 \%$ of the cells that were initially in contact with a boundary preferentially

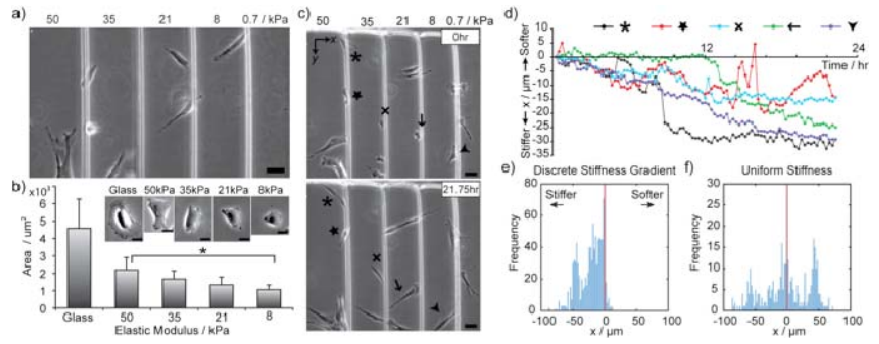

Figure 3. Morphological response and cell migration on discrete stiffness gradients. (a) HFFs plated on a discrete stiffness gradient (stiffness indicated by AFM reading). Scale bar is $50 \mu \mathrm{m}$. (b) Projected area of cells on separate hydrogels with stiffness ranges from 8 to $50 \mathrm{kPa}$ and glass at 10 hours after plating (mean \pm SEM, $\mathrm{n}=10-17$ ). The differences in average projected area among the four groups $(50,35,21,8 \mathrm{kPa})$ were statistically significant, as determined by ANOVA $\left({ }^{*} \mathrm{p}<0.05\right)$. Scale bars are $25 \mu \mathrm{m}$. (c) HFFs seeded onto a discrete stiffness gradient for 10 hours, followed by 22 hour time lapse. Scale bars are $50 \mu \mathrm{m}$. (d) Trajectories of cell centroids along the direction of the gradient for each cell denoted in (c). Negative $\mathrm{x}$ indicates movement towards stiffer region. (e,f) Histograms of centroid positions over 22 hour time course (initial positions set to $x=0$ ) of 10 cells that were initially in contact with stiffness boundaries on two identica discrete stiffness gradients and 4 cells on two hydrogel patterns with uniform stiffness. migrated toward the stiffer region (Fig. 3c). Trajectories of cell centroid migration along the direction of the gradient over the 22-hour time course were observed (Fig. 3d), and cells initially interacting with a stiffness boundary were biased toward the stiffer regions of the gradient (Fig. 3e), whereas cells plated on hydrogels with uniform stiffness spread in both directions (Fig. 3f). This result agrees with previously published data on durotaxis of NIH 3T3 mouse fibroblasts across a single-step stiffness gradient [3] or on a continuous stiffness gradient [10] where cells demonstrate directional migration towards the stiffer regions.

\section{CONCLUSION}

This study demonstrates a lithographic method for fabricating 3D ECM, enabling the control of cellular microenvironments with high precision and flexibility. Compared to previously used materials for controlling substrate stiffness, such as polydimethylsiloxane (PDMS) and polyacrylamide, use of a composite PEG-fibrinogen/PEGDA matrix allows patterning of substrates with a range of stiffness and spatial resolution that is observed in native tissues but has not yet been reproduced in vitro. Moreover, our approach can be applied to the creation of microstiffness patterns using other photopolymerizable hydrogels such as methacrylated hyaluronic acid and photocrosslinkable polyvinyl alcohol hydrogels. Overall, we believe the ability to micropattern celladhesive substrates exhibiting well-defined microscale variations in space and stiffness may allow the testing of hypotheses concerning the role of differential cell-ECM interactions in fundamental cell processes such as adherence, spreading, migration, and differentiation.

\section{REFERENCES}

[1] Yeung, T., et al. Effects of substrate stiffness on cell morphology, cytoskeletal structure, and adhesion. Cell Motil Cytoskeleton. 60(1):24-34, 2005.

[2] Pelham, R. J., Jr., and Y. Wang. Cell locomotion and focal adhesions are regulated by substrate flexibility. Proc Natl Acad Sci U S A. 94(25):13661-5, 1997.

[3] Lo, C. M., et al. Cell movement is guided by the rigidity of the substrate. Biophys J. 79(1):144-52, 2000.

[4] Wang, H. B., et al. Substrate flexibility regulates growth and apoptosis of normal but not transformed cells. Am J Physiol Cell Physiol. 279(5):C1345-50, 2000.

[5] Engler, A. J., et al. Matrix elasticity directs stem cell lineage specification. Cell. 126(4):677-89, 2006.

[6] Elkin, B. S., et al. Mechanical heterogeneity of the rat hippocampus measured by atomic force microscope indentation. J Neurotrauma. 24(5):812-22, 2007.

[7] Rho, J. Y., et al. Microstructural elasticity and regional heterogeneity in human femoral bone of various ages examined by nano-indentation. J Biomech. 35(2):189-98, 2002

[8] Zamir, E. A., et al. Mechanical asymmetry in the embryonic chick heart during looping. Ann Biomed Eng. 31(11):1327-36, 2003.

[9] Rains, J. K., et al. Mechanical properties of human tracheal cartilage. J Appl Physiol. 72(1):219-25, 1992.

[10] Zaari, N., et al. Photopolymerization in Microfluidic Gradient Generators: Microscale Control of Substrate Compliance to Manipulate Cell Response. Advanced Materials. 16(23-24):2133-21237, 2004.

[11] Almany, L., and D. Seliktar. Biosynthetic hydrogel scaffolds made from fibrinogen and polyethylene glycol for 3D cell cultures. Biomaterials. 26(15):2467-77, 2005.

[12] Dendukuri, D., et al. Continuous-flow lithography for high-throughput microparticle synthesis. Nature Materials. 5(5):365-369, 2006.

[13] Cheung, Y. K., et al. Direct patterning of composite biocompatible microstructures using microfluidics. Lab Chip. 7(5):574-9, 2007.

[14] Cheung, Y. K., et al. Microscale control of stiffness in a cell-adhesive substrate using microfluidics-based lithography. Angewandte Chemie. 121(39):7324-73287, 2009. 


\title{
3D Fibrous Matrices and their Cell Culture Performances
}

\author{
Y.T.1, H.W.1, C.W.1,2, A.S.1, T.L.1,* \\ 1 Centre for Material and Fibre Innovation, Deakin University, Geelong, VIC 3217, Australia \\ 2 Cytomatrix Pty. Ltd. Geelong, VIC3220, Australia \\ *Corresponding author’s email: tongl@deakin.edu.au
}

\begin{abstract}
In this paper, we have reported on a three dimensional (3D) fibrous matrix having excellent pore interconnectivity and controlled porosity, produced by a novel fiber assembly technique. Poly(E-caprolacton) (PCL) fibers (diameter 50 microns) were used to produce fiber matrices with porosity and pore size ranging from $73 \%$ to $97 \%$ and from $100 \mu \mathrm{m}$ to 1000 $\mu \mathrm{m}$, respectively. The $3 \mathrm{D}$ fibrous architectures were characterized by micro-computer tomography (micro-CT), scanned electronic microscope (SEM) and confocal microscope. The fibrous matrix had a stereoscopic framework with unique isotropic pore architecture. Hamster ovary cell (CHO) was used to examine effects of fibrous architectures on the cell culture performance. This new 3D scaffold might have an ability to integrate itself into a native tissue with the desired vascularization and controlled immune responses.
\end{abstract}

\section{INTRODUCTION}

Tissue engineering is a multidisciplinary field combining material engineering and life sciences towards the development of biological substitutes to restore, maintain or improve tissue functions [1, 2]. The key to engineering a tissue is a scaffold, a porous material that is used to support cell growth and proliferation into a well-integrated cell/scaffold composite initially, and the scaffold is then degraded and replaced by native extracellular matrix (ECM) so that a native tissue with desired biological functions is regenerated eventually [3].

In cell biology, interconnected pores are critical for a scaffold to be integrated with the surrounding tissues [4, 5]. In-vivo applications required that the pores in scaffold not only encourage cell growth, but also facilitate vascularisation for exchange of nutrients/waste with outside environment. In general, the former requires the pore size larger than the cells [6], while the later requires larger pores with excellent connectivity. For example, for formation of bone, the pores should be larger than $300 \mu \mathrm{m}$ [7]. Different cell types prefer to different pore sizes [8]. In addition, the pore size, orientation and morphology affect the cell attachment, adhesion and migration within the scaffold [4], as well as the regeneration of new tissue [9].

Nonwoven matrices are promising implant materials because of low manufacture costs and special porous structure. They have been developed for a wide range of medical applications, such as wound dressing, artificial skin, bone tissue, soft tissues, and heart valves[10-13]. Since the matrices are formed via physical assembly of fibers, fiber slippage always occurs when a mechanical perturbation is applied. As a result, pores in nonwoven matrix are poor in stability. Although this was improved via solvent or thermal bonding, the treatments also reduced the matrix porosity in the mean while.

In this paper, we reported on a novel fibrous tissue scaffolding material that have a stabilized porous structure and controlled pore size range, prepared by a combined meltbonding and template technique. Chinese hampster ovarian (CHO) was used to assess the biological performance of the fibrous matrices. The reason for choosing $\mathrm{CHO}$ cells is because that the $\mathrm{CHO}$ cells only grow in monolayer to reach confluence.

\section{EXPERIMENTAL}

PCL filaments were extruded on a Wayne Bantam single screw extrusion line. The fibers were then cut into short length and melt-bonded into a fibrous matrix in the presence of a template material (sugar powder). The discs used for cell cultures are $1.5 \mathrm{~cm}$ in diameter and $2 \mathrm{~mm}$ in thickness. Control PCL films were in the same size, but $0.85 \mathrm{~mm}$ in thickness.

In this work, a traditional static cell-seeding method was used to seed cells on the scaffold samples. After seeding, the cells were subjected to a static culture for 21 days. In the culture period, the media was first replenished after seven days of culture, and then repeated in each three days. For comparison, cell growing in the culture well only was used as a control as well. MTS assay was used to assess cell viability in vitro.

\section{RESULTS AND DISCUSSION}

Fig. 1 shows a typical SEM image of the fibrous matrix produced. All the fibers in the matrices showed no preferential direction and they were bonded in the contact parts thus maintaining stable pore architecture.

Image from the $\mu-C T$ showed that the matrix had an excellent porous structure. All pores in the matrix were wellinterconnected without any dead and end pores. The pores in matrix were oriented randomly and no preferably oriented pores were observed, which facilitate the nutrient transport across the scaffold and the formation of neo-tissues. Size of pores ranged from $100 \mu \mathrm{m}$ to $1000 \mu \mathrm{m}$. 

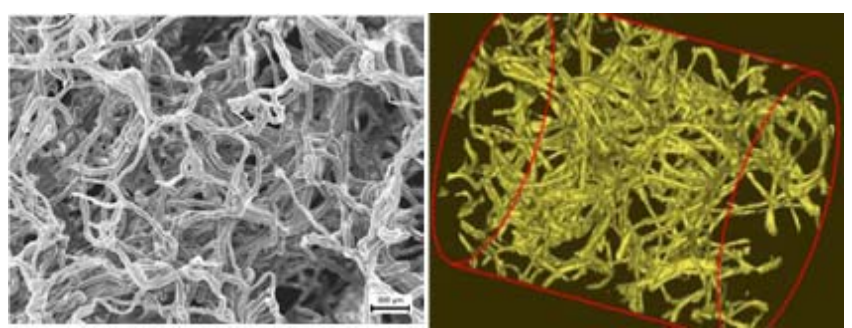

Fig. 1: SEM and $\mu-C T$ images of a 3D fibrous matrix.

Fig. 2 shows the confocal microscopic images of $\mathrm{CHO}$ cells growing inside a fibrous matrix. Variable cells distributed on sliced image at different depth of the matrix (not shown in this paper), indicating that the $\mathrm{CHO}$ cells went deep into fibrous matrix during culture. This could be further confirmed by micro-CT images (Fig. 2 down), Red spots inside the 3D structure are OsO4-stained cells.

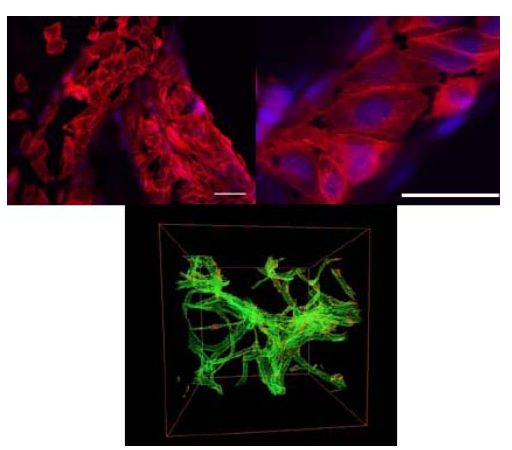

Fig. 2: (up) Confocal image of CHO cells on a fiber matrix (Scale

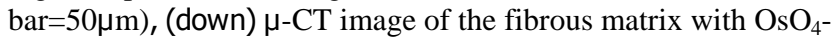
stained CHO cells.

Fig. 3 shows the mean number of $\mathrm{CHO}$ cells on the scaffold materials. 3D scaffolds were much better than 2D petri dish control and 2D film control samples after 7 days of culture. The speed of cell proliferation slowed down since 2 week for 2D control samples, but cells on 3D fibrous matrix kept growing in a stable speed till end of 21 days.

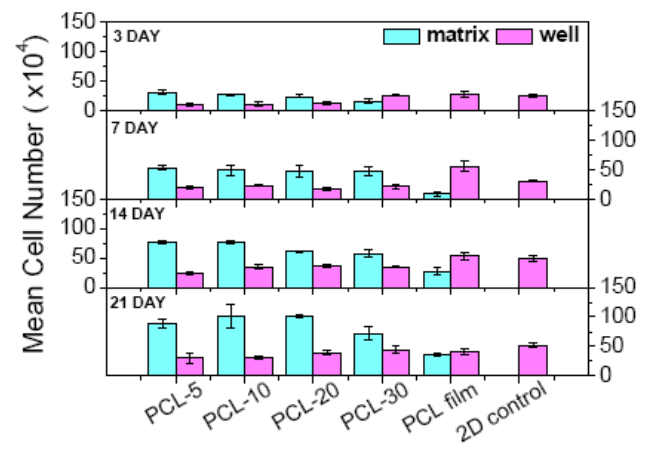

Fig. 3: Mean number of cells on different scaffolds.

\section{CONCLUSIONS}

We have demonstrated that a fibrous matrix produced by a novel fiber assembly technique by combining melt-bonding with a template method shows great potential to produce structure-stabilized fibrous matrix for 3D tissue scaffolding applications. The fibrous scaffolds can have different pore sizes and surface areas, and porosities.

The cell culture test indicated that $\mathrm{CHO}$ cells liked to grow and proliferate on the PCL fibrous matrices much more than the 2D film and 2D PS Petri dish; and the pore characteristic influenced the cell growth. All the fibrous matrices could be used potentially for further studied both in vitro and in-vivo for culturing cells and tissues or biomedical applications.

\section{ACKNOWLEDGEMENT}

Funding support from Australian Research Council (ARC Linkage, LP0776751) and Deakin University under the Centre Research Grant Scheme is acknowledged

\section{REFERENCES}

[1] Langer R, Vacanti JP. Tissue engineering. Science,vol.260, pp.920-926,1993.

Nerem RM, Sambanis A.,"Tisse engineering: From biology to biological substitutes,"Tissue Engineering,vol.1,pp.3-13,1995

[3] Mooney DJ, Vacanti JP,"Tissue engineering using cells and synthetic polymers,"Transplantation Reviews, vol.7, pp. 153-162,1993.

[4] Van Tienen TG, Heijkants RGJC, Buma P, De Groot JH, Pennings AJ, Veth $\mathrm{RPH}$,"Tissue ingrowth and degradation of two biodegradable porous polymers with different porosities and pore sizes,"Biomaterials,vol.23,pp.1731-1738,2002. 2002;23.

[5] Otsuki B, Takemoto M, Fujibayashi S, Neo M, Kokubo T, Nakamura T. Pore throat size and connectivity determine bone and tissue ingrowth into porous implants: Three-dimensional micro-CT based structural analyses of porous bioactive titanium implants. Biomaterials 2006;27(35):5892-5900.

[6] Akay G, Birch MA, Bokhari MA,"Microcellular polyHIPE polymer supports osteoblast growth and bone formation in vitro,"Biomaterials ,vol. 25, pp. 39914000, 2004.

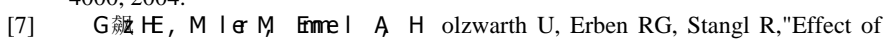
surface finish on the osseointegration of laser-treated titanium alloy implants,"Biomaterials,vol.25,pp.4057-4064, 2004.

[8] Zeltinger J, Sherwood JK, Graham DA, M Ile R, G iffith LG, " Effect of Pore Size and Void Fraction on Cellular Adhesion, Proliferation, and Matrix Deposition," Tissue Engineering,vol.7,pp.557-572,2001.

[9] I. V. Yannas,"Applications of ECM analogs in surgery," Journal of Cellular Biochemistry,vol.56,pp. 188-191,1994.

[10] Wang CC, Su CH, Chen CC,"Water absorbing and antibacterial properties of Nisopropyl acrylamide grafted and collagen/chitosan immobilized polypropylene nonwoven fabric and its application on wound healing enhancement," Journal of Biomedical Materials Research - Part A,vol.84,pp. 1006-1017,2008.

[11] Dal Pra I, Chiarini A, Boschi A, Freddi G, Armato U," Novel dermo-epidermal equivalents on silk fibroin-based formic acid-crosslinked three-dimensional nonwoven devices with prospective applications in human tissue engineering/regeneration/repair," International journal of molecular medicine,vol.18, pp.241-247, 2006.

[12] Ma PX, Langer R,"Morphology and mechanical function of long-term in vitro engineered cartilage," Journal of Biomedical Materials Research,vol.44,pp.217221,1999.

[13] Engelmayr Jr GC, Sacks MS,"Prediction of extracellular matrix stiffness in engineered heart valve tissues based on nonwoven scaffolds," Biomechanics and Modeling in Mechanobiology,vol.7,pp.309-321, 2008. 


\title{
The Influence of Architecture on the Bioactive and Osteogenic Properties of PCL-Ceramic Composites
}

\author{
Ajitha Patlolla, Treena Livingston Arinzeh \\ Department of Biomedical Engineering \\ New Jersey Institute of Technology, Newark, NJ
}

\begin{abstract}
The two most commonly investigated materials for bone repair are $\beta$-tricalcium phosphate $\left(\beta\right.$-TCP) $\left(\mathrm{Ca}_{3}(\mathrm{PO4})_{2}\right)$ and synthetic hydroxyapatite $(\mathrm{HA})\left(\mathrm{Ca}_{10}(\mathrm{PO4})_{6}(\mathrm{OH})_{2}\right)$ since they are bioactive apart from being biocompatible and osteoconductive [1]. These materials are categorized as bioactive materials as the implants of these materials have the ability to form strong chemical bond with the neighboring living bone when in contact with physiological fluid. Clinically, however, these materials have had limited use because of their brittleness and difficulty in shaping [2]. Therefore, biodegradable polymer/bioceramic composites have been sought as an alternative form to using calcium phosphates alone. In this study, composites consisting of varying weight percentages of $20 / 80 \mathrm{HA} / \mathrm{TCP}$ and poly ( $\varepsilon$ caprolactone) (PCL) were fabricated using the electrospinning technique. Two types of electrospun ceramic composites were fabricated and evaluated for bioactive and osteogenic properties. The results suggested that one of the composites had better bioactivity and osteogenic properties.
\end{abstract}

\section{INTRODUCTION}

The success of the orthopedic implant depends on its ability to interact with the surrounding bone. Biomaterials like hydroxyapatite, bioglass, and some other calcium phosphates are categorized as bioactive materials as the implants of these materials have the ability to form strong chemical bond with the neighboring living bone when in contact with physiological fluid. The surface region of these materials is transformed to carbonate apatite through a series of surface reactions including dissolution, precipitation and ion exchange when inserted into the body [3]. Formation of carbonate apatite on the implant surface in the body is believed to be one of the events leading to form a strong chemical bond between implant and bone [4, 5]. Kokubo proposed that the in-vivo bioactivity of these materials can be predicted by testing these materials in-vitro by immersing them in simulated body fluid (SBF) and examining the formation of apatite that is similar to bone apatite on the surface of biomaterials over a period of time [6].

MSCs are able to differentiate into various cells including chondrocytes, osteoblasts, and adipocytes. However, it is important to determine how the scaffolds direct MSCs towards a particular cell type. In order to asses the scaffolds, the expression of bone specific genes will be tested on them by growing MSCs on the scaffolds and conducting gene expression studies. Bone formation involves differentiation of MSCs to preosteoblasts followed by their maturation to osteoblasts, which produces unmineralized ECM followed by its calcification. During these various stages, the cells produce different matrix proteins, type I collagen and non-collagenous proteins.
The two most commonly investigated materials for bone repair are $\beta$-tricalcium phosphate $\left(\beta\right.$-TCP) $\left(\mathrm{Ca}_{3}(\mathrm{PO} 4)_{2}\right)$ and synthetic hydroxyapatite $(\mathrm{HA})\left(\mathrm{Ca}_{10}(\mathrm{PO} 4)_{6}(\mathrm{OH})_{2}\right)$ since they are biocompatible, osteoconductive, and bioactive [1]. Clinically, however, ceramics have had limited use because of their brittleness and difficulty in shaping [2]. Therefore, biodegradable polymer/bioceramic composites have been sought as an alternative form to using calcium phosphates alone. In this study, composites consisting of varying weight percentages of $20 / 80 \mathrm{HA} / \mathrm{TCP}$ and poly ( $\varepsilon$-caprolactone) (PCL) were fabricated using the electrospinning technique. Solvent and solvent combinations were evaluated to form scaffolds with a maximum concentration of ceramic and uniform dispersion. The fabricated electrospun composites were evaluated for bioactive and osteogenic properties.

\section{EXPERIMENTAL METHODS}

A. Materials: poly (€-caprolactone) (Sigma Aldrich, 80,000 MW), nano hydroxyapatite and nano beta tricalcium phosphate with average size $100 \mathrm{~nm}$ (Advanced Biomaterials) were used for fabricating scaffolds. The solvents used for processing were methylene chloride and dimethylformamide

B. Scaffold Fabrication: The scaffolds were fabricated using the electrospinning process. A $10 \mathrm{ml}$ plastic disposable syringe, 20 gauge needle, voltage of $15 \mathrm{KV}$, flow rate of $3 \mathrm{ml} / \mathrm{hr}$ and a distance of $15 \mathrm{~cm}$ were the parameters used for fabricating the electrospun scaffolds. The composite solution was prepared by ultrasonicating the suspension of solvent and ceramic for one minute, followed by the addition of polymer $(10 \% \mathrm{PCL} \mathrm{w} / \mathrm{w})$ to the suspension, which was stirred for 24 hours at $300 \mathrm{rpm}$ at room temperature.

C. Simulated Body Fluid Preparation: A simulated body fluid (SBF) with ion concentrations nearly equal to those of human blood plasma was prepared following Kokubo's method [6]. The SBF was prepared by dissolving the reagents into the pure water at $37^{\circ} \mathrm{C}$ one by one. Finally, it was adjusted to a $\mathrm{pH}$ of 7.4 at $37^{\circ} \mathrm{C}$ with HCL. The samples were then soaked in SBF solution at $37^{\circ} \mathrm{C}$ for various time points, day 7 , day 14 , day 21 , and day 28 . Each sample was cut into $50 \mathrm{~mm} \mathrm{X}$ $50 \mathrm{~mm}$ and soaked in $20 \mathrm{ml}$ of SBF and placed in incubators. The media was replaced every third day. After the predetermined soaking time, the samples were removed from the SBF solution, gently rinsed with distilled water and dried at room temperature.

C. Characterization of immersed samples: The apatite formation and its morphology were visually observed using scanning electron microscopy (SEM, LEO 1530 Gemini, Germany). SEM-energy dispersive X-ray analysis (SEMEDXA) was performed on the apatite formed on the samples 
to determine the elemental composition of the apatite. X-ray diffraction of the SBF soaked samples were collected using X'pert Pro Diffractometer (PW3050/60, Philips, Netherlands). The apatite formation on immersed samples was analyzed using fourier transformed infrared spectrometer (Perkin Elmer FTIR). The infrared spectrum was collected in the range of $4000-400 \mathrm{~cm}^{-1}$ with a resolution of 4 and scans of 32 .

D. Gene Expression Studies: The scaffolds were evaluated for the expression of Runx2, Type I collagen and Osteopontin genes by conducting RT-PCR studies on day 14 after cell seeding in growth media and induction media.

\section{RESULTS AND DISCUSSIONS}

A. Materials Fabrication: The fibers formed were uniform up to $30 \%$ ceramic concentration but non-uniformity and agglomerates of the ceramic formed starting at $40 \%$ ceramic concentrations. Therefore, 30\% ceramic composites were further used for testing their bioactive properties and conducting in-vitro cell studies.

B. Characterization of immersed samples: The SEM images of the SBF immersed samples; electrospun PCL, 30\% Composite-MC and 30\% Composite-MC+DMF are shown in the figure 1. The SEM results suggest that there is no visually appearing apatite formation on only PCL electrospun sample (fig 1(a, b)), whereas apatite formation was seen on the ceramic composites. However, the apatite formation is significantly more on the Composite-MC (fig $1(\mathrm{e}, \mathrm{f}))$ than on the Composite-MC+DMF (fig 1(c, d)). The EDXA, XRD and FTIR results also confirmed that there is no apatite formation on the PCL surface but there is apatite formation on the composites.

The XRD data of Composite-MC and Composite$\mathrm{MC}+\mathrm{DMF}$ samples immersed in SBF is shown in the figure 2. The intensity of peaks corresponding to hydroxyapatite (34.1, $32.9,32.2,31.8,28.9,28.1)$ increased over time and the intensity of the peaks corresponding to beta-TCP $(34.3,30.9$, 27.7 , 26.4) reduced over time in the composite samples, suggesting apatite formation on the composite samples. The intensities of the peaks also suggest that the amount of apatite formed on Composite-MC is more than Composite-MC+DMF samples. The XRD data also found that there is no apatite formation on the PCL samples as there were no extra peaks formed after the immersion.

The FTIR data also confirmed the formation of the apatite on the surface of Composite-MC and Composite$\mathrm{MC}+\mathrm{DMF}$ whereas there is no apatite formation on unfilled PCL electrospun mats (figure 3). The intensities of the peaks corresponding to hydroxyapatite (1021 and $\left.559 \mathrm{~cm}^{-1}\right)$ increased and the intensities of the peaks corresponding to PCL (1720 and $1156 \mathrm{~cm}^{-1}$ ) decreased as the time of soaking in SBF increased. The intensities of the apatite peaks in Composite-MC are higher than in the Composite-MC+DMF peaks, which suggest that the Composite-MC has more bioactive properties compared to Composite-MC+DMF.

C. Gene Expression Studies: The Runx2 gene, Type I collagen and Osteopontin genes were expressed on Day 14, where as Sox2 gene was not expressed, suggesting there were no significant number of MSCs left on day 14 (Figure 2). The expression of Runx2 was high on the Composite-MC mats.

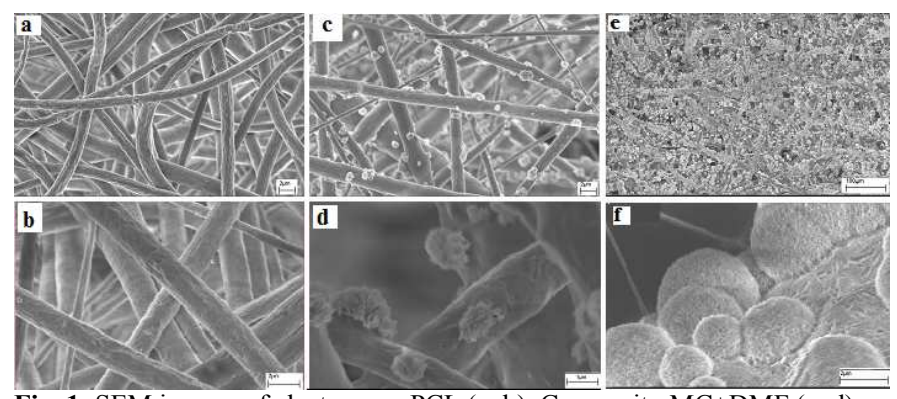

Fig. 1: SEM images of electrospun PCL (a, b), Composite-MC+DMF (c, d) and Composite-MC (e, f) immersed in SBF for 28 days at lower and higher magnification

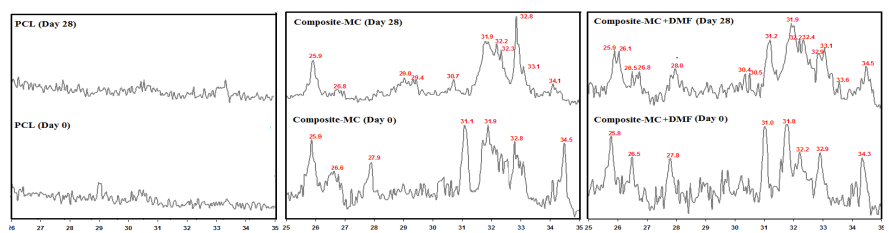

Fig. 2: XRD data of a) electrospun PCL b) Composite-MC c) Composite$\mathrm{MC}+\mathrm{DMF}$ samples immersed in SBF for 28 days

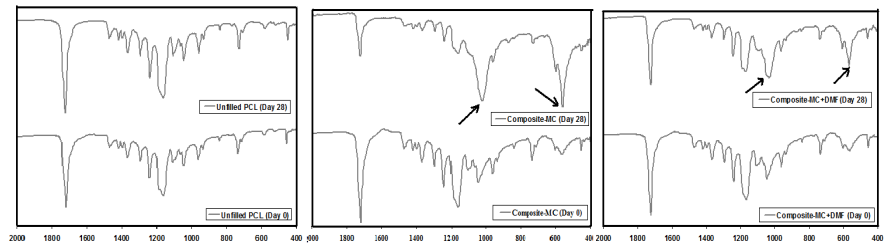

Fig. 3: FTIR Spectrum of a) electrospun PCL b) Composite-MC and c) Composite-MC+DMF samples immersed in Simulated Body Fluid (SBF) for 28 days

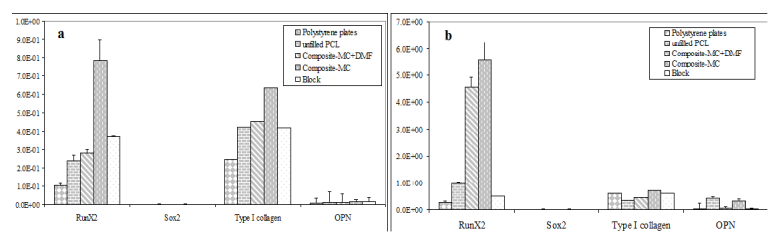

Fig. 4: Gene expression data of MSCs in growth media (a) and induction media (b)

\section{CONCLUSION}

Electrospun composites fabricated using different solvents resulted in varying fiber diameters and pore sizes which resulted in varying bioactivity properties and gene expression. Composite-MC demonstrated more favorable bioactive properties and gene expression. This suggests that the novel composite scaffold with larger pore sizes, high crystallinity and novel architecture will prove to be a promising scaffold for bone tissue engineering.

\section{REFERENCES}

[1] Z. Zhang et al., Oral science International. 2005; 85-95

[2] M. Wang et al., Biomaterials 2003; 24:2133-51

[3] S.B. Cho. J. Et al., Mater. Sci.: Mater. Med. 9 (1998) 279

[4] P. Ducheyne, et al., Cuckler, Clin. Ortop. 276 (1992)102

[5] L.L. Hench et.al., J. Am. Ceram. Soc. 74 (1991) 1487

[6] L. Kokubo, Biomaterials 12 (1991) 155 


\title{
Use of GAG-like polysaccharides to engineer hydrogel-filled nanofibrous structures
}

\author{
Piyush Modak, Asya Bakhtina, Gloria Portocarrero, Treena Arinzeh, Willis Hammond, George Collins \\ Department of Biomedical Engineering \\ New Jersey Institute of Technology \\ Newark, NJ 07102
}

\begin{abstract}
The goal of this study is the complete simulation of the structural and the functional components of the extra-cellular matrix using semi-synthetic polysaccharides for tissue engineering applications
\end{abstract}

\section{INTRODUCTION}

The extracellular matrix (ECM) consists of fibrous proteins like collagen, elastin, etc., which provide structural support and sites for attachment for the cells. This fibrous network is fully immersed in a hydrogel composed of proteoglycans - a protein core with glycosaminoglycan (GAG) side chains. GAG's are functionally important for the presentation of proteins (cytokines, growth factors) to cell receptors [1]. Our aim is the complete simulation the structural \& functional components of the ECM. Electrospinning is widely used to prepare nonwoven fibrous mats with diameters in the submicron range from natural and synthetic polymers [2]. Gelatin - denatured form of collagen can be electrospun and crosslinked to simulate the fibrous, structural component [3]. Naturally occurring polysaccharides like cellulose and dextran when sulfated, mimic the chemical structure of GAGs [7]. Hydrogels formed from cost effective, semi-synthetic sulfated polysaccharides will be used to fill the interstices of fibrous mats and mimic the function of a GAG component.

In the present study our principal effort is to crosslink these semi-synthetic sulfated polysaccharides using physical as well as chemical crosslinking techniques in order to render them physically stable under the conditions of cell incubation.

\section{EXPERIMENTAL MATERIALS AND METHODS}

A. Materials used:

Sodium cellulose sulfate (NaCS), Dextran, Sodium dextran sulfate $(\mathrm{NaDexS})$, and Diethylaminoethyl (DEAE) Dextran were generously gifted by Dextran Products ltd, Onatrio, Canada. Chitosan, polydiallyldimethylammonium chloride (PDADMAC) and Calcium Chloride Dihydrate $\left(\mathrm{CaCl}_{2}\right.$. $2 \mathrm{H}_{2} \mathrm{O}$ ), were purchased from Sigma Aldrich, Texas. Dulbecco's Modified Eagle Medium (DMEM), Phosphate
Buffered Saline (PBS) were purchased from Invitrogen Co. (Carlsbad, CA). The sulfur content of $\mathrm{NaCS}$ is $18.2 \%$ and the $\mathrm{NaDexS}$ is $7.8 \%$.

\section{B. Physical Crosslinking:}

\section{1) Ionic Crosslinking(divalent ions):}

Ionic crosslinking involves the exchange of divalent ions with the monovalent sodium cation to form a stable gel. Alginate has been shown to crosslink using $\mathrm{BaCl}_{2}$ and $\mathrm{CaCl}_{2}$ solution [4]. NaCS was similarly crosslinked using $\mathrm{Ba}^{2+}$ and $\mathrm{Ca}^{2+}$ ions. $5 \%$ aq. solution of $\mathrm{NaCS}$ was dripped in a $30 \%$ $\mathrm{BaCl}_{2}$ aq. solution and in $74 \% \mathrm{CaCl}_{2}$ aq. solution. Both $\mathrm{Ba}^{2+} \&$ $\mathrm{Ca}^{2+}$ crosslinked NaCS gels were immersed in DI water to check for stability.

2) Polyelectrolyte complexes(multivalent ions):

Polyelectrolyte complexes are formed by forming a coacervate between a polyanion and a polycation [5]. Thin polyelectrolyte membranes can be formed by the layer-bylayer technique by depositing a layer of a polyanion, on top of polycation [6]. Three different membranes were prepared by depositing a $5 \mathrm{wt} \% \mathrm{NaCS}$ solution on PDADMAC, DEAE Dextran and Chitosan. PDADMAC, DEAE Dextran were at a $20 \%$ concentration in water while a $7 \%$ chitosan solution was prepared in $90 \%$ acetic acid and water. The membranes were prepared in 24-well ultra low well attachment polystyrene plates and checked for stability in DMEM at $37^{\circ} \mathrm{C}$ in the incubator for 4 weeks. Media was changed every 3 days and membranes were washed with PBS before adding fresh media.

Human Mesenchymal Stem Cells (hMSC's) were seeded on NaCS-PDADMAC membranes and images were taken using confocal microscopy 4 days after incubation in DMEM. Actin/DAPI stain was used to stain the nucleus and the cytoskeleton.

\section{Chemical Crosslinking:}

Hydrogels that are covalently-crosslinked networks are chemical gels [5]. Dextran and partially sulfated Dextran Sulfate were crosslinked using an isosorbide based epoxy crosslinker. Solutions of $20 \%$ of Dextran and $24.6 \%$ partially sulfated $\mathrm{NaDexS}$ in $1 \mathrm{M} \mathrm{NaOH}$ were made. $20 \%$ of the 
crosslinker wrt the wt \% of each polymer was added and the temperature was raised to $50^{\circ} \mathrm{C}$.

\section{RESULTS}

\section{A. Physical Crosslinking:}

1) Ionic Crosslinking:

$\mathrm{NaCS}$ crosslinked using $\mathrm{CaCl}_{2}$ solution dissolved in water after 5 mins. But $\mathrm{Ba}^{2+}$ crosslinked $\mathrm{NaCS}$ gel was stable in DI water for over a week.

\section{2) Polyelectrolyte Complexes:}

All three membranes remained intact after 4weeks incubation in the media.

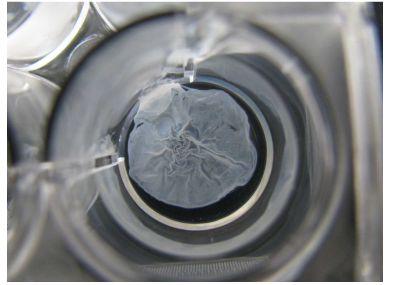

(a)

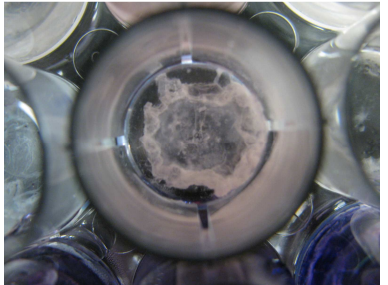

(b)
Fig. 1: Images of NaCS-PDADMAC films (a) before incubation and (b) after incubation.

Fig. 2 is the confocal image at $60 \mathrm{X}$ magnification of the hMSc's seeded onto the NaCS-PDADMAC membrane four days after incubation in the media.

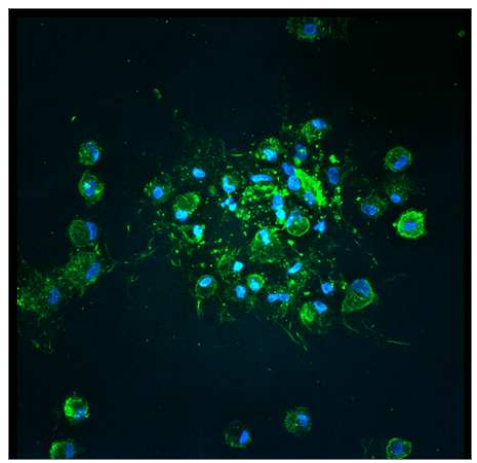

Fig 2: hMSC's on NaCS-PDADMAC membrane

The blue color (DAPI) indicates a compact nucleus and the green color is for the cytoskeleton.

\section{B. Chemical Crosslinking:}

The Dextran and the partially sulfated NaDexS solutions formed a gel within 5 minutes after the crosslinker was added and the temperature was raised to $50^{\circ} \mathrm{C}$. A piece of the gel was immersed in water. It swells in water and stays in its swollen state.

Fig.3 is the image of dextran hydrogel in its swollen state after 8 months in water.

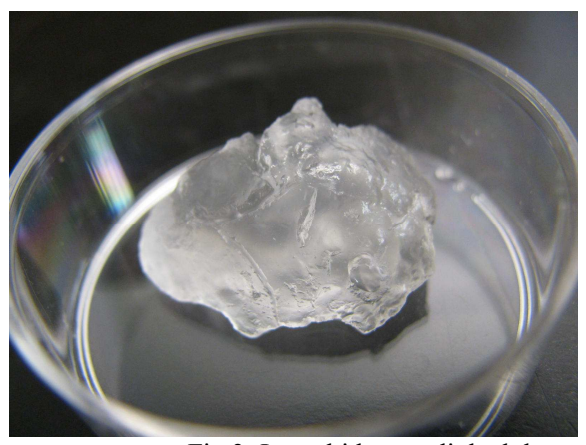

Fig 3: Isosorbide crosslinked dextran hydrogel

\section{SUMMARY}

The NaCS was crosslinked using physical crosslinking with the divalent cation $\mathrm{Ba}^{2+}$ ions and the polycationic PDADMAC, DEAE Dextran and Chitosan. Cell viability was determined on the NaCS-PDADMAC polyelectrolyte complex. Dextran and $\mathrm{NaDexS}$ were crosslinked using an isosorbide based epoxy crosslinking agent. The Dextran and NaDexS gels swell in water and remain stable.

\section{FUTURE GOALS}

Current ongoing experiments include crosslinking $\mathrm{NaCS}$ using a chemical crosslinker. And also to develop a nanofiber gelatin construct filled with a crosslinked sulfated polysachharide. The future goal of this project is to determine the ability of sulfated polysaccharides to function in a manner similar to GAGs in the presentation of growth factors for the development of cells.

\section{ACKNOWLEDGEMENT}

We would like to thank Dr. George Usher of Dextran Products for his unconditional support in our efforts.

\section{REFERENCES}

[1] Modrowsk D, Lomri A, Marie PJ. “Glycosaminoglycans bind granulocyte-macrophage colony-stimulating factor and modulate its mitogenic activity and signaling in human osteoblastic cells". Journal of Cellular Physiology 1998; 177(1):187-195

[2] Subbiah T, Bhat GS, Tock RW, Parameswaran S, Ramakumar SS. "Electrospinning of Nanofibers". Journal of Applied Polymer Science 2005;96(2):557-569

[3] Ju-Ha Song, Hyoun-Ee Kim and Hae-Won Kim "Production of electrospun gelatin nanofiber by water-based co-solvent approach". $J$ Mater Sci: Mater Med 2008; 19:95-102

[4] Taqieddin E., Amiji M., "Enzyme immobilization in novel alginatechitosan core-shell microcapsules." Biomaterials 2004; 25(10):19371945.

[5] Hoffman AS. "Hydrogels for biomedical applications". Advanced Drug Delivery Reviews 2002; 54(1):3-12.

[6] Schwarz H.-H., Richau K., Paul D., "Membranes from polyelectrolyte complexes" Polymer Bulletin 1992; 25: 95-100

[7] Owens GK, Geisterfer AA, Yang YW, Komoriya A. "Transforming growth factor-beta-induced growth inhibition and cellular hypertrophy in cultured vascular smooth muscle cells". J Cell Biol. 1988; 107(2):771780 


\title{
Electrospun Chitosan-Based Nanofiber Scaffolds for Cardiac Tissue Engineering Applications
}

\author{
Ali Hussain, George Collins, Cheul H. Cho \\ New Jersey Institute of Technology \\ 323 Martin Luther King Jr Blvd \\ Newark, NJ 07102
}

\begin{abstract}
The objective of this study was to fabricate 3-dimensional (3D) chitosan nanofiber scaffolds using an electrospinning technique and explore its potential for cardiac tissue engineering. Three culture conditions were tested: cardiomyocytes only, cardiomyocytes-fibroblasts cocultures, and cardiomyocyte-endothelial cells cocultures. The cells were seeded on 2-dimensional (2D) chitosan films and 3D chitosan nanofibers. Cellular morphology and functionality were assessed using immunofluorescent staining for alpha-sarcomeric actin (SA) and gap junction protein, connexin-43 (Cx43). In both the 2D and 3D scaffolds, only the cardiomyocyte-fibroblasts cocultures resulted in polarized cardiomyocyte morphology, fibril SA expression and Cx43 expression was higher compared to the other two conditions. In addition, the fibroblasts cocultures demonstrated synchronized contractions involving large tissue-like cellular networks. To our knowledge, this is the first attempt to utilize 3D chitosan nanofibers as cardiomyocyte scaffolds. Our results demonstrate that chitosan nanofibers can serve as a potential scaffold that can retain cardiomyocyte morphology and function.
\end{abstract}

\section{INTRODUCTION}

The in vitro generation of a 3-dimensional (3D) myocardial tissue-like construct employing cells, biomaterials, and biomolecules is one of the promising strategies in cardiac tissue engineering. Heart transplantation remains the final option for end-stage heart failure. However due to the scarcity of heart donors and the complications related to immune suppression and rejection, researchers are turning towards cardiac tissue engineering to discover effective solutions to revive the distressed heart.

The aim of this study is to demonstrate for the first time the potential of 3D chitosan nanofibers as scaffolds for cardiac tissue engineering applications. Chitosan is a natural polysaccharide biomaterial that is non-toxic, biocompatible, biodegradable, and cost effective. The chemical structure of chitosan is similar to the glycoasminoglycans in the extracellular matrix, and its hydrophilicity enhances its interaction with growth factors, cellular receptors, and adhesion proteins [1]. In order to enhance cellular adhesion, fibronectin was adsorbed onto the chitosan nanofibers before seeding.
Electrospinning is currently being extensively studied for tissue engineering applications because of its ability to produce nano-structures with a very high surface area to mass ratio (40 to $100 \mathrm{~m}^{2} / \mathrm{g}$ ) [2]. In addition, the fibrous structure forms a network of interconnected void space that provides a fibrous environment that is similar to the in vivo extracellular matrix.

In a healthy heart, more than half the cells are noncardiomyocytes, which include cardiac fibroblasts and endothelial cells. Fibroblasts have been shown to propagate electrical signal over distances of 100um via gap junctions and help synchronize cardiomyocyte contractions [3]. Endothelial cells enhance rapid vascularization of tissue engineered grafts which is especially vital for elevated oxygen consuming tissues such as the myocardium. Hence, cardiomyocytes were co-cultured with either fibroblasts or endothelial cells on the chitosan nanofibers and the expression of alpha sarcomeric actin (SA) and connexin-43 (Cx43) gap junction proteins were examined.

\section{METHODS}

An $8 \%$ (w/v) solution was prepared by dissolving chitosan (200kDa Sigma) in 80:20 trifluoroacetic acid: methylene chloride. The solution was stirred overnight at $40^{\circ} \mathrm{C}$. The distance between the 18 gauge needle and ground plate was $30 \mathrm{~cm}$, and $30 \mathrm{kV}$ was supplied by a DC voltage supply. The solution feeding rate was set to $3 \mathrm{ml} / \mathrm{hr}$. After spinning, the electrospun scaffold was dried at $50^{\circ} \mathrm{C}$ overnight to eliminate any remaining solvent. The scaffold was then neutralized in 1:1 ( $15 \mathrm{~N}$ ammonium hydroxide and ethanol) for 30 minutes, followed by 30 minutes distilled water incubation. The scaffold was then sterilized with UV for 15 minutes and incubated in $10 \mathrm{ug} / \mathrm{ml}$ fibronectin solution for 1 hour. A fibronectin adsorbed chitosan film was used as a $2 \mathrm{D}$ control.

$5 \times 10^{5}$ ventricular neonatal rat cardiomyocytes were suspended in $0.3 \mathrm{ml}$ of cardiomyocytes media and then seeded onto the scaffold. The scaffold was incubated for 2 hours at $37^{\circ}$ $\mathrm{C}$ to allow the cells to attach. An additional $1.2 \mathrm{ml}$ media was then added to the tissue culture plate. In the coculture systems, the different cell types were mixed together in a 1:1 ratio prior to seeding $\left(5 \times 10^{5}\right.$ cells of each type). Similar cell seeding 
procedure was followed for the fibronectin adsorbed 2D chitosan films.

\section{RESULTS}

Scanning electron microscopy (SEM) images of the electrospun chitosan nanofibrous scaffold indicated that the average fiber diameter to be 250nm (Fig1).

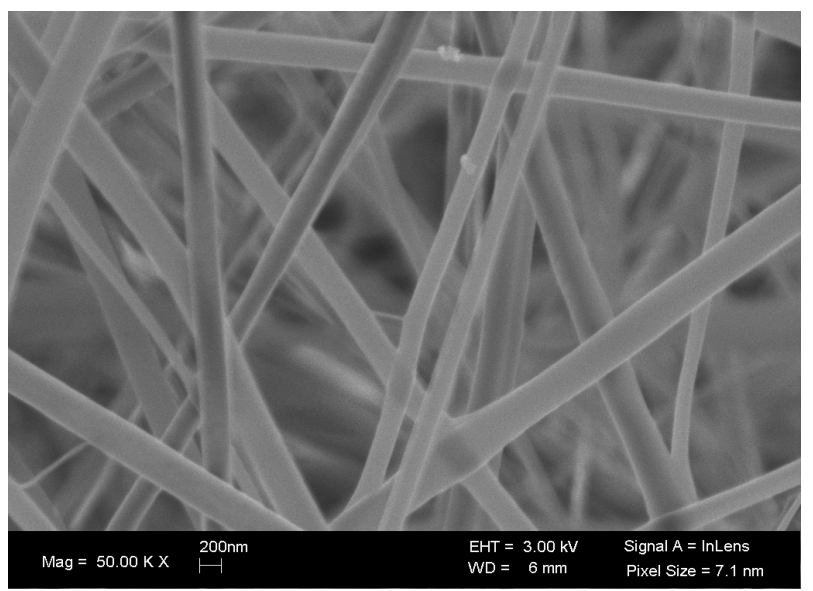

Fig. 1. SEM image of electrospun chitosan fibers at 50,000 magnification.

On the 2D chitosan film scaffolds cardiomyocyte monocultures resulted in loss of cardiomyocyte morphological polarity, minimal Cx43 expression and islands of non-coherent contractions. The cardiomyocyte-fibroblasts cocultures resulted in interconnected tubular networks which exhibited synchronized contractions and the highest SA and Cx43 expression. Cardiomyocyte-endothelial cells cocultures resulted in contracting circular myocyte clusters, with minimal SA and Cx43 expression. In 3D chitosan nanofiber scaffolds, the cardiomyocyte monoculutres resulted in spherical cell morpholpogy. The cardiomyocyte-fibroblasts coculutres resulted in elongated networks of contracting cardiomyocytes and elevated SA and Cx43. The cardiomyocyte-endothelial cells cocultures resulted in spherical cardiomyocytes with low levels of $\mathrm{Cx} 43$ expression.
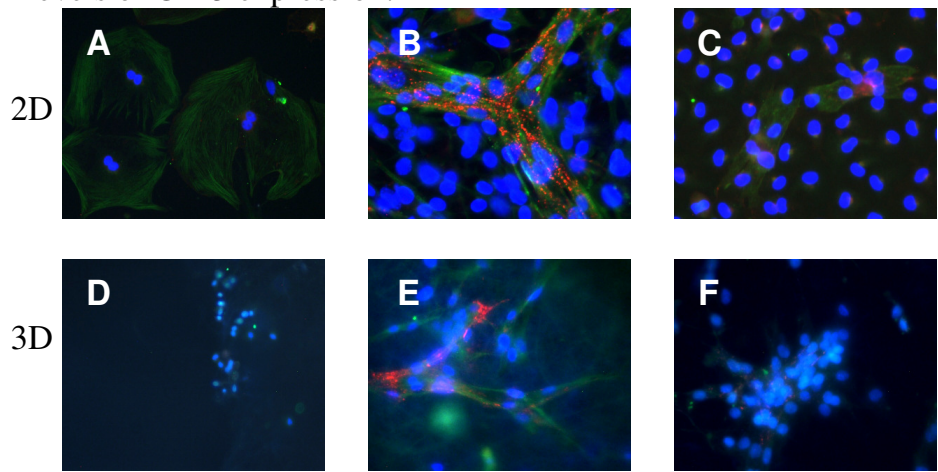

Fig. 2. Immunofluorescence staining of cardiomyocytes culture alone (A,D), cardiomyocyte-fibroblasts coculture $(\mathrm{B}, \mathrm{E})$, and cardiomyocyte-endothelial cells coculture (C,F). Top row: 2D cultures on chitosan films (day 7); Bottom row: 3D in chitosan nanofibers (day 19). Cells were labeled with SA (green), Cx43 (red), and DAPI (blue).

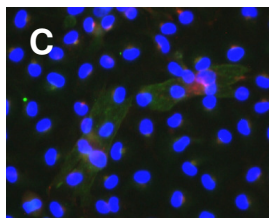

\section{DISCUSSION}

This is the first study to test chitosan nanofibers as potential scaffolds in the field of cardiac tissue engineering. The results demonstrate that primary cardiomyocyte viability and functionality can be sustained on chitosan nanofibers in vitro for longer time periods when cocultured with fibroblasts (up to 4 weeks).

Cardiac fibroblasts are the most abundant non-cardiomyocyte cells in the mature heart. Their functions include deposition of the extracellular matrix (ECM), paracrine signaling and propagation of the electrical stimuli. SA expression is solely found in cardiomyocytes and was present in all cultures containing cardiomyocytes. $\mathrm{Cx} 43$ is the gap junction protein that is mainly found in ventricular cardiomyocytes [1]. The Cx43 mediates fibroblasts heterogeneous coupling, such as between cardiomyocytes and fibroblasts [4]. These gap junctions with fibroblasts are known to propagate electrical stimuli for $100 \mu \mathrm{m}$ [1]. The results from both the 2D and 3D cultures indicate that fibroblasts cocultures resulted in the highest SA and Cx43 expression suggesting fibroblasts are essential in maintaining cardiomyocytes viability and function in vitro.

The observation that cardiomyocytes are able to survive, express key functional proteins, and undergo synchronized contractions on chitosan nanofibers has never been reported before, as far as we were able to determine. These chitosan nanofiber scaffolds can be layered on top of each other to create a thick tissue-like structure composed of cardiomyocytes, fibroblasts and endothelial cells. The fibroblasts will enhance the electrical synchronization of the cardiomyocytes, while the endothelial cells will encourage vascularization into the graft. Further characterization of the chitosan nanofiber scaffolds is needed in terms of electrophysiological measurements and mechanical properties.

\section{ACKNOWLEDGEMENT}

We thank Dr. Junichi Sadoshima (UMDNJ) for the gift of rat neonatal cardiomyocytes. This study was supported by NJIT startup fund.

\section{REFERENCES}

[1]
Blan, N. and R. Birla, "Design and fabrication of heart muscle using scaffold-based tissue engineering," Journal of Biomedical Materials Research Part A, vol. 86, pp 195-208. July 2008

Desai, K., et al., "Morphological and surface properties of electrospun chitosan nanofibers," Biomacromolecules, vol. 9, pp1000-1006. January 2008.

Gaudesius, G., et al., "Coupling of cardiac electrical activity over extended distances by fibroblasts of cardiac origin," Circulation Research, vol 93, pp421-428. July 2003.

Snider, P., et al., "Origin of Cardiac Fibroblasts and the Role of Periostin.," Circulation Research, vol 105, pp 934-947. November 2009. 


\title{
Effect of wear-debris particles on RAW 264.7 cells
}

\author{
M. K. Musib, S. Saha \\ Department of Orthopedic Surgery and Rehabilitation Medicine \\ SUNY Downstate Medical Center \\ Brooklyn, New York 11203 \\ mrinal.musib@downstate.edu
}

\begin{abstract}
Wear-debris particles from ultrahigh molecular weight polyethylene (UHMWPE) and metals has been implicated in periprosthetic osteolysis and cellular response to them depend on their size and dose. Response to larger particles has been previously studied, but there are no specific studies examining biological response to simultaneous administration of both UHMWPE and Ti particles less than $0.2 \mu \mathrm{m}$ in size. Our lab has developed a standardized and reproducible technique to isolate, characterize and fractionate both particles into 3 distinct size ranges [1.0-10.0 $\mu \mathrm{m}$ (micron), $0.2-1.0 \mu \mathrm{m}$ (submicron) and $0.01-0.2 \mu \mathrm{m}$ (nano)] and furthermore minimize clumping, which facilitated disaggregation and isolation of individual particles. RAW 264.7 cells were treated for $24 \mathrm{~h}, 48 \mathrm{~h}$ and $72 \mathrm{~h}$ with various doses and sizes of both particulates simultaneously. Cell membrane damage was measured by quantifying lactate dehydrogenase (LDH) release. The cells elicited a dose and size dependent response to the nano-particulates at the highest dose. At $72 \mathrm{~h}$ there was a significant increase in $\mathrm{LDH}$ released by cells treated with the nanoparticles as compared to untreated controls as well as to larger particles. To mimic in vivo conditions, further studies are being conducted and will involve administration of dual wear particulates that will help understand cellular response to wear particulates.
\end{abstract}

\section{BACKGROUND}

Although wear particulates from polyethylene and metals are responsible for osteolysis, isolation and fractionation of 'clean' wear-debris particles from complex biological media, such as those used in wear simulator machines, has been a longstanding problem. Previous isolation techniques have been associated with the potential loss of nanoparticles during isolation. Understanding how cells respond to particles $<0.2 \mu \mathrm{m}$ is important as previous studies have implicated smaller particles in wear-mediated osteolysis and that cellular response to particles is dependent on size and dose [1-8].

\section{METHODS}

In this study, we combine the use of an optimized solvent with mechanical treatment for reducing particle aggregation and improve particle recovery. Fractionated particles (micron-, sub-micron-, and nano-size fractions) were then added to cell cultures to examine their effect on phagocytosis and membrane integrity of RAW 264.7 murine monocyte/macrophage cell line. The overall hypothesis of the current work is that RAW 264.7 cells (which have been previously used to study cellular response to wear particles) $[9,10]$ will display a more robust response to nanaoparticles as compared to larger particles and that simultaneous coadministration of both UHMWPE and Ti particles will be more deleterious to cells than those displayed to individual component.

A. Recovery and fractionation of UHMWPE particles into 3 different size ranges: UHMWPE (GUR 1050) and $\mathrm{Ti}$ particles were obtained from Ticona and Alpha Aesar (\#681) respectively. Particles were suspended in acidified water (pH 5.5) containing Pluronic (2000 ppm); suspensions were vortexed for 5 minutes, sonicated for 120 minutes and filtered through a $10 \mu \mathrm{m}$ pore size filter to remove large aggregates. Particulates were separated into three different sizes; micron (1-10 $\mu \mathrm{m})$, submicron $(0.2-1 \mu \mathrm{m})$ and nano(0.01-0.2 $\mu \mathrm{m})$ using filters of varying pore size. Representative SEM images were obtained; particle number and size-shape were determined. Particulate suspensions were prepared containing varying concentrations $\left(10^{3}, 10^{5}\right.$, $10^{7}$ per $\mathrm{mL}$ ) of either of the particles in $10 \%$ fetal bovine serum (FBS).

B. Phagocytosis: RAW 264.7 cells were cultured to confluence, and then treated with varying doses of the fractionated UHMWPE/Ti particles for $24 \mathrm{~h}$. At harvest, cell layers were washed with PBS, collected by centrifugation, fixed with glutaraldehyde and then postfixed using $\mathrm{OsO}_{4}$. Samples were embedded in Epon, sectioned, mounted on copper grids, stained with $1 \%$ uranyl acetate/lead citrate and examined in the TEM.

C. Cell membrane integrity: Cells were cultured to confluence and then treated with varying doses of particles for each of the 3 size ranges. After $72 \mathrm{~h}$, cultures were harvested and analyzed using Cytotox-ONE assay kit (Promega). The positive control had all cells lysed using dilute Triton solution.

D. Statistical Interpretation of Data: ANOVA was used to analyze the data; post-hoc testing was performed using Student's t-test with Bonferonni correction. $\mathrm{P}$ values $\leq 0.05$ were considered significant.

\section{RESULTS}

Recovery and fractionation of UHMWPE particles: We were able to fractionate the particles, both UHMWPE and Ti into the predetermined size ranges (Fig 1). Though the particles were obtained from commercial vendors, none-theless their size and shape were similar to those isolated from periprosthetic tissues by previous investigators[1,5,7] . Phagocytosis: Particles were phagocytosed in a size- and time-dependent manner. After $24 \mathrm{~h}$, all cells were observed to have phagocytosed between 1 and 30 particles, depending on particle size; more of the small particles were phagocytosed per cell than the large particles. Incubation 
beyond $24 \mathrm{~h}$ did not affect significantly the number of phagocytosed particles.

Membrane integrity: At $72 \mathrm{~h}$, membrane integrity was doseand size- dependently affected by particle treatment (Fig 2). At the highest dose, the micron-sized fraction had insignificant affect on membrane integrity, while the submicron- and nano-size fractions elicited the most robust negative response.
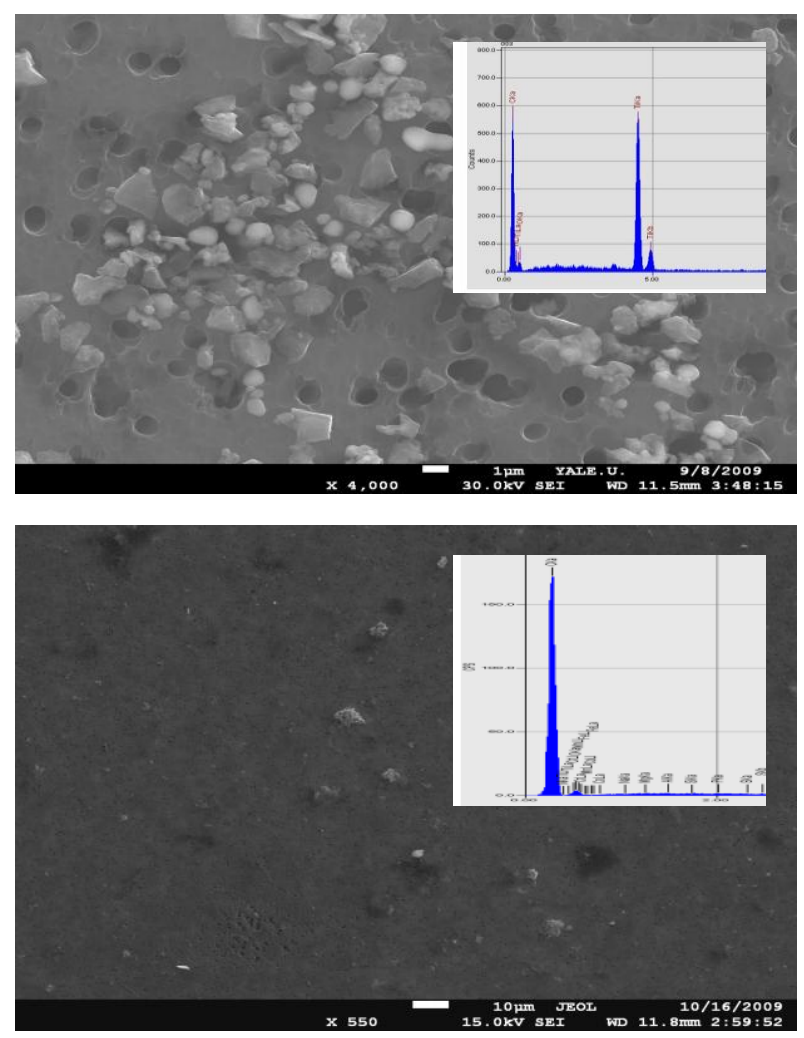

Figure 1: Representative SEM image of Ti particles (top panel) and UHMWPE particles (bottom panel) along with their EDF confirmations.

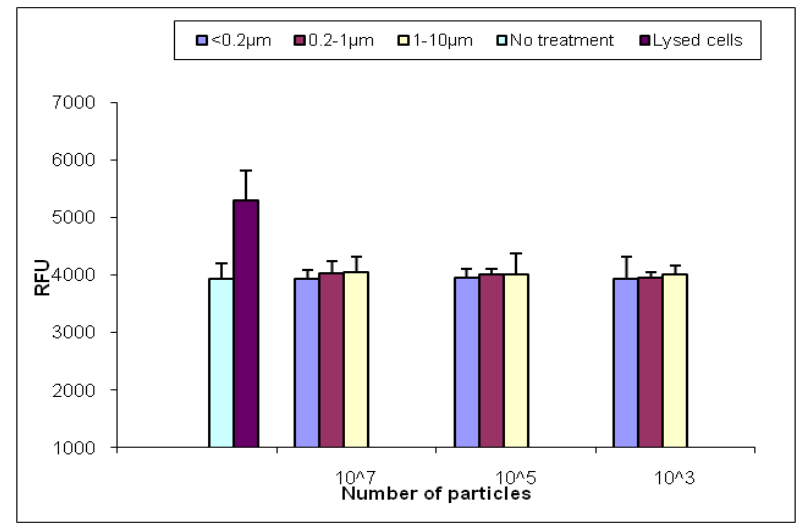

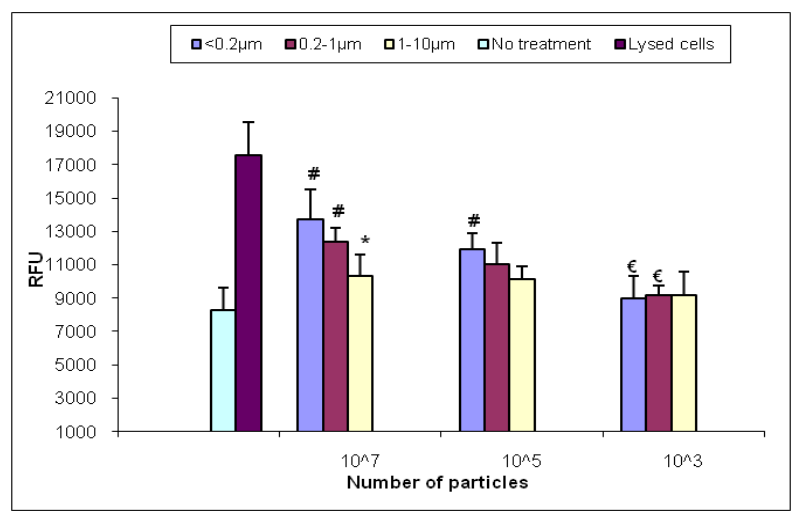

Figure 2: LDH release by RAW 264.7 cells at $24 \mathrm{~h}$ (top panel) and at $72 \mathrm{~h}$ in response to varying dose and sizes of both UHMWPE and Ti particles. All data are mean \pm SEM. \# $\mathrm{p} \leq 0.05$, significant difference between control and individual treatments; $* \mathrm{p} \leq$ 0.05 , significant difference between micron and nano- fractions at same dose; $€ \mathrm{p} \leq$ 0.05 , significant difference between $10^{7}$ dose and other doses of a particular size particle suspension

\section{CONCLUSIONS}

1. A technique has been developed for isolating UHMWPE particles reproducibly from simulated wear fluids and fractionating them into well defined size ranges.

2. RAW 264.7 cells phagocytosed greater numbers of nanoparticles than the larger particles. Further, membrane integrity was more negatively affected by the nano-sized particles than the larger particles, suggesting a more toxic effect of the smaller particles.

\section{REFERENCES}

1. Campbell P, Kossovsky N, Schmalzried TP. Particles in loose hips. J Bone Joint Surg Br 1993;75:161-162.

2. Endo M, Tipper JL, Barton DC, Stone MH, Ingham E, Fisher J. Comparison of wear, wear debris and functional biological activity of moderately crosslinked and non-crosslinked polyethylenes in hip prostheses. Proc Inst Mech Eng [H] 2002;216:111-122.

3. Fisher J, Bell J, Barbour PS, Tipper JL, Matthews JB, Besong AA, Stone $\mathrm{MH}$, Ingham E. A novel method for the prediction of functional biological activity of polyethylene wear debris. Proc Inst Mech Eng $[\mathrm{H}]$ 2001;215:127-132.

4. Goodman SB, Huie P, Song Y, Schurman D, Maloney W, Woolson S, Sibley R. Cellular profile and cytokine production at prosthetic interfaces. Study of tissues retrieved from revised hip and knee replacements. J Bone Joint Surg Br 1998;80:531-539.

5. Green TR, Fisher J, Stone M, Wroblewski BM, Ingham E. Polyethylene particles of a 'critical size' are necessary for the induction of cytokines by macrophages in vitro. Biomaterials 1998;19:2297-2302.

6. Jacobs JJ, Hallab NJ, Urban RM, Wimmer MA. Wear particles. J Bone Joint Surg Am 2006;88 Suppl 2:99-102.

7. McKellop HA, Campbell P, Park SH, Schmalzried TP, Grigoris P, Amstutz HC, Sarmiento A. The origin of submicron polyethylene wear debris in total hip arthroplasty. Clin Orthop Relat Res 1995;(311):3-20.

8. Shanbhag AS, Jacobs JJ, Black J, Galante JO, Glant TT. Human monocyte response to particulate biomaterials generated in vivo and in vitro. J Orthop Res 1995; 13:792-801.

9. Suzuki Y, Nishiyama T, Hasuda K, Fujishiro T, Niikura T, Hayashi S, Hashimoto S, Kurosaka M. Effect of etidronate on COX-2 expression and PGE(2) production in macrophage-like RAW 264.7 cells stimulated by titanium particles. J Orthop Sci 2007;12:568-577.

10. Bi Y, Collier TO, Goldberg VM, Anderson JM, Greenfield EM. Adherent endotoxin mediates biological responses of titanium particles without stimulating their phagocytosis. J Orthop Res 2002;20:696-703. 


\title{
Anterior Cruciate Ligament Fibroblast Response to Changes in Polymer Nanofiber Scaffold Composition
}

\author{
S.D. Subramony, M.S. Tracey, C. Erisken, M.Y. Elasmai, and H.H. Lu \\ Department of Biomedical Engineering, Columbia University, New York, NY
}

\begin{abstract}
Fibroblasts cultured on both PCL and PLGA nanofibers showed differing gene expression profiles. These findings indicate that cellular response is dependent on the mechanical properties of the underlying substrate.
\end{abstract}

\section{INTRODUCTION}

Ruptures and tears of soft tissues are among the most commonly reported injuries in the United States. Upwards of 100,000 anterior cruciate ligament (ACL) reconstructions are performed each year[1], with repair procedures involving grafting, implantation, or suturing of partially torn tissue[2]. Polymeric nanofibrous scaffolds have been suggested as a means of enabling functional ACL repair [3]. In particular, scaffolds fabricated from polylactide-coglycolide (PLGA) and poly- $\varepsilon$-caprolactone (PCL) have been evaluated for a variety of orthopaedic applications [4]. Although the biocompatibility of these polymers has been previously evaluated, it is currently unknown how the composition-dependent mechanical properties of these scaffolds (Table 1) affect cellular response. Therefore, in order to design an optimal ACL scaffold, the objective of this study was to evaluate the biosynthesis, proliferation, and gene expression of ACL fibroblasts when cultured on both PLGA and PCL nanofibers. It was hypothesized that the nanofiber matrix will support ligament fibroblast growth and differentiation, while substrate composition and mechanical properties will affect cellular response.

\section{METHODS}

\section{A. Scaffold Fabrication and Cell Culture}

Aligned PLGA (85:15, Lakeshore) and PCL (Lakeshore) nanofiber scaffolds were fabricated via electrospinning [4]. Bovine ACL fibroblasts were seeded on nanofiber scaffolds at a density of 30,000 cells $/ \mathrm{cm}^{2}$, and cell response was analyzed after $1,7,14$, and 28 days of culture.

TABLE 1

Polymer and Tissue Mechanical Properties [5]

\begin{tabular}{lllll} 
& $\begin{array}{l}\text { Fiber } \\
\text { Diameter }(\mathrm{nm})\end{array}$ & $\begin{array}{l}\text { Elastic Modulus } \\
(\mathrm{MPa})\end{array}$ & $\begin{array}{l}\text { Yield Strength } \\
(\mathrm{MPa})\end{array}$ & $\begin{array}{l}\text { Ultimate } \\
\text { Stress }(\mathrm{MPa})\end{array}$ \\
\hline hACL & 45 & 180 & 46.7 & 75.8 \\
PLGA & $615 \pm 152$ & $341 \pm 30$ & $9.8 \pm 1.1$ & $12.0 \pm 1.5$ \\
PCL & $700 \pm 140$ & $131 \pm 13$ & $9.1 \pm 0.6$ & $21.5 \pm 1.1$ \\
\hline
\end{tabular}

\section{B. End-Point Analysis}

Cellular attachment and morphology were assessed using the Live/Dead cell viability/toxicity assay. Total DNA $(n=5)$ was determined by the Picogreen DNA assay, while collagen synthesis $(n=5)$ was measured via the Sircol assay. Collagen distribution was also visualized by Picrosirius Red staining $(\mathrm{n}=2)$. The expression $(\mathrm{n}=3)$ of ligament fibroblast markers (collagen I, III, fibronectin, and tenascin C) was determined by RT-PCR. Gene expression was then normalized by the housekeeping gene GAPDH.

\section{Statistical Analysis}

ANOVA and the Tukey-Kramer post-hoc test were used for all pair-wise comparisons $\left({ }^{*} \mathrm{p}<.05\right)$.

\section{RESULTS}

\section{A. Cell attachment and Proliferation}

Fibroblasts cultured on both PCL and PLGA scaffolds proliferated throughout the 28-day study. Quantification of DNA (Fig. 1) revealed a steady increase in the number of cells over the course of the culture period, with significant increases between time points up to day 14 on the PLGA scaffolds and up until day 28 for the PCL group. Cells grown on PCL scaffolds also measured significantly higher cell number at day 28 , when compared to the relative plateau in growth exhibited by cells grown on PLGA. Their attachment morphology was visualized using confocal microscopy, in which cells were shown to assume a phenotypic elongated morphology, and were aligned along the long axis of nanofibers for both the PCL and PLGA groups.

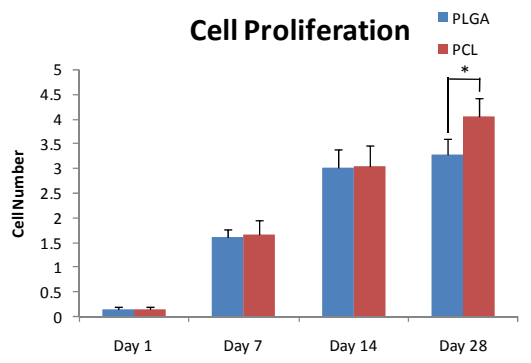

Fig 1. Cell proliferation on PCL and PLGA Scaffolds 


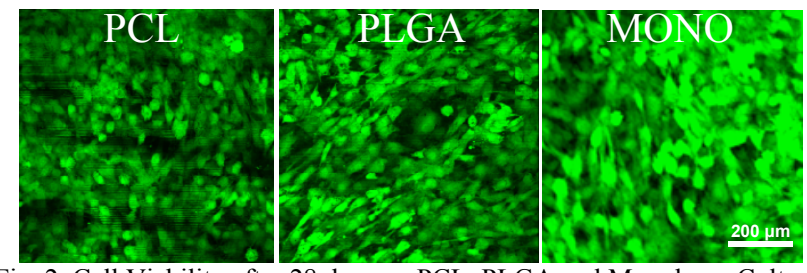

Fig. 2. Cell Viability after 28 days on PCL, PLGA and Monolayer Culture

In contrast, cells cultured in monolayer exhibited no preferential orientation and assumed a polygonal morphology (Fig. 2). The quantitative collagen assay indicated that a similar level of collagen matrix was produced on both polymer types with a more uniform surface morphology evident on the PLGA scaffolds, as compared to the undulating surface noticeable on the relatively less stiff PCL scaffolds (Fig. 3).

\section{B. Maintenance of Fibroblast Phenotype}

Gene expression of various fibroblast related gene markers was studied over time (Fig. 4). No significant change in fibronectin or tenascin $\mathrm{C}$ expression was observed, with relatively stable expression maintained from day 1 of culture. Cells cultured on the PLGA scaffolds showed a significant increase in type I collagen expression as compared to those cultured on PCL scaffolds by day 28 . Additionally, collagen III expression of cells cultured on PCL scaffolds was shown to be significantly higher than those cultured on PLGA scaffolds by day 28 .

\section{DISCUSSION}

In this study, significant differences in cell growth and gene expression were detected on PLGA and PCL scaffolds. Specifically, cell proliferation on the PCL based scaffolds was significantly greater than on the PLGA based scaffolds after four weeks in vitro. This behavior is most likely the result of the difference in degradation rate between PLGA, which degrades more rapidly via bulk erosion, and PCL, which degrades more slowly via surface erosion. Our results also suggest that differences in scaffold mechanical properties regulate the expression of key extracellular matrix components. Specifically, collagen type I expression was down-regulated while collagen type III expression was up-regulated by fibroblasts after 28 days of culture on PCL scaffolds.
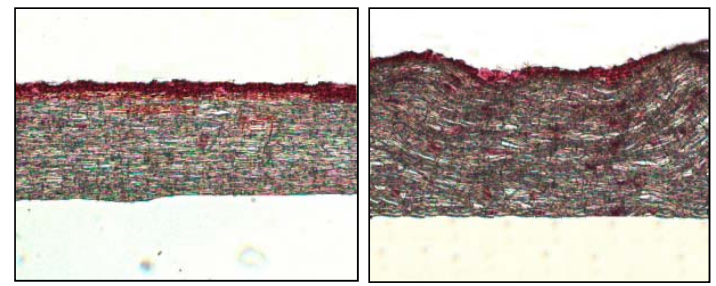

Fig. 3. Collagen deposition after 28 days of culture on PLGA and PCL (Picrosirius Red, 32X)

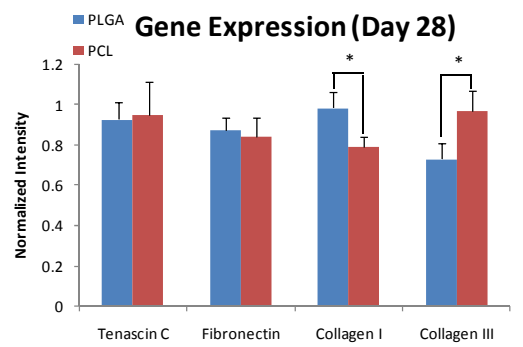

Fig. 4. Gene expression of key fibroblast markers $(* \mathrm{p}<.05)$.

Studies examining the injury response of fibroblasts in tendons and ligaments have shown similar trends in collagen production and regulation, indicating that the PCL culture environment may direct the fibroblasts towards granulation, and eventually scar tissue, formation [6]. The mechanical properties of PCL are lower than both those of PLGA scaffold and the native ACL (Table 1), and therefore may elicit a wound healing response as opposed to promoting native tissue formation. These findings indicate that substrate mechanics and composition influence fibroblast response. Future studies will aim to further characterize these polymer nanofibers and decouple the effect of polymer composition and mechanical properties in order to optimize the nanofiber-based scaffold system for ligament tissue engineering.

\section{ACKNOWLEDGEMENTS}

Funding from NIH/NIAMS (AR056459-02, AR055280-02), Wallace H. Coulter Foundation, Columbia Summer Undergraduate Research Fellowship (MST), National Science Foundation GK-12 Fellowship (SDS).

\section{REFERENCES}

[1] RS Gotlin, G Huie. "Anterior cruciate ligament injuries. Operative and rehabilitative options." Phys Med Rehabil Clin N Am, v11, pp895-928. 2000.

[2] M Kurosaka, S Yoshiya, JT Andrish. "A biomechanical comparison of different surgical techniques of graft fixation in anterior cruciate ligament reconstruction." Am J Sports Med, v15, pp225-229. 1987.

[3] R Vasita, DS Katti. "Nanofibers and their applications in tissue engineering." Int J Nanomedicine. v1,pp15-30. 2006.

[4] KL Moffat, JP Spalazzi, SB Doty, WN Levine, HH Lu. "Novel nanofiber based scaffold for rotator cuff repair and augmentation." Tissue Engineering, v15(1), pp115126. 2009.

[5] Z Ge, F Yang, JCH Goh, S Ramakrishna, EH Lee. "Biomaterials and scaffolds for ligament tissue engineering." J. Biomed. Mat. Res. A., v77A (3), pp639-652. 2006.

[6] TW Lin, L Cardenas, LJ Soslowsky. "Biomechanics of tendon injury and repair." J. Biomech.,v37 (6), pp865877. 2004. 


\title{
Quantifying and Understanding Protein Adsorption to Non-Fouling Surfaces
}

\author{
Ashutosh Agarwal $^{1}$, Parag Katira ${ }^{2}$, Henry Hess ${ }^{1}$ \\ ${ }^{1}$ Department of Biomedical Engineering, Columbia University, New York, NY \\ ${ }^{2}$ Department of Chemical Engineering, University of Texas at Austin, Austin, TX
}

\begin{abstract}
Surfaces grafted with poly(ethylene oxide) (PEO) are known to resist protein adsorption. Research efforts in this field have focused on both developing surfaces with better resistance to protein adsorption and understanding the origin of resistance of PEO grafted surfaces to protein adsorption. In the first part of this contribution, we describe a novel quantification technique for extremely low protein coverage on surfaces. This technique utilizes measurement of the landing rate of microtubule filaments on kinesin proteins adsorbed on a surface to determine the kinesin density. The detection limit of our technique is 100 times lower than that of standard characterization methods and is employed to test the performance of novel and established coatings with outstanding resistance to protein adsorption. In the second part, a random sequential adsorption (RSA) model is presented for protein adsorption to PEO coated surfaces. The model suggests that PEO chains act as almost perfect steric barriers to protein adsorption. Furthermore, it can be used to predict the performance of a variety of systems towards resisting protein adsorption and can help in the design of better nonfouling surface coatings.
\end{abstract}

\section{INTRODUCTION}

Protein-resisting surfaces are a crucial constituent in biomaterials and bionanotechnological applications (patterned surfaces for biosensors, investigation of cell behaviour, tissue engineering, etc.). The most widely used method to render a surface protein resistant (non-fouling) is surface immobilization of poly(ethylene oxide) (PEO) chains of varying lengths (number of EO units varying from 3 to 50).

The best performing coatings have shown to reduce protein adsorption to less than $0.1 \%$ of a monolayer [1]. For an average protein, this represents a surface density of approximately $1 \mathrm{ng} / \mathrm{cm}^{2}$. This low coverage of proteins on surfaces is at or below the limit of detection for standard protein density quantification techniques [1]. Consequently, there is a critical need for a new characterization technique to test and compare state-of-the-art surface coatings. We introduce a characterization technique which achieves a detection limit for the presence of kinesin motor proteins of $0.1 \mu \mathrm{m}^{-2}\left(\sim 0.004 \mathrm{ng} / \mathrm{cm}^{2}\right)$ [2]. In this method, the landing rate of fluorescently labeled microtubule filaments is measured to calculate the surface densities of motor proteins adsorbed to a test coating surface. The simplicity of design and ease of detection using an epi-fluorescence microscope make this technique useful for characterization of a large variety of protein resistant surfaces.
Understanding the mechanism of the resistance to protein adsorption by PEO chains and modeling the effect of PEO characteristics such as chain length and terminating group on protein adsorption is the key towards designing high performance polymer coatings. While earlier models treated PEO chains as steric barriers that prevented the proteins from interacting with the surface [3], the contemporary focus of modeling is towards employing molecular level interactions between polymers, polymers and proteins, the protein and the surface, polymers and the surface, and polymers and solvent molecules [4]. However, in all these treatments, polymer chains are assumed to be evenly distributed across the surface, with a constant spacing determined by the grafting density. We hypothesize that the distribution of the polymer chains is close to random and this leads to stochastically distributed protein binding sites defined by the absence of polymer chains. A Random Sequential Adsorption (RSA) model is presented which focuses on the random distribution of polymer chains on the surface leading to polymer-free "bald" spots [5].

\section{RESULTS AND DISCUSSION}

\section{A. Landing Rate Methodology}

Microtubule attachment rate measurements are interpreted in the context of a two stage adsorption model: First, kinesin molecules adsorb irreversibly from solution to the surface, filling a fraction of the available binding sites. Second, the kinesin solution is replaced by a microtubule solution and microtubules adsorb specifically to the kinesin.

The surface concentration of adsorbed kinesin motors obtained for a given dosage of kinesin solution is quantified by measuring the initial landing rate $\mathrm{R}$ of microtubules on the coated surfaces, and the maximal, diffusion-limited landing rate $\mathrm{Z}$ on a control surface densely coated with motor proteins [6]. The probability of microtubule landing and binding to the surface is equal to the probability that there is at least one kinesin motor in the area $\mathrm{A}$ of interaction between the microtubule and the surface adsorbed kinesin. The probability distribution of kinesin molecules in an area on the surface can be approximated by a Poisson distribution. Therefore, one can express the initial landing rate of microtubules $\mathrm{R}$ as a function of the kinesin surface density $\rho$, the diffusion limited landing rate $\mathrm{Z}$ and the interaction area $\mathrm{A}$ as:

$$
R=Z *[1-\exp (-\rho A)]
$$




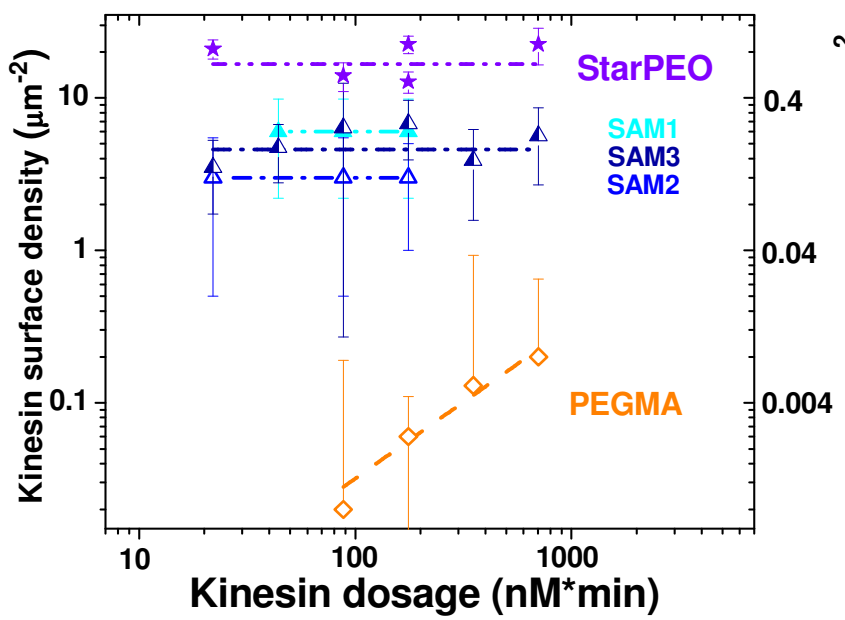

Fig. 1: Kinesin surface density determined on non-fouling surfaces as a function of kinesin dosage.

The initial landing rate $\mathrm{R}$ begins to substantially deviate from the diffusion-limited maximal landing rate if $\rho \mathrm{A}<1$, where $A \sim 0.1 \mu \mathrm{m}^{2}$ for typical microtubule lengths. Since the minimal measurable landing rate is on the order of $1 \mathrm{~mm}^{-2} \mathrm{~s}^{-1}$, and the diffusion limited landing rate $\mathrm{Z}$ is on the order of 100 $\mathrm{mm}^{-2} \mathrm{~s}^{-1}$, microtubule landing rate measurements are sensitive

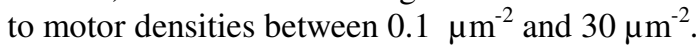

\section{B. RSA model for protein adsorption}

Grafted PEO polymers and preadsorbed proteins are modeled as randomly distributed, pre-existing obstacles on the surface. The density of adsorbed proteins on the surface, $\sigma$ is given by:

$\sigma=\frac{4 f(1-x)}{\pi d_{p}^{2}}\left\{1-\left[1+\frac{\pi d_{p}^{2}(4 f-1) S Z t}{4 f(1-x)}(1-x)^{2 \rho_{0} \pi d_{p} R_{g}}\right]^{-1 /(4 f-1)}\right\}$

In this equation, the input parameters for the protein are collision rate, $\mathrm{Z}$, mass of protein, $\mathrm{m}_{\mathrm{p}}$, concentration, $\mathrm{C}$, diameter of protein, $d_{p}$, packing fraction of proteins, $f$, sticking probability, $\mathrm{S}$, time for adsorption, $\mathrm{t}$ and the input parameters of the polymer are radius of gyration, $R_{g}$, degree of polymerization, $\mathrm{N}$, maximum grafting density, $\rho_{0}$, and grafting ratio, $x$.

Due to the small number of parameters, the predictions of the above model can be directly compared to published experimental results. The correspondence between the experimental data and the theoretical predictions despite the complete neglect of any thermodynamic and structural detail is striking.

\section{CONCLUSIONS}

Our landing rate method extends the lower end of the detection range of established methods. In essence, microtubule landing rate measurements afford single molecule sensitivity by exploiting the thousand-fold amplification of a fluorescence signal provided by labeled microtubules. In the present form, this method is limited to measurement of kinesin surface densities and cannot be used to estimate fouling by
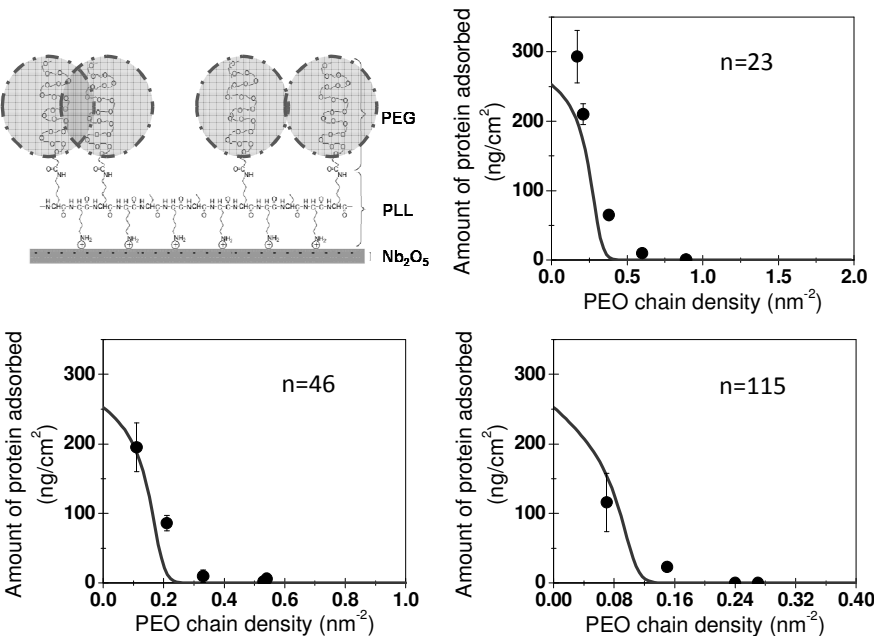

Fig. 2: Comparison with experimental data of [7]. The adsorption of serum protein to PEG-grafted surfaces as function of PEO chain density as experimentally observed (black circles) and as predicted by the RSA model (solid line).

blood proteins, for example. However, that does not limit the method's applicability in the determination of relative kinesin motor density on different surfaces to optimize and standardize the coating manufacturing process.

The implications of the proposed RSA model for the longstanding debate about the origin of the resistance of PEOgrafted surfaces to protein adsorption are profound. The model suggests that the PEO coating presents neither a thermodynamic nor a kinetic barrier which is surmounted over time, but a perfect steric barrier with a defined number of holes. The key suggestion for the design of coating procedures is to increase the PEO chain density to reduce residual protein adsorption.

\section{REFERENCES}

[1] A. Hucknall, S. Rangarajan, and A. Chilkoti, "In Pursuit of Zero: Polymer Brushes that Resist the Adsorption of Proteins," Advanced Materials, vol. 21, no. 23, pp. 2441-2446, 2009.

[2] P. Katira, A. Agarwal, T. Fischer et al., "Quantifying the performance of protein-resisting surfaces at ultra-low protein coverages using kinesin motor proteins as probes," Advanced Materials, vol. 19, pp. 3171-3176, 2007.

[3] S. I. Jeon, L. H. Lee, J. D. Andrade et al., J. Colloid Interface Sci., vol. 142, pp. 149-158, 1991.

[4] J. Satulovsky, M. A. Carignano, and I. Szleifer, "Kinetic and thermodynamic control of protein adsorption," Proceedings of the National Academy of Sciences of the United States of America, vol. 97, no. 16, pp. 9037-9041, Aug, 2000.

[5] P. Katira, A. Agarwal, and H. Hess, "A Random Sequential Adsorption Model for Protein Adsorption to Surfaces Functionalized with Poly(ethylene oxide)," Advanced Materials, vol. 21, no. 16, pp. 15991604, 2009.

[6] J. Howard, A. J. Hudspeth, and R. D. Vale, "Movement of microtubules by single kinesin molecules," Nature, vol. 342, pp. 154-158, 1989.

[7] S. Pasche, S. M. De Paul, J. Voros et al., "Poly(1-lysine)-graftpoly(ethylene glycol) Assembled Monolayers on Niobium Oxide Surfaces: A Quantitative Study of the Influence of Polymer Interfacial Architecture on Resistance to Protein Adsorption by ToF-SIMS and in Situ OWLS," Langmuir, vol. 19, no. 22, pp. 9216-9225, 2003. 


\title{
Neural Differentiation of Human Neural Stem/Progenitor Cells on Piezoelectric Scaffolds
}

\author{
Y-S. Lee, G. Collins, T. Livingston Arinzeh \\ New Jersey Institute of Technology, Newark, NJ 07102
}

\begin{abstract}
This study evaluated the neural differentiation of human neural stem /progenitor cells (hNPCs) on electrospun fibrous piezoelectric polyvinylidene fluoride-trifluoroethylene (PVDF-TrFE) scaffolds. We hypothesize aligned fibrous piezoelectric scaffold would provide both physical cues (via contact guidance) and local electrical activity to promote neuronal differentiation and neurite extension. hNPCs predominately differentiated into the neuronal lineage on both random and aligned electrospun PVDF-TrFE scaffolds. Less nestin-positive hNPCs were present on the scaffolds compared to laminin coated surface. The results suggest the potential use of these scaffolds in spinal cord repair and other neural applications.
\end{abstract}

\section{INTRODUCTION}

Electric fields can influence neural growth and orientation in vitro [1] and have been applied for the treatment of spinal cord injuries in recent clinical trials [2]. A piezoelectric polymer, poly vinyldifluoride-trifluropolyethylene (PVDF-TrFE) can induce a transient surface charge in the absence of additional energy sources or electrodes. Unlike homopolymer PVDF, the steric hindrance of TrFE forces the PVDF into an all-trans crystal configuration, thus, rendering the polymer piezoelectric [3]. The piezoelectric properties of PVDF-TrFE were further enhanced by annealing to increase crystal organization. Electrospinning is a common method in tissue engineering applications to produce continuous fibers with high surface area to volume ratio. Random, aligned, and patterned nano-fibrous mesh and three-dimensional structures can be fabricated by altering collection methods. The topographic features of nano-aligned-fibrous scaffolds create contact guidance, which may further facilitate axonal extension. Human neural stem/progenitor cells (hNPCs) have the ability to differentiate into astrocyte, oligodendrocytes, and neurons [4]. This study investigated the neural differentiation of hNPCs on fibrous, PVDF-TrFE to determine its potential use as a scaffold in nerve repair. Comparisons were made with laminin coated tissue culture plastic (control).

\section{Methods}

\section{A. Scaffold Fabrication}

Polymer solutions for electrospinning were prepared with PVDF-TrFE and methyl-ethyl-ketone (MEK) as the solvent. Random and aligned electrospun scaffolds were collected on a stationary plate and a rotating drum, respectively. Annealed samples were kept at $135^{\circ} \mathrm{C}$ for 96 hours and quenched with ice water. All scaffolds were vacuum dried for 48 hours prior material characterization and in vitro cell study.

\section{B. Material Characterization}

Scanning electron microscopy (SEM) images were taken to evaluate the fiber diameter and orientation. Differential scanning calorimetry (DSC) was used to evaluate thermally active transition such as melting temperature. X-ray diffraction (XRD) was performed to evaluate crystal structure of as-spun and annealed PVDF-TrFE scaffolds. Thermally-stimulated current (TSC) was used to confirm piezoelectricity by measuring the current indicating the dipole movement in response to an increase in temperature.

\section{In vitro study}

hNPCs were purchased from Lonza, which are cryopreserved neurospheres obtained from 20 weeks-old fetal brain tissue. They were seeded at 45,000 cells $/ \mathrm{cm}^{2}$ and cultured in differentiation media (Lonza) with $25 \mathrm{ng} / \mathrm{mL}$ brain-derived nerve growth factor (BDNF, R\&D Systems) or standard growth media for 9 days. Comparisons were made with laminin coated plates. The cells were fixed and stained with antiNestin, glial fibrillary acidic protein (GFAP), and neuron-specific beta-III tubulin to identify hNPCs, astrocytes, and neuron, respectively. DAPI was also applied as counter stain. 4 images were taken for each sample ( $n=6$ per group) and positive stain was manually counted to obtain percentage of differentiation. One-way analysis of variance (ANOVA) and Tukey-Kramer test were used to determined the statistic significance between the groups $(\mathrm{p}<0.05)$.

\section{Results}

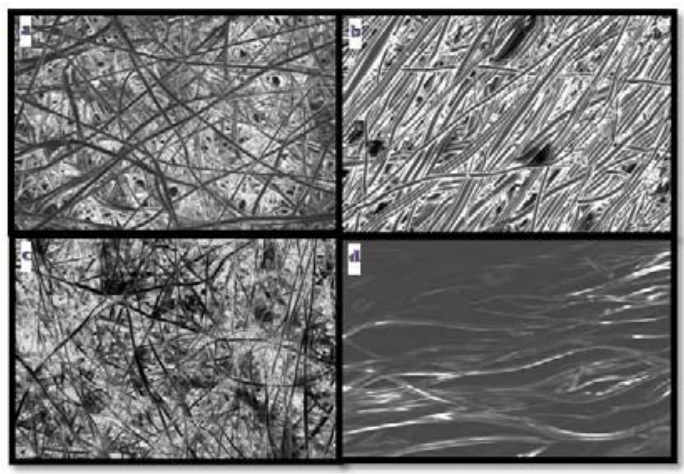

Figure 1. SEM image of random (left) and aligned (right) electrospun small (a,b) (3500x) and large(c,d) (1500x) PVDF-TrFE fibrous mesh. 
The average fiber diameter of micron- (L) and sub-micron- (S) PVDF-TrFE were $3.32 \pm 0.2 \mu \mathrm{m}$ and $0.75 \pm 0.08 \mu \mathrm{m}$, respectively (figure 1). The melting point of as-spun of PVDF-TrFE (L) and (S) increased from $147.9^{\circ} \mathrm{C}$ and $147.8^{\circ} \mathrm{C}$ to $152.4^{\circ} \mathrm{C}$ and $154.5^{\circ} \mathrm{C}$ after annealing, respectively. The increase in melting temperature suggested an increase in crystallinity due to annealing. XRD results indicated an increase in the intensity of the piezoelectric beta phase at $20.4^{\circ}$ and the loss of the non- piezoelectric alpha phase around $18.5^{\circ}$ on the annealed in comparison to the as-spun samples (figure 2). The annealing process induced crystal organization hence, enhancing the piezoelectric properties.
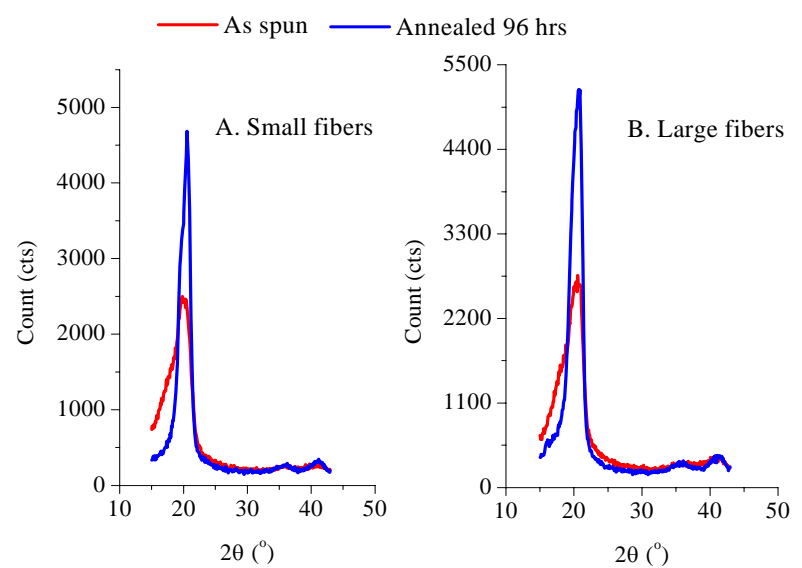

Figure 2. XRD results of electrospun small (a) and large (b) as spun and annealed PVDF-TrFE scaffolds.
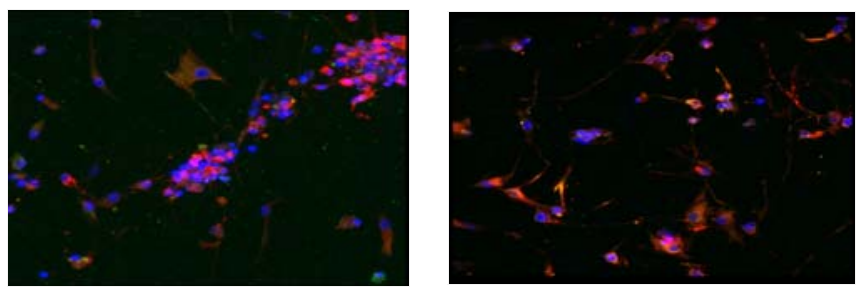

(a)

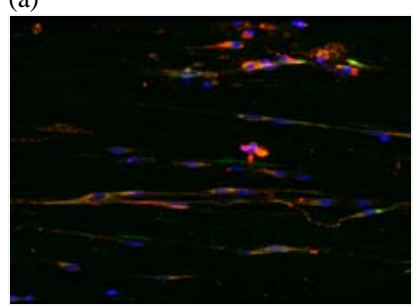

(c)
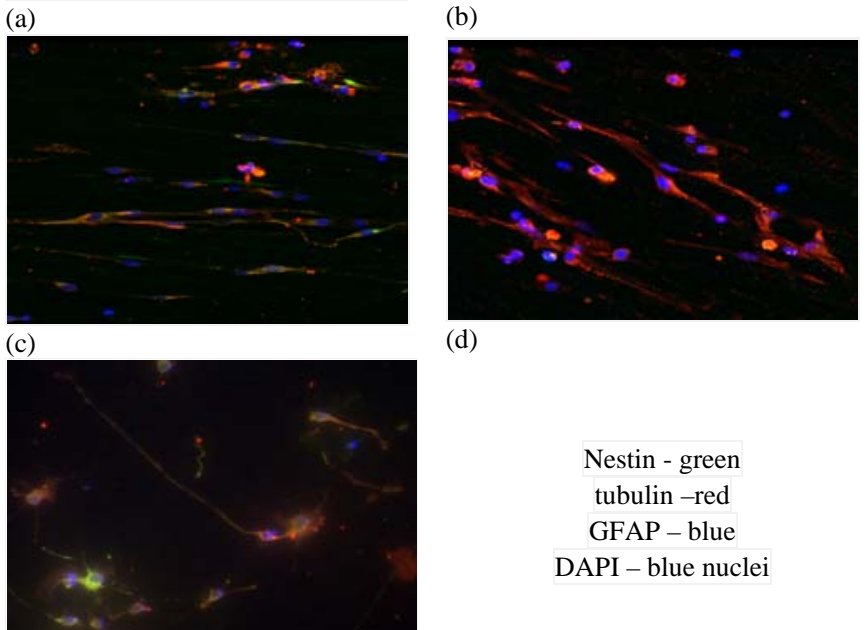

(d)

$$
\begin{aligned}
& \text { Nestin - green } \\
& \text { tubulin -red } \\
& \text { GFAP - blue } \\
& \text { DAPI - blue nuclei }
\end{aligned}
$$

(e)

Figure 3. Confocal microscopy images of hNPCs on PVDF-TrFE-L a) random, b) random-annealed, c) aligned, d) aligned-annealed (all at 40x obj), and e) laminin (20x obj.) in induction media.
hNPCs have differentiated predominately along the neuronal lineage indicated by the beta-tubulin positive stains on the scaffold and the laminin coated plates in the induction media (figure 3). Less nestin-positive hNPCs were observed on the scaffolds than on the laminin (figure 4). In the control media, hNPC on the laminin showed more nestin-positive cells compared to the scaffolds (data not shown).

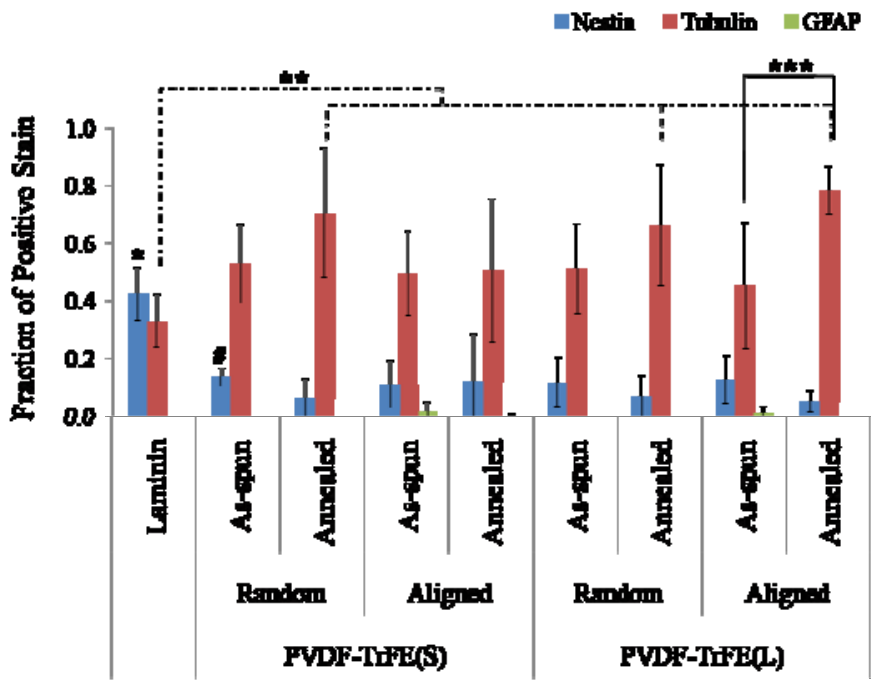

Figure 4. Fraction of positive nestin, tubulin, and GFAP on various scaffolds in induction media. ${ }^{*}$ Nestin positive cells were the highest on laminin. **Tubulin positive cells were the lowest on laminin in comparison to annealed, random and aligned PVDF-TrFE(L) and annealed random PVDF-TrFE(S). ***Tublin positive cells on annealed, aligned $\mathrm{PVDF}-\operatorname{TrFE}(\mathrm{L})$ was significantly greater than as-spun aligned PVDF-TrFE(L). Very little GFAP positive cells could be detected in all of the samples.

\section{Discussion}

This study demonstrated the potential use of this scaffold for nervous tissue repair. The scaffolds enhanced neural differentiation, as indicated by a lower level of nestin positive cells on scaffolds in comparison to laminin surfaces. Neuronal differentiation may be enhanced on annealed scaffolds, which display higher piezoelectricity, as indicated by the higher fraction of cells expressing beta-III tubulin.

\section{AcKNOWLEDGEMENT}

The authors thank funding sources from the New Jersey Commission on Spinal Cord Research.

\section{REFERENCES}

[[1] N.B. Patel, M.M. Poo, "Perturbation of the direction of neurite growth by pulsed and focal electric fields,” J Neurosci, vol 4, pp 2939-47, 1984.

[[2] L.D. Duffell LD, "Long-term intensive electrically stimulated cycling by spinal cord-injured people: Effect on muscle properties and their relation to power output," Muscle Nerve, vol 38, pp 1304-11, 2008.

[[3] A.J. Lovinger, "Ferroelectric Polymers,” Science, vol 220, pp 1115-21, 1983.

[[4] F.H. Gage, "Mammalian Neural stem Cells," Science, vol 287, pp 1433-8, 2000 


\title{
Decreased lung carcinoma cell density on select polymer nanometer surface features for lung replacement therapies
}

\author{
Lijuan Zhang ${ }^{1}$ and Thomas J. Webster ${ }^{2}$ \\ ${ }^{1}$ Department of Chemistry and ${ }^{2}$ Division of Engineering \\ Brown University \\ Providence, RI 02912
}

\begin{abstract}
PLGA (poly-lactic-co-glycolic acid) has been widely used as a biomaterial in regenerative medicine due to its biocompatibility and biodegradability. The purpose of the present in vitro research was to prepare PLGA films with various nanometer surface features and determine whether lung cancer epithelial cells respond differently to such topographies. Different size polystyrene beads were used to cast poly(dimethylsiloxane) (PDMS) molds which were used as templates to create nano-featured PLGA films. Atomic force microscopy (AFM) images and root mean square (RMS) roughness values indicated that the intended spherical surface nano-topographies on PLGA were formed. A solution evaporation method was also utilized to modify PLGA surface features by using 8 wt $\%$ and $4 \%$ chloroform solutions. Most importantly, lung cancer epithelial cells adhered less on the PLGA surfaces with RMS values of $0.62,2.23$ and $5.42 \mathrm{~nm}$ after 4 hours of culture compared to any other PLGA surface created here. After 3 days, PLGA surfaces with an RMS value of 0.62 nm had much lower cell density than any other sample. In this manner, PLGA with specific nanometer surface features may inhibit lung cancer cell density, providing an important biomaterial for the treatment of lung cancer with wide range of applications.
\end{abstract}

\section{INTRODUCTION}

The interaction between cells and extracellular matrix (ECM) proteins is complicated, drawing much attention for biomaterial interfacial research since understanding this interaction will significantly contribute to the design of better implants. Different implant material properties, including surface chemistry, surface texture and surface energy have been investigated to date to create better implantable materials. Specifically, recent research has demonstrated that implant nanometer morphology had greatly influenced cell attachment, proliferation and differentiation [1]. Various different micro- and nano-structured patterns on substrate surface have been created to investigate the cell responses. However, the underlying mechanism of cell response to micro- and nanometer patterns is still poorly understood. In this study, uniform nano-spherical surfaces were created on poly-lactic-co-glycolic acid (PLGA) films to evaluate lung carcinoma epithelial cell responses on such surfaces. Cell attachment and short-term cell density assays were examined on the novel nano-rough PLGA surfaces.

\section{MATERIALS AND METHODS}

\section{A. Fabrication of Nanometer Surface PLGA Films}

PLGA (poly-lactic-co-glycolic acid) films with nanometer spherical surface features were created using a method previously described by Carpenter et al [2]. Briefly, different size (specifically, $190 \mathrm{~nm}, 300 \mathrm{~nm}, 400 \mathrm{~nm}$, and $530 \mathrm{~nm}$ diameter) polystyrene beads were placed onto glass coverslips to create monolayers of highly ordered arrays. Then, poly(dimethylsiloxane) (PDMS) molds were prepared by placing PDMS onto these various polystyrene monolayers. The resulting PDMS molds were used as templates to cast PLGA films with various nanometer surface features. Finally, soluble PLGA was poured over the PDMS molds which were then allowed to evaporate at room temperature. As a result, the surface of the resulting PLGA films replicated the initial polystyrene nanobead topographical features. A solution evaporation method was also used to control PLGA surface roughness by using $8 \mathrm{wt} \%$ and $4 \%$ chloroform solutions.

\section{B. Surface Characterization}

Atomic force microscopy (AFM) images, electron spectroscopy for chemical analysis (ESCA) and contact angles were used to characterize the surfaces.

\section{Cell Culture and Density Experiments}

Lung carcinoma cells (A549; ATCC) were seeded at a density of either 3,500 cells $/ \mathrm{cm}^{2}$ or 7,000 cells $/ \mathrm{cm}^{2}$ and cultured in $\mathrm{F}-12 \mathrm{~K}$ medium with $10 \%$ fetal bovine serum (FBS) at $5 \% \mathrm{CO}_{2}$ and $37^{\circ} \mathrm{C}$ for $4 \mathrm{hrs}$. After $4 \mathrm{hrs}$, cells were stained and counted under a fluorescence microscope. Also, lung carcinoma cells were seeded at a density of 50,000 cells $/ \mathrm{cm}^{2}$ and cultured in $\mathrm{F}-12 \mathrm{~K}$ medium with $10 \%$ fetal bovine serum (FBS) at $5 \% \mathrm{CO}_{2}$ and $37^{\circ} \mathrm{C}$ for 3 days. After 3 days, cells were typsinized and counted. All experiments were run in duplicate and repeated at least three separate times. Results were analyzed for statistical significance using Student T-tests. 


\section{RESULTS AND DISCUSSION}

AFM images and root mean square roughness (RMS) values provided evidence that the intended spherical surface nano-topographies on PLGA with RMS values of 2.23, 5.03, 5.42 and $36.90 \mathrm{~nm}$ were formed for the PLGA surfaces which employed 190, 300, 400, and $530 \mathrm{~nm}$ beads. The solution evaporation method was also used to control PLGA surfaces with RMS values of 0.62 and $2.23 \mathrm{~nm}$ by using $8 \mathrm{wt} \%$ and 4 $\%$ PLGA chloroform solutions, respectively. ESCA results showed the same chemistry between all PLGA surfaces created by the two different methods (that is, using different concentrations of PLGA in chloroform or using PS beads).

Cell adhesion results showed the least number of lung carcinoma epithelial cells adherent to the PLGA surfaces with RMS values of $0.62,2.23$ and $5.42 \mathrm{~nm}$ compared to nanosmooth (currently-used) PLGA (Fig. 1). Importantly, the trend of decreasing cancerous cell density with decreased nanometer RMS values was not linear as PLGA surfaces with an RMS value of 5.42 showed nearly the same cell adhesion as PLGA surfaces with an RMS value of 0.62 . Such results suggest that nano-rough surfaces with specific RMS values may inhibit cancer cell functions by reducing their density. Three day cell proliferation results on the different nanorough PLGA surfaces revealed similar trends as the adhesion results (Fig. 2). Specifically, PLGA surfaces with an RMS value of $0.62 \mathrm{~nm}$ had much lower cell density. Interestingly, the $0.62 \mathrm{~nm}$ RMS PLGA was one of the most hydrophobic PLGA surfaces created in this study which may have influenced initial protein adsorption events to decrease cell density. Identification of altered initial protein adsorption events responsible for this trend needs to be the focus of future studies. In summary, results of this study demonstrated significantly less lung cancer epithelial cell density on PLGA surfaces with an RMS value of $0.62 \mathrm{~nm}$ which should be further explored to reduce cancer cell growth when developing lung replacements for lung cancer patients.
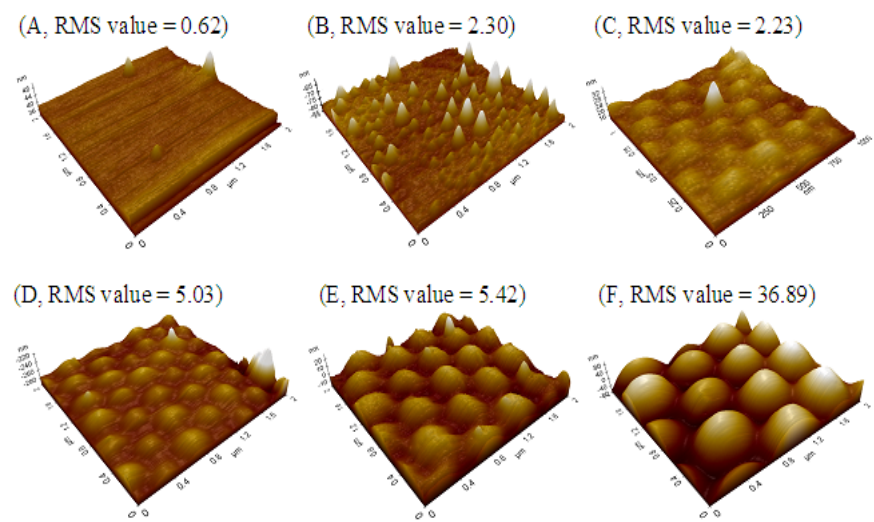

Fig. 1. AFM images and RMS values: (A-B) AFM images of PLGA surfaces created by solution evaporation with (A) $8 \%$ and (B) $4 \%$ chloroform solutions. (C-F) AFM images of PLGA surfaces created by using templates of PS nanobeads with a diameter of (C) $190 \mathrm{~nm}$, (D) $300 \mathrm{~nm}$, (E) $400 \mathrm{~nm}$ and (F) $530 \mathrm{~nm}$.

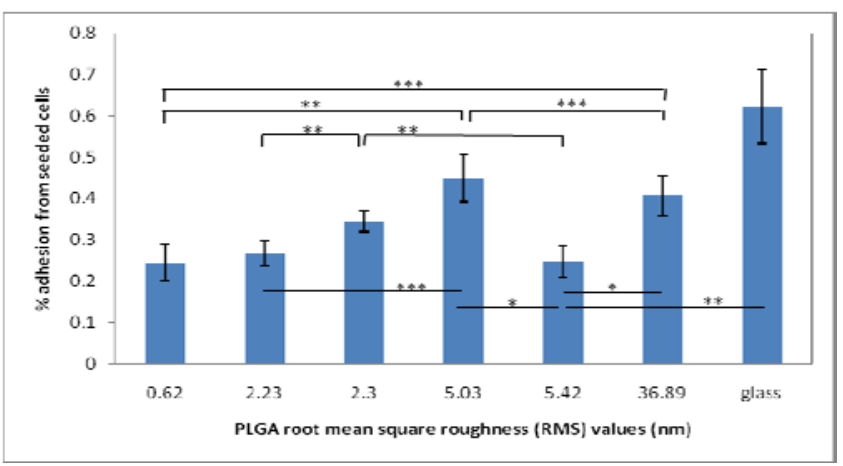

Fig. 2. Lung cancer epithelial cell adhesion results on the various PLGA samples and borosilicate glass after four hours. Data expressed as mean \pm standard deviation of the mean. * $\mathrm{p}$

$$
<0.01,{ }^{* *} \mathrm{p}<0.05 \text { and }{ }^{* * *} \mathrm{p}<0.1 \text {. }
$$

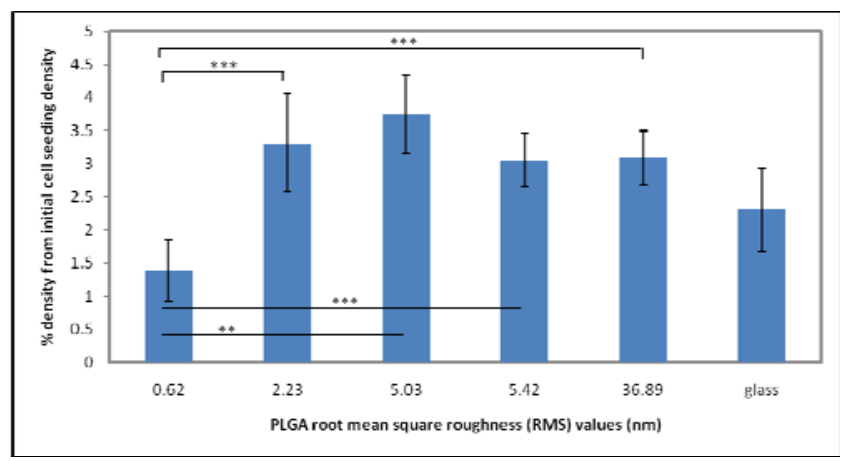

Fig. 3. Lung cancer epithelial cell density results on the various PLGA samples and borosilicate glass after three days. Data expressed as mean \pm standard deviation of the mean. ${ }^{* *}$ p

$$
<0.05 \text { and }{ }^{* * *} \mathrm{p}<0.1 \text {. }
$$

\section{CONCLUSIONS}

In this research, a simple method was used to create nanorough PLGA surfaces with highly ordered nanospherical structures. The results of lung epithelial cancerous cell density assays showed that PLGA surfaces with an RMS value of $0.62 \mathrm{~nm}$ (followed by PLGA surfaces with an RMS value of $5.42 \mathrm{~nm}$ ) inhibited lung epithelial cancerous cell densities the most. Since contact angles also significantly changed (but surface chemistry remained the same) among the various PLGA nanometer surfaces created, a surface energy related mechanism may be responsible. In summary, this study demonstrated that specific PLGA nanometer surface features should be further studied for lung replacement strategies that require decreased cancerous cell growth.

\section{ACKNOWLEDGEMENT}

The authors would like to thank the Hermann foundation for funding and Northeastern Research Center for AFM use.

\section{REFERENCES}

[1] H. Liu and T. J. Webster, "Nanomedicine for implants: A review of studies and necessary experimental tools," Biomaterials, vol. 28, pp. 354-369, Jan 2007.

[2] J. Carpenter, et al., "Nanometer polymer surface features: the influence on surface energy, protein adsorption and endothelial cell adhesion," Nanotechnology, vol. 19, p. 505103, Dec 172008. 


\title{
The Origin and Effect of Space Charges in Electrospinning
}

\author{
Rashi Grewal, Mike Jaffe, John Federici, Bryan Pfister and George Collins \\ Department of Biomedical Engineering \\ New Jersey Institute of Technology \\ Newark, NJ 07102
}

\section{I.Introduction}

This work involves the study of the origin and effect of space charges in the electrospinning process. Electrospinning is a process used to make solid, nanofibrous polymer mats. These mats are used in tissue engineering as scaffolds for cells. The origin and effect of space charges in this process has not been well-studied.

\section{II.Experimental}

A polymer solution is loaded into a plastic syringe capped by a needle. The lead of a wire from a high DC voltage supply is connected to the needle, from where the solution will flow out. The opposing lead is connected to a metal plate. A syringe pump hydraulic system creates pressure to push the solution out from its syringe. The polymer solution is drawn out of the syringe by the electric field created by the high voltage, and forms a Taylor cone at the tip of the needle. This Taylor cone is then elongated into fibers that travel towards the metal plate, where they are then collected. These fibers complete the circuit. The presence of residual charges was detected using thermally stimulated current (TSC). Novel results were obtained using a highly sensitive electrometer to measure current between the needle and grounded plate under different conditions.

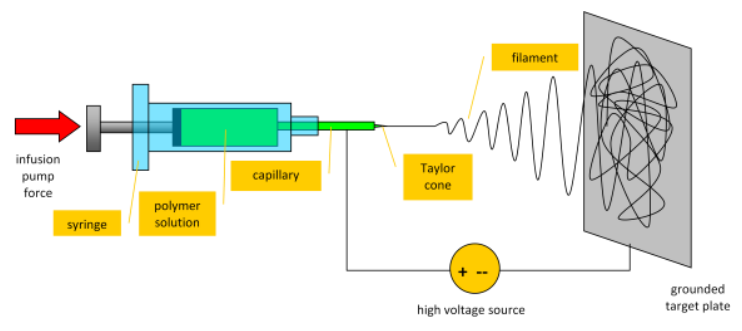

Fig. 1. Electrospinning Setup

\section{III.Initial Results and Discussion}

Electrospinning is possible because the charges that are injected into the polymer solution electrostatically drive the ejected fluid stream from the needle to the grounded plate. The focus here is on the fate of the charges in the resulting fibrous mat. It has been previously shown that residual charges can be detected in electrospun mats. These are space charges trapped within the internal structure of the solid. It was assumed that the singular origin of these charges was injection into the solution as it exited the metal needle. Space charges collect at irregularities such as defects or grain boundaries, and on the surface at the dielectric-electrode interface [1-3].

It is possible to remove space charges from their locations within the polymer. The decay, or release, of space charges occurs through either neutralization with atmospheric charges or dissipation from the surface or bulk of the material. There are various techniques to dissipate space charges: UV irradiation, and water or alcohol soaking. In the case of UV irradiation, the polymer sample is exposed to UV radiation source. The energy of the photons is greater than that of the space charges, which excites the charges and causes them to become detrapped, or mobile, from their locations and released into the air. The presence of space charges has also been observed to be greatly reduced when a polymer is soaked in water or alcohol. The actual mechanism behind which this works has yet to be understood. [46].

The role of space charges in electrospun scaffolds has not been studied. Preliminary results in Table I indicate that the total charge of a mat treated to dissipate charge was less than a freshly electrospun untreated mat. One aim of this study is to examine the effect of space charges on protein adsorption by comparing freshly electrospun mats treated to dissipate the charge. 
Table I

Total Current of Samples

\begin{tabular}{|c|c|}
\hline Sample & Total Current \\
\hline Untreated & $2.67807 \mathrm{E}-7$ \\
\hline UV & $9.37262 \mathrm{E}-9$ \\
\hline Water & $1.37646 \mathrm{E}-7$ \\
\hline Ethanol & $7.13985 \mathrm{E}-8$ \\
\hline
\end{tabular}

If the electric field strength at the tip of the needle, which is an electrode, surpasses the ionization threshold of the surrounding atmosphere, this creates a local ionized region, in which the positive and negative charges of the surrounding atmosphere become separated. The ions of the same charge as the voltage supply are repelled and travel towards the opposite electrode. This invites the possibility that there are two sources of space charges: those that are directly injected into the polymer solution, and those that are deposited by the corona [7-10].

By measuring the current in the absence of a polymer solution in the syringe, it was determined that a corona current was indeed detected. Under normal electrospinning conditions, it was observed that the corona current is the first event to occur once the voltage supply is turned on. No previous study has examined the role of the corona on the electrospinning process. The corona may be influenced by electrospinning process parameters and environmental conditions such as humidity. Table II A shows the variation of the corona current with needle size and humidity. In a modified configuration, the plastic syringe was pierced with a needle so that the high field was generated inside the body of the syringe. For this case, current could not be detected on the microamp scale. This is shown in Table II B.

It has been recently reported that the Taylor cone is not only the site of ejection of the fluid stream, but also the site of corona discharge (7). Combined with the results of this study, it is suggested that conventional electrospinning always occurs in the presence of a corona, and that the space charges in the resulting electrospun mats originate from direct injection as well as from corona deposition.

Table II A

Typical Cofiguration vs. Corona Current

\begin{tabular}{|c|c|}
\hline Configuration & Current \\
\hline Lg. Needle & $1.98 \mu \mathrm{A}$ \\
\hline Lg. Needle w/ Humidifier & $1.66 \mu \mathrm{A}$ \\
\hline Sm. Needle & $1.20 \mu \mathrm{A}$ \\
\hline Sm. Needle w/ Humidifier & $0.48-0.66 \mu \mathrm{A}$ \\
\hline
\end{tabular}

Table II B

Modified Configuration vs. Corona Current

\begin{tabular}{|c|c|}
\hline Configuration & Current \\
\hline Bank Needle Insulated & Not detectable on $\mu \mathrm{A}$ scale \\
\hline $\begin{array}{c}\text { Bank Needle Insulated } \mathrm{w} / \\
\text { Humidifier }\end{array}$ & Not detectable on $\mu \mathrm{A}$ scale \\
\hline
\end{tabular}

What makes this more interesting is the effect that these factors may have on fiber formation during electrospinning. Obtaining well-formed fibers from this process has historically been a challenge. The results of this study may contribute to the understanding of the process parameters and apparatus configuration necessary to reproducibly generate well-formed fibers.

\section{References}

[1]

Ignatova, M. et. al. Study of Charge Storage in the Nanofibrous Poly(ethylene terephthalate) Electrets Prepared by Electrospinning or by Corona Discharge Method. www.elsevier.com European Polymer Journal Vol. 44, 2008.

Catalani, L. H., G. Collins, \& M. Jaffe. Evidence for Molecular Orientation and Residual Charge in the Electrospinning of Poly(butylenes terephthalate) Nanofibers. American Chemical Society @ 2007. van Turnhout, J. Thermally Stimulated Discharge of Electrets. Topics in
Applied Physics, vol. 33: Electrets, edited by G.M. Sessler. Darmstadt, Germany 1987.

Oda, T., K. Takashima, \& S. Ichiyama. Space and Surface Charge Behavior Analysis of Charge-Eliminated Polymer Films. IEEE Tokyo, Japan.

Kaneko, K., E. Nakane, \& T. Mizutani. Effects of Absorbed Water on Space Charge and Conduction Phenomena in Polyimide Films. IEEE 2005 Annual Report Conference on Electrical Insulation and Dielectric Phenomena.

Tripatanasuwan, S. \& D. H. Reneker. Corona Discharge from Electrospinning Jet of Poly(ethylene oxide) Solution. Elsevier $\odot 2009$.

Kumara, S. et. al. Charging of Polymeric Surfaces by Positive Impulse Corona. IEEE 2009.

Zhang, L., Z. Xu, \& G. Chen. Decay of Electric Charge on Corona Charged Polyethylene. Journal of Physics Vol. 142 Mianyang, China IOP Publishing Ltd. 2008.

Seanor, Donald A. Electrical Properties of Polymers. Academic Press, New York 1982.

Blythe, A.R. Electrical Properties of Polymers. Cambridge University Press 1979. 


\title{
Elevated Temperature Electrospinning of Aqueous Gelatin Solution and Crosslinking for Tissue Engineering Applications
}

\author{
Deep S. Pandya, Treena Arinzeh, George Collins \\ New Jersey Institute of Technology \\ University Heights, Newark, New Jersey 07102
}

\begin{abstract}
ECM is useful in providing growth and support to the cells as well as engineered tissues. There are various techniques for producing artificial matrix constructs to simulate the ECM. These include wet spinning, phase separation, dry spinning, and electrospinning. Electrospinning has advantages over other techniques as it can produce scaffolds which have a high surface area to volume ratio. Electrospinning is also capable of producing nanofibers from synthetic polymers as well as from natural polymers Ref. [1]. Gelatin has been electrospun from several different solvents Ref. [2]. In this work we have chosen water as the solvent because of its inherent noncytotoxicity. When gelatin is dissolved in water it will form a semi-solid gel. Heating the solution will prevent the formation of gel. Electrospun gel fibers will dissolve in water and physiological fluids. To overcome the gelation, an apparatus has been constructed that allows gel solution to be electrospun at elevated temperature.

As shown in Figure 1, the apparatus consists of a small insulated enclosure large enough to fit the electrospinning syringe. Heated air is supplied by a heating coil that is connected to a low pressure pump.
\end{abstract}

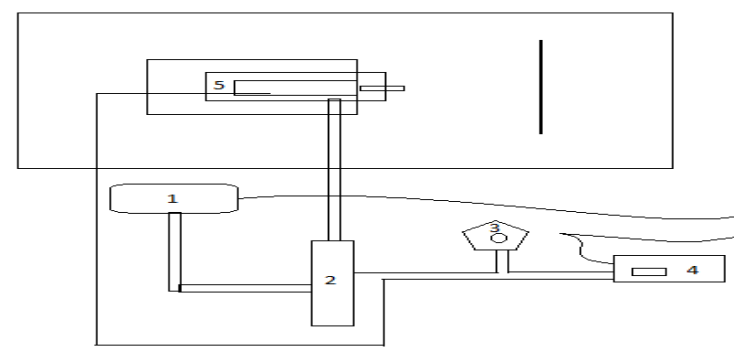

Fig.1. Schematic diagram of setup of elevated heating where 1 - Air pump, 2heat coil , 3 - Variac, 4- heat controller, 5- firm hood surrounding part of syringe
The heating coil is connected to the Variac by which air can be heated in proportion to the voltage. The Variac is connected to the temperature controller which can turn the Variac on and off relative to a set temperature.

The gelatin chosen for this study is Gelatin (225 bloom) type B from Bovine skin. Gelatin (24\%) should be stirred continuously in heated de-ionized water $\left(45^{\circ} \mathrm{C}\right)$ for two hours. A 12 gauge needle is used for spinning. The enclosure is heated for 10 minutes prior to electrospinning. A thermocouple has been place in the syringe to give temperature measurement to the temperature controller. A distance of 12-14 cm between collector plate and needle is set. Electrospinning is started with $24 \mathrm{kv}$ and initially flow rate of $1.2 \mathrm{ml} / \mathrm{hr}$ has been set. Various experiments showed that successful fiber production depended on the temperature of the solution and other parameters like flow rate, applied voltage supply and relative humidity, see Table 1 and 2 below. Temperature limit at any humidity was between $45-55^{\circ} \mathrm{C}$. Resulting fibers are smooth with diameters ranging from $450 \mathrm{~nm}$ to $3 \mu \mathrm{m}$. The initial morphological studies have suggested that fibers are not uniform in diameter.

TABLE 1

\begin{tabular}{|c|c|c|c|c|}
\hline \multicolumn{1}{|c|}{$\mathrm{RH}(40-59 \%)$} \\
$\begin{array}{c}\text { Concentrat } \\
\text { ion } \\
\mathbf{( \% )}\end{array}$ & $\begin{array}{c}\text { Distance } \\
\mathbf{( c m )}\end{array}$ & $\begin{array}{c}\text { Voltage } \\
\mathbf{( k v )}\end{array}$ & $\begin{array}{c}\text { Flow } \\
\text { rate } \\
\mathbf{( m l / h r )}\end{array}$ & $\begin{array}{c}\text { Resulting } \\
\text { diameter } \\
\text { (nm) }\end{array}$ \\
\hline 24 & $13-14$ & $23-24$ & $0.5-0$ & $450-3000$ \\
\hline 26 & $13-14$ & $23-24$ & 05.0 & $690-3000$ \\
\hline 30 & $14-16$ & $22-24$ & 1.2 & $2000-3000$ \\
\hline
\end{tabular}


TABLE 2

RH (14-30)

\begin{tabular}{|c|c|c|c|c|}
\hline $\begin{array}{c}\text { Concentrat } \\
\text { ion } \\
\mathbf{( \% )}\end{array}$ & $\begin{array}{c}\text { Distance } \\
\text { (cm) }\end{array}$ & $\begin{array}{c}\text { Voltage } \\
\text { (kv) }\end{array}$ & $\begin{array}{c}\text { Flow } \\
\text { rate } \\
\text { (ml/hr) }\end{array}$ & $\begin{array}{c}\text { Resulting } \\
\text { diameter } \\
\text { (nm) }\end{array}$ \\
\hline 24 & $14-18$ & $20-23$ & 1.2 & $450-5000$ \\
\hline 26 & $15-18$ & $20-23$ & 1.2 & $690-5000$ \\
\hline 30 & $14-20$ & $20-24$ & 1.2 & $2000-5000$ \\
\hline
\end{tabular}

Various crosslinking methods for the gelatin fibers were investigated. These include UV and dehydrothermal. Dehydrothermal crosslinking was performed in vacuum oven at $110^{\circ} \mathrm{C}$ for 48 and 72 hours. Crosslinked scaffold was immersed in de-ionized water to study the solubility. Fibers did not dissolve after 72 hours of treatment but lost the color and some shape in water. UV crosslinking was performed in laminar air flow hood for 30 minutes. Resulting fibers UV treated fibers did not dissolve in de-ionized water and relative to the dehydrothermal treated mats, held original shape. Fiber mats are removed from water and dried in vacuum oven for their further observation under scanning electron microscope to determine if the fibrous structure is maintained after being placed in water.

\section{$24 \%$ Gelatin}
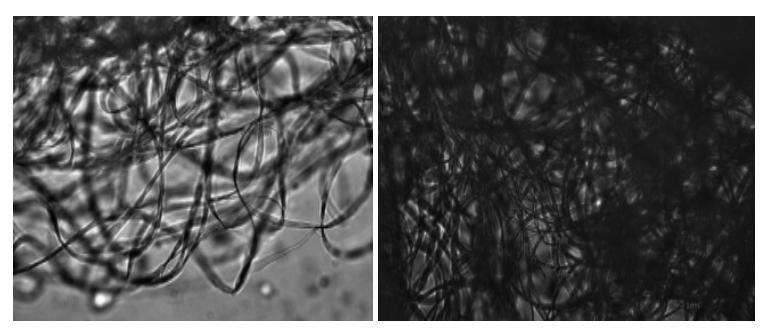

$26 \%$ Gelatin
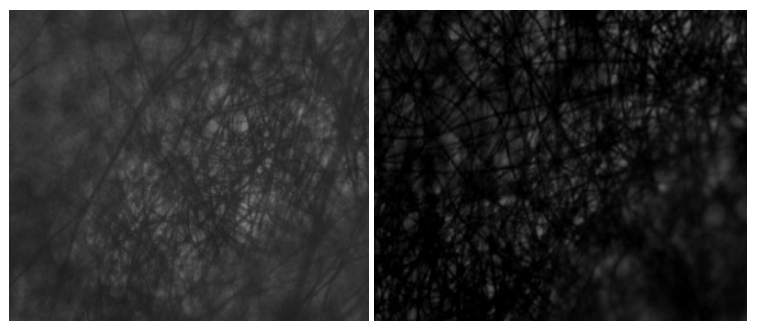

Fig.2. Four images of $24 \%$ gelatin top t taken at $50 \mathrm{X}$ zoom under optical microscope

\section{FUTURE WORK}

Future plan is to add crosslinkers directly to the gelatin solution, so that the electrospun fibers contain the crosslinker. This can be accomplished by adding natural crosslinkers such as genipin or enzyme transglutaminase just before the solution exits the needle from syringe. The idea will prevent the reaction to take place in gelatin/water solution. This will allow crosslinker to spin with gelatin and when gelatin is transformed form solution to solid fibers, the crosslinker will crosslink it. This approach has not been previously reported and can eliminate post electrospinning crosslinking steps.

\section{ACKNOWLEDGEMENT}

I want to thank Dr. Collins for believing in me and guiding me. I also want to thank Dr. Arinzeh and Dr. Zohar for spending their valuable time to guide my research. I would like to appreciate Yuki for sharing his experience of

\section{REFERANCES}

[1] L. Yang, C.F.C. Fitie, K.O. van der Werf, M.L. Bennink, P.J. Dijkstra and J. Feijen, Mechanical properties of single electrospun collagen type I fibers, Biomaterials 29 (2008), pp. 955-962. Article Cited By in Scopus

[2] Electrospun gelatin fibers : Effect of solvent system on morphology and fiber diameters ,CHOKTAWEESAP Nuanchan ; ARAYANARAKUL Kunawan ; AHT-ONG Duangdao; MEECHAISUE Chidchanok ; SUPAPHOL Pitt

[3] Electro-spinning of pure collagen nano-fibres - Just an expensive way to make gelatin? Dimitrios I. Zeugolisa, b, c, 1, Shih T. Khewd, Elijah S.Y. Yewb, Andrew K. Ekaputrab, Yen W. Tongb, d, Lin-Yue L. Yungd, Dietmar W. Hutmacherb, e, 2, Colin Sheppardb and Michael Raghunath 


\title{
Effect of Hydroxyapatite Particles on Stem Cell Response in Nanofiber Scaffolds
}

\author{
${ }^{1}$ S.P. Kwei, ${ }^{1}$ K.L. Moffat, ${ }^{2}$ S. Doty, and ${ }^{1}$ H. H. Lu \\ ${ }^{1}$ Department of Biomedical Engineering, Columbia University, ${ }^{2}$ Orthopaedic Surgery, Hospital for Special Surgery, NY, NY
}

\begin{abstract}
Rotator cuff tears are among the most common shoulder injuries that require surgery. High failure rates of biological graft-based repairs underscore the need for functional alternatives. Specifically, functional grafts must incorporate the gradient of mineralization from tendon to bone in order to be biomimetic. In this study, effects of varying concentrations of mineral content in aligned nanofiber scaffolds on human mesenchymal stem cells (hMSC) are evaluated. It is hypothesized that mineral content will regulate cell response, matrix deposition, and integrin gene expression. hMSC were seeded on aligned nanofiber scaffolds of polylactide-co-glycolide with $0 \%$, $10 \%$, and $15 \%$ hydroxyapatite content, and were maintained in chondrogenic medium. Cell proliferation $(n=5)$, collagen deposition $(n=5)$, and gene expression $(n=5)$ for Collagen $X$, Sox9, osteopontin, osteonectin, and osteocalcin were determined over 42 days. Cell number was found to differ between the nonmineralized and mineralized groups. Significant increase in collagen deposition over time was observed in mineralized scaffold groups, and the $15 \%$ group showing significantly higher deposition than the $0 \%$ group by day 42 . Lower expressions of chondrocyte hyperotrophy marker Collagen $X$ and chondrogenic marker Sox9 and maintained high expressions of osteogenic markers osteopontin, osteonectin, and osteocalcin suggest an osteogenic lineage for the stem cells. In conclusion, addition of HA particles influenced hMSC proliferation, matrix deposition, and may induce an osteogenic differentiation response from the stem cells.
\end{abstract}

\section{INTRODUCTION}

Rotator cuff tendon repair is one of the most common shoulder procedures performed annually in the United States[1]. Full-thickness rotator cuff tears often result from avulsion of the tendon from the bone at the insertion site, which is not regenerated following repair. To improve tendon repair, we have developed a degradable polymer nanofiberbased scaffold system[2], and are currently evaluating the response of human mesenchymal stem cells (hMSC) on this novel scaffold. The tendon-to-bone insertion site is a complex and heterogeneous structure, with a series of interface transitions from non-mineralized fibrocartilage to mineralized fibrocartilage[3]. Our working hypothesis is that the regeneration of the tendon-to-bone insertion is critical for integrative rotator cuff repair to ensure long-term stability and greater functionality. The objective of this study is to evaluate hMSC responses to varying concentrations of hydroxyapatite (HA) in nanofiber scaffolds maintained in chondrogenic medium. It is hypothesized HA presence will influence matrix deposition and gene expression, and provide insight to the differentiation pathway of the stem cells.

\section{MATERIALS AND METHODS}

\section{Scaffold Fabrication}

Aligned nanofiber scaffolds of poly(lactic-co-glycolic) acid (PLGA 85:15, Lakeshore) with $0 \%, 10 \%$, and 15\% HA (wt/vol) were produced by electrospinning[2].

\section{Cells and Cell Culture}

Human mesenchymal stem cells (hMSC, Cambrex, passage $=9$ ) were seeded on the nanofiber scaffolds at a density of $3.14 \times 10^{4}$ cells $/ \mathrm{cm}^{2}$ and maintained in chondrogenic fully supplemented medium containing 10\% ITS, 10\% fetal bovine serum (FBS), $40 \mu \mathrm{g} / \mathrm{mL}$ L-proline, $100 \mu \mathrm{g} / \mathrm{mL}$ sodium pyruvate, $0.1 \mu \mathrm{M}$ dexamethasone, $50 \mu \mathrm{g} / \mathrm{mL}$ ascorbic acid, $1 \%$ antibiotics, $1 \%$ non-essential amino acids, and $0.1 \%$ antifungal.

\section{End-point Analyses (1,3, 7, 14, 28, 42 days)}

Cell proliferation $(n=5)$ was evaluated on days $1,7,14,28$, and 42 by measuring total DNA content using PicoGreen dsDNA assay (Invitrogen) following manufacturer's suggested protocol. Total collagen $(\mathrm{n}=5)$ was measured on days $1,7,14$, 28 , and 42 by the SIRCOL kit according to the provided protocol. Gene expressions $(n=3)$ were measured on days 3 , 14, 28, and 42 for Collagen X, Sox9, osteopontin, osteonectin, and osteocalcin were evaluated by RT-PCR.

\section{RESULTS}

Cell proliferation was continuously observed for $0 \% \mathrm{HA}$ group, while the $10 \%$ and $15 \%$ HA groups showed distinct cell proliferation behavior (Fig. 1A). Cell number for 10\% HA group decreased significantly after day 28 , while cell number for the $15 \%$ scaffold group decreased after day 14 and increased on day 42 .

Total collagen deposition (Fig. 1B) was observed to be higher on mineralized scaffolds. Specifically, the $15 \%$ HA group showed significant increase in and significantly higher collagen deposition by day 42 . In addition, the $10 \%$ HA group showed significant increase in collagen deposition from days 14 to 42 . In contrast, the $0 \%$ HA group showed no significant differences over time.
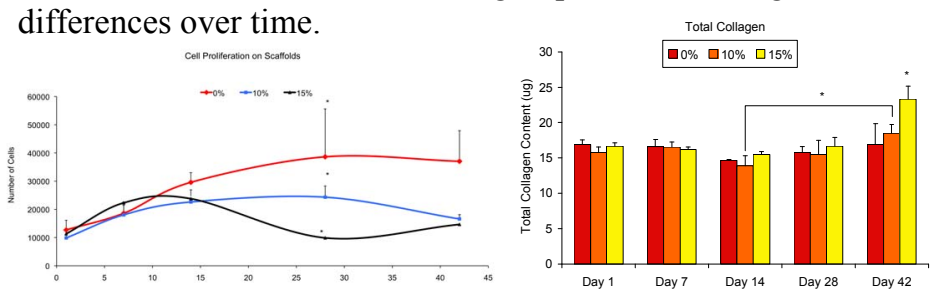

Figure 1: A) Cell proliferation and B) total collagen content for $0 \%, 10 \%$, and $15 \%$ PLGA-HA scaffold groups, ${ }^{*}: \mathrm{p}<0.05$. 
Distinct gene expression profiles were observed on nonmineralized and mineralized scaffolds for chondrogenic markers Collagen X and Sox 9 (Fig. 2). Gene expression for $10 \%$ and $15 \%$ HA groups were significantly lower than the $0 \%$ HA group in the later time points.

A
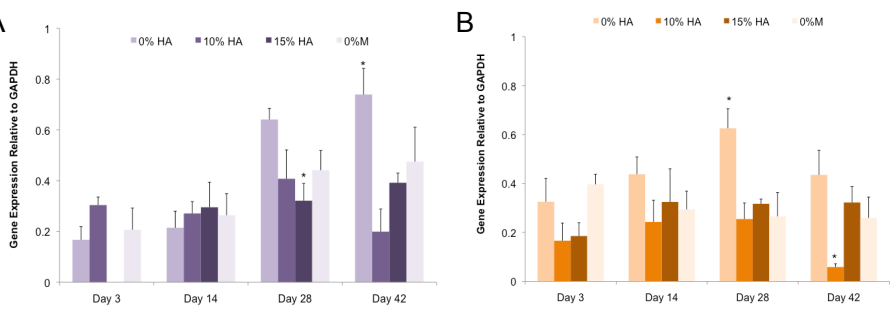

Figure 2: Normalized gene expression for $0 \%, 10 \%$, and 15\% PLGA-HA scaffold groups for A) Collagen X and B) Sox9, *: $\mathrm{p}<0.05$.

In contrast, gene expressions for osteogenic markers osteopontin, osteonectin, and osteocalcin remained consistently high over time for all scaffold groups (Fig. 3).
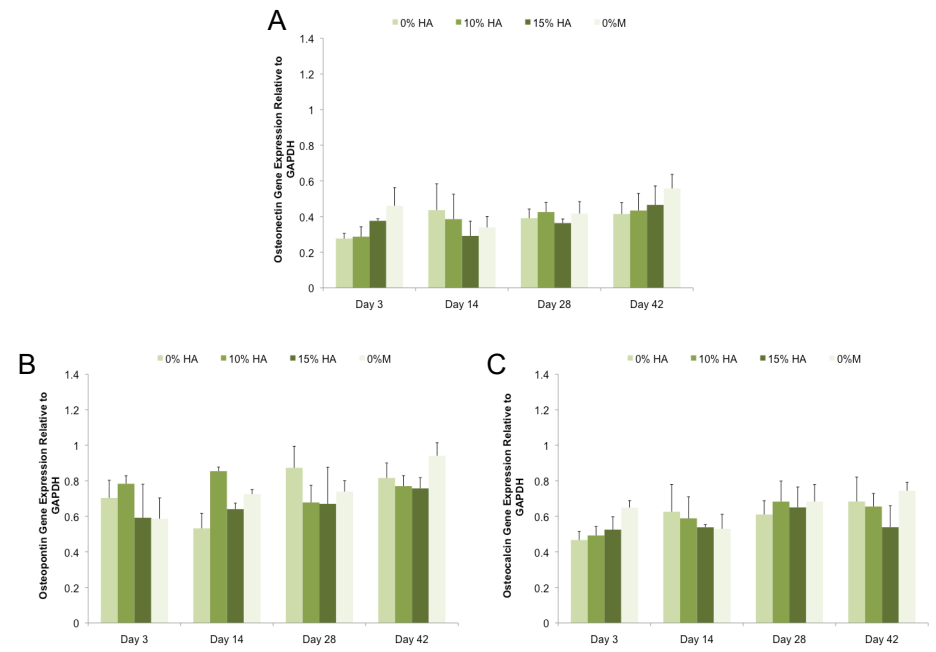

Figure 3: Normalized gene expression for $0 \%, 10 \%$, and $15 \%$ PLGA-HA scaffold groups for A) osteonectin, B) osteopontin, and C) osteocalcin, $*$ : $\mathrm{p}<0.05$.

\section{DISCUSSION}

The presence of HA particles in the scaffolds has produced distinct responses from the cells in proliferation, matrix deposition, and gene expression. For cell proliferation, the $0 \%$ HA scaffold group showed continuous growth, while the $10 \%$ HA and $15 \%$ HA scaffold group showed fluctuating growth at later time points. Reasons for disturbance in cell growth on mineralized scaffolds may environmental. Specifically the nanofiber scaffold becomes more textured with the addition of the particles, which may affect cell attachment and adhesion. Alternatively, from a differentiation perspective, the lag in cell proliferation for mineralized scaffold groups may be attributed to the impending differentiation of the stem cells, pausing cell growth for this process.
Collagen deposition is important for both tendon and bone construction. Mineralized scaffold groups showed significantly higher collagen deposition in the later time points than the non-mineralized scaffold group. The presence of the HA particles may induce a response from the stem cells to deposit collagen.

Gene expression data can be helpful in predicting cell response and activity. The stem cells were cultured in chondrogenic medium, which could direct the stem cells on a chondrogenic differentiation pathway. However, the HA particles may have counteracted the media's effect, as expressions on $10 \%$ and $15 \%$ HA groups for both chondrogenic markers Collagen $\mathrm{X}$ and Sox 9 are significantly lower than that of the 0\% HA group. Furthermore, all groups retained high expressions for osteogenic markers osteopontin, osteonectin, and osteocalcin over time. The downregulation of chondrogenic markers and maintained, high expression for osteogenic markers suggest that the presence of HA particles may direct the stem cells toward a more osteogenic differentiation pathway. This may be expected as HA is found in bone, and its presence may indicate to the stem cells a more bone-like environment.

Limitations in the study include the high passage of the stem cells. Stem cells at such a high passage may lose their native qualities, affecting cell response. It would be pertinent to repeat the study on a smaller scale with stem cells at a lower passage and reevaluate cell response. Furthermore, while the cells were cultured in chondrogenic medium, the effects of the medial seemed to be overpowered by the presence of the HA particles. In order to induce a more chondrogenic differentiation response from the stem cells, an environment with higher concentrations of chondrogenic agents would be considered for future studies.

\section{ACKNOWLEDGEMENT}

The study was supported by the National Institute of Health, National Institute of Arthritis and Musculoskeletal and Skin Diseases (NIH/NIAMS R21-AR056459, R01AR055280).

\section{REFERENCES}

[1] Vitale, M. A., Vitale, M. G., Zivin, J. G., Braman, J. P., Bigliani, L.U. \& Flatow, E. L. Rotator cuff repair: an analysis of utility scores and cost-effectiveness. J Shoulder. Elbow. Surg 16, 1812007.

tears. J. Bone Joint Surg. Am. 86-A, 2192004.

[2] Moffat, K. L., Kwei, S. P., Spalazzi J. P., Doty, S., Levine, W., Lu, H. H. Novel Nanofiber-Based Scaffold for Rotator Cuff Repair and Augmentation. Tissue Eng 15. 115 2009.

[3] Thomopoulos S, Williams, G. R., Gimbel J. A., Favata M, Soslowsky L. J. Variation of biomechanical, structural, and compositional properties along the tendon to bone insertion site. J. Orthopaedic Research 21(3), 413, 2003. 


\title{
Optimization of the Electrospinning Process Parameters for a Pandemic Vaccine Patch
}

\author{
Derya Karatas, K.M. Sawicka, S.R. Simon \\ Stony Brook University \\ Basic Science Tower 9-148 \\ Stony Brook, NY 11794-8691
}

\begin{abstract}
A skin patch composed of an electrospun nanofibrous membrane of a highly hygroscopic polymer, encasing a reversibly packaged antigen and adjuvant cocktail, promises a practical alternative to the current vaccine strategy. The proposed system would utilize the high density of antigen presenting (APC) cells found within the layers of human skin to elicit a vast adaptive immune response. The amount of contact between patches and skin has been said to directly affect the efficiency of load delivery. Incorporation of the immunogens often requires use of aqueous solvents associated with the beads-on-the-string morphology formation, which hinders the high surface area to volume ratio afforded by the electrospun membranes. The long-term goal of this research is to optimize the solution and process parameters to maximize the surface interaction with the skin for improved delivery of immunogens. In this study we attempt to utilize a novel method of morphology control through employment of the electrospinning process pausation. The scanning electron microscopy (SEM) examination indicated that stopping the electrospinning process at different intervals and for various durations impacts the generated morphology. The findings indicate that the surface area to volume ratio for the threedimensional nonwoven membrane can be maximized through utility of process pausing.
\end{abstract}

\section{INTRODUCTION}

The current vaccination method of the intramuscular injection falls short during pandemic situations, which call for rapid and efficient mass immunization. The vaccine patch proposed by our lab promises a practical alternative by utilizing the electrospun membranes to deliver immunogens into the body's primary defense organ. The protein stability demands the use of aqueous solvents which result in bead formation and therefore hinder the unmatched surface area to volume ratio offered by the nanofibrous membrane. The main goal of this study was to explore the effects of various system pausing rates on the morphology of these fibers such that there will be an improvement on this surface area to volume ratio.

\section{METHODS AND MATERIALS \\ A. Solution Preparation}

Two concentrations of polyvinyl pyrrolidone (PVP), (Sigma Aldrich, St. Louis, MO) were electrospun. Both the $0.10 \mathrm{mM}$ and the $0.05 \mathrm{mM}$ PVP solutions were dissolved in a mixture of $75 \%$ by volume absolute ethanol and $25 \%$ by volume $0.1 \%$ bovine serum albumin (BSA) (Sigma Aldrich, St. Louis, MO) in phosphate buffered saline (PBS). B. Electrospinning

The electrospinning process uses an electric field to break the surface tension of a liquid polymer droplet to transform it into a charged jet, which undergoes solvent evaporation as it travels towards a grounded collector, where it deposits as a nonwoven mat of solid nanofibers. Throughout the study the voltage of $15.0 \mathrm{kV}$ and distance between needle and collector of $10.0 \mathrm{~cm}$ was maintained. The solutions were electrospun at flow rates of $5 \mu \mathrm{L} / \mathrm{min}, 10 \mu \mathrm{L} / \mathrm{min}$ or $20 \mu \mathrm{l} / \mathrm{min}$ with the total volume spun was $250 \mu \mathrm{L}$. The process was stopped every $100 \mu \mathrm{L}$ for 30,45 or 60 seconds. The experiments were carried out at ambient humidity and temperature.

C. SEM imaging

A representative area of each sample was taped onto an aluminum plate using aluminum tape and images of samples were obtained using a scanning electron microscope.

\section{RESULTS}

The electrospinning process has been proposed for development of a novel vaccine patch that would surpass the predicaments of the current vaccine paradigm. 


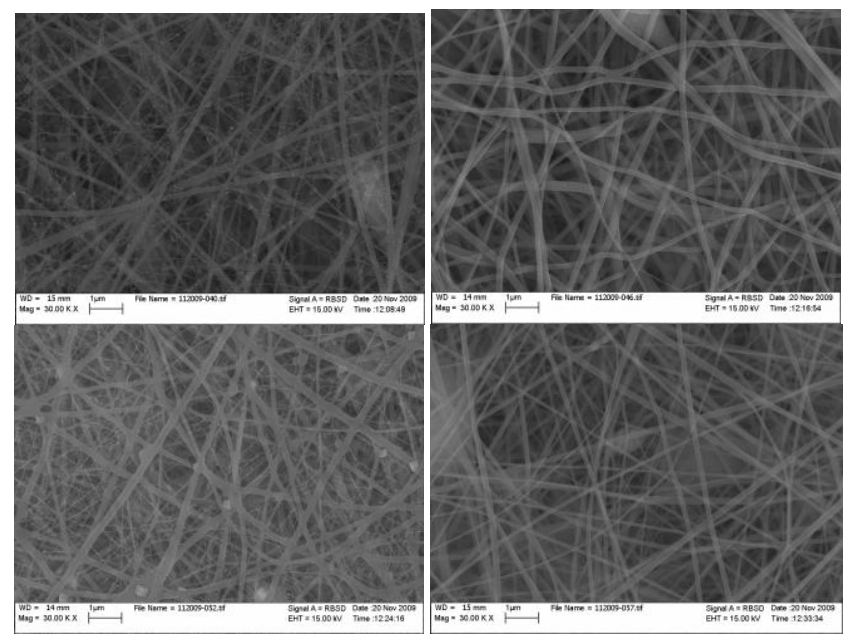

Figure 1 (from top left to bottom right) $0.10 \mathrm{mM} \mathrm{PVP}$

5 $\mu \mathrm{L} / \mathrm{min} \mathrm{Ctrl}, 30,45$, and 60sec pauses

The inclusion of immunogens into the electrospinning solution results in a bead-on-thestring morphology formation caused by the addition of aqueous solvents required to stabilize the protein. The beading hinders the large surface area to volume ratio typically obtained by the electrospinning method. This study aimed to control the membrane morphology through employment of the electrospinning process pausation. The various system pausing rates were found to affect the fiber and bead size. There appeared to be a threshold within pausing duration between 30 and 45 seconds. Comparison of the two concentrations showed that increasing the concentration from $0.05 \mathrm{mM}$ to $0.1 \mathrm{mM}$ created fibers with greater uniformity and decreased the beading frequency.

\section{CONCLUSION}

The electrospun nanofibrous membrane proposed for pandemic vaccine alternative aims to deliver immunogens into the APC rich region of the human skin. To achieve delivery the membrane morphology has been be tailored to provide a maximized surface area to volume ratio. By controlling the amount of beading that occurs as a result of the aqueous solvent required for protein stability. The findings of this study indicate that the morphology of the three-dimensional nonwoven membrane can be maximized through utility of the electrospinning process pausing. In the future the study will be expanded to incorporate horseradish peroxidase (HRP) to quantify the effect of pausing on efficacy of protein encapsulation.

\section{REFERENCES}

[1] K.M. Sawicka, P. Gouma, "Electrospun composite nanofibers for functional applications," J. Nanoparticle Research, vol.8, pp. 769- 781, 2006 [4] G.M. Glenn, D.N. Taylor, X. Li, S. Frankel, A. Montemarano, and C.R. Alving, "Transcutaneous immunization: A human vaccine delivery strategy using a patch," Nature Medicine, vol. 6(12), pp. 1403-6, 2000. 


\title{
In Vitro Characterization of Polymer-Ceramic Nanofiber Scaffolds
}

\author{
S.E. Gordon, K.L. Moffat, and H. H. Lu \\ Department of Biomedical Engineering, Columbia University \\ 500 West $120^{\text {th }}$ Street, New York, NY 10027
}

\begin{abstract}
Rotator cuff tears often occur at the tendon-bone insertion site, a fibrocartilaginous interface that is not regenerated during current repairs. Our aim is to regenerate the insertion site through use of extracellular matrix based scaffolds. In this study, we evaluated the response of mineralized and nonmineralized nanofiber scaffolds to cell culture environment without the influence of cell growth and matrix deposition. We observed minimal change in scaffold mineral content, composition, and chemistry over culture time. Structural properties were influenced, in particular, fiber diameter increased throughout culture. This data is essential for scaffold characterization and understanding cell-material interactions.
\end{abstract}

\section{INTRODUCTION}

Each year, approximately 300,000 rotator cuff repairs are performed in the United States. However, the failure rate of primary cuff repairs is reported to be as high as $94 \%$ [1-2]. Most rotator cuff tears occur at the tendon-bone insertion site; therefore tendon to bone repair is required. The native tendon-to-bone insertion site is a direct insertion site which is composed of fibrocartilage, which is comprised of both nonmineralized and mineralized regions. The tendon-to-bone insertion site is essential for proper load transfer between tendon and bone and musculoskeletal function. This critical interface is not regenerated during tendon-to-bone healing, rather a fibrovasuclar tissue with weak mechanical properties is formed [3]. The lack of insertion site regeneration destabilizes the repair site and does not allow for proper load transmission from muscle to bone, leading to rotator cuff repair failure [4-6]. The high failure rates of rotator cuff tendon repair procedures coupled with the lack of insertion site regeneration indicate the significant clinical need for functional solutions to improve rotator cuff repairs.

To address this clinical need, tissue engineering-based strategies are being investigated for augmenting current rotator cuff repair procedures. Previous studies have focused on the use of nanofiber scaffolds to regenerate both the tendon and fibrocartilage regions of the tendon-to-bone insertion site[7]. In these studies it was determined that nanofiber scaffolds composed of aligned fibers of polylactide-coglycolide (PLGA, 85:15) mimic the structural and mechanical properties of rotator cuff tendons and that fiber organization guides the alignment of human rotator cuff tendon fibroblasts and the resulting matrix deposition. Additionally, PLGA nanofiber scaffolds were evaluated for non-mineralized and mineralized fibrocartilage formation [7]. To mimic the mineral phase of the mineralized fibrocartilage region, nanoparticles of hydroxapatite (HA) were incorporated into the nanofibers. It was determined that both PLGA and PLGA-HA mimic the mechanical properties of insertion site non-mineralized and mineralized fibrocartilage, respectively, and support the formation of a fibrocartilage-like matrix in vitro [7].

Building on these promising results, the goal of this study is to characterize the PLGA and PLGA-HA scaffolds as a function of culture, without the presence of cells. Specifically, the objectives are two-fold: 1) to evaluate the scaffold structural properties and 2) to examine the mineral content, composition, and chemistry as a function of in vitro culture. It is hypothesized that scaffold degradation and mineral dissolution will occur over time. Understanding the influence of culture conditions on scaffold properties without the influence of cell growth and matrix deposition is essential for scaffold characterization and evaluating the effect of scaffold properties on cell behavior.

\section{MATERIALS AND METHODS}

Scaffold Fabrication: Aligned nanofiber scaffolds composed of PLGA (85:15, Lakeshore Biomaterials) and PLGA-HA (nano-HA, Nanocerox, 100-150nm) were produced via electrospinning, as previously described [8]. Specifically, scaffolds containing $0 \%, 10 \%$, and $15 \%$ HA were formed. A rotating collecting target was utilized to form aligned fibers. In Vitro Culture Conditions: Scaffolds were immersed in human plasma fibronectin $(10 \mu \mathrm{g} / \mathrm{mL})$ for 12 hours to mimic cell culture conditions,. Scaffolds were cultured over 42 days at $37^{\circ} \mathrm{C}$ and $5 \% \mathrm{CO}_{2}$. Media, composed of DMEM, $10 \%$ FBS, $1 \%$ ITS + premix, $1 \%$ NEAA, $1 \%$ antibiotics, $0.1 \%$ antifungal, $0.05 \%$ sodium pyruvate, $0.05 \%$ L-proline, $50 \mu \mathrm{g} / \mathrm{mL}$ ascorbic acid, and 100nmdexamethasone, was collected and changed twice per week. End-Point Analyses (at 0, 1, 7, 14, 28, and 42 days: Structural Properties: Nanofiber morphology was assessed by Scanning Electron Microscopy (SEM, FEI Quanta 600, n=3) and image analysis was used to determine fiber diameter (ImageJ, $\mathrm{n}=18$ ). $\underline{\text { Scaffold Mineral }}$ Content, Composition, and Chemistry: Scaffold mineral content was evaluated by Thermogravimetric analysis (TGA, $\mathrm{n}=4$ ) and qualitative and quantitative Alizarin Red assays (ALZ, n=5). Mineral composition was determined with Energy Dispersive X-ray Analysis (EDAX,n=3). Scafflold mineral chemistry was evaluated by Fourier Transform 
Infrared Spectroscopy (FTIR, n=3). Statistical Analysis: Results are reported as average \pm standard deviation with $\mathrm{p}<0.05$, and two way ANOVA was performed followed by Tukey HSD post-hoc test for all pair-wise comparisons.

\section{RESULTS}

Structural Properties: Fiber morphology was found to uniform for each group (Fig. 1). The nanofibers were cylindrical and aligned for all groups. For the groups containing mineral appeared to have a rougher surface morphology than the PLGA nanofibers which appear smooth. Fiber dimensions (Table 1) changed over time, with larger diameters at later time points. There was a significant increase in diameter for all groups by day 42. Larger fiber diameters were found for PLGA scaffolds compared to PLGA-HA at all time-points, with the exception of $0 \%$ and $10 \%$ HA on day 14 . Scaffold wet weight was constant over culture time.

Table 1: Scaffold Characterization

\begin{tabular}{|c|c|c|c|}
\hline \hline Day & $0 \% \mathrm{HA}$ & $10 \% \mathrm{HA}$ & $15 \% \mathrm{HA}$ \\
\hline \multicolumn{4}{|c|}{ Fiber Diameter } \\
\hline 0 & $0.7 \pm 0.3 \mu \mathrm{m}$ & $0.5 \pm 0.1 \mu \mathrm{m}$ & $0.3 \pm 0.1 \mu \mathrm{m}$ \\
\hline 14 & $0.6 \pm 0.1 \mu \mathrm{m}$ & $0.6 \pm 0.2 \mu \mathrm{m}$ & $0.5 \pm 0.1 \mu \mathrm{m}$ \\
\hline 42 & $0.9 \pm 0.2 \mu \mathrm{m}$ & $0.8 \pm 0.2 \mu \mathrm{m}$ & $0.6 \pm 0.1 \mu \mathrm{m}$ \\
\hline \multicolumn{4}{|c|}{ Thermogravimetric Analysis (TGA, weight percent) } \\
\hline As-Fabricated & $3.0 \pm 0.3$ & $12.7 \pm 0.8$ & $17.9 \pm 0.6$ \\
\hline 0 & $3.8 \pm 0.7$ & $13.0 \pm 1.9$ & $33.2 \pm 11.1$ \\
\hline 14 & $4.7 \pm 4.0$ & $16.2 \pm 1.8$ & $18.7 \pm 13.2$ \\
\hline 42 & $2.3 \pm 0.4$ & $18.8 \pm 4.8$ & $30.2 \pm 14.6$ \\
\hline
\end{tabular}

Scaffold Mineral Content, Composition and Chemistry:

Thermo-gravimetric analysis confirmed the percent mineral incorporated into the PLGA-HA scaffolds during fabrication (Table 1). Additionally, TGA indicated that scaffold mineral content did not change significantly over time. EDAX confirmed that PLGA-HA scaffolds contain calcium and phosphorous, the mineral components of hydroxyapatite (Fig. 1). Additionally, the $\mathrm{Ca} / \mathrm{P}$ ratio was similar for mineral containing scaffolds and remained constant throughout culture time. Analysis of mineral chemistry with FTIR revealed that peaks for both PLGA $\left(\mathrm{C}=\mathrm{O}, 1780 \mathrm{~cm}^{-1}\right)$ and $\mathrm{HA}\left(\mathrm{PO}_{4}, 570\right.$ $\mathrm{cm}^{-1}, 600 \mathrm{~cm}^{-1}$ ) were observed at all time points and similar peaks were found for all mineral containing scaffolds. Variations in nanofiber roughness were observed at all timepoints, which was due to the presence of nano-HA. ALZ showed fluctuation over time, but the changes were not significant.

\section{DISCUSSION AND CONCLUSIONS}

The goal of this study was to evaluate the effect of in vitro

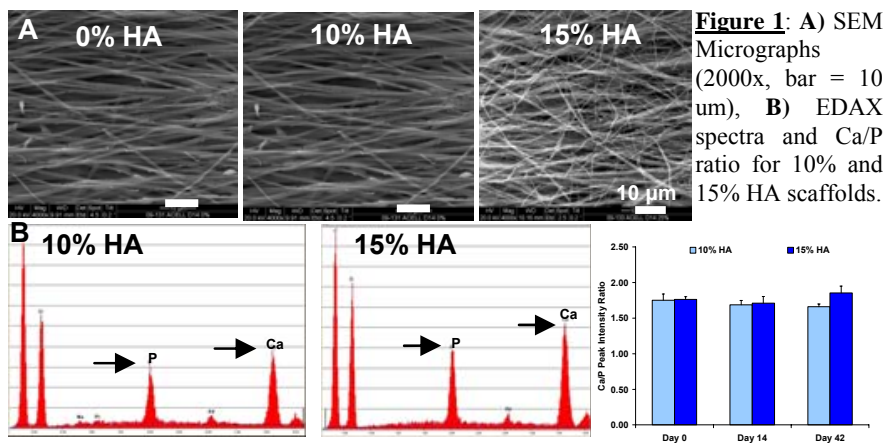

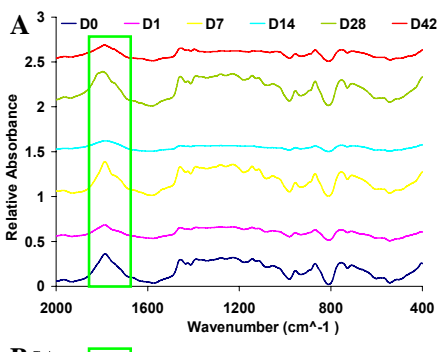

Figure 2: FTIR spectra from 2000 $400 \mathrm{~cm}^{-1}$ for A) $0 \%$ B) $10 \%$, and C) $15 \%$ HA. The green rectangle highlights the $\mathrm{C}=\mathrm{O}$ peak, characteristic of PLGA. The red rectangle highlights phosphate bending peaks, characteristic of HA.
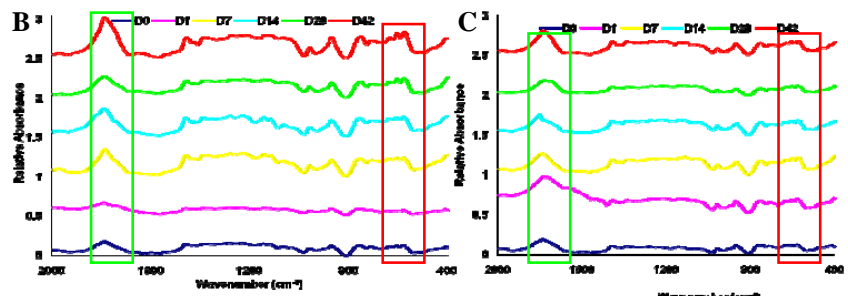

culture conditions on the structural properties and mineral behavior of PLGA and PLGA-HA nanofiber scafffolds. An increase in fiber diameter was observed over time for all

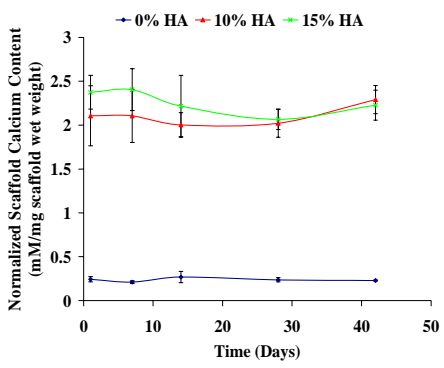

groups, which suggests water absorption over time. Minimal change in mineral content, composition and chemistry of PLGAHA nanofiber scaffolds was observed throughout culture. Interestingly, the $\mathrm{Ca} / \mathrm{P}$ ratio of the PLGA-HA nanofiber scaffolds was found to be

Figure 3: Scaffold Calcium Content (ALZ assay) similar to that of native mineralized tissue (1.67) which is

beneficial for matrix mineralization and osteointegration. Taken collectively, these data suggest that in vitro culture conditions influence the scaffold fiber dimensions but do not strongly alter the scaffold mineral properties. Future work will focus on utilizing this data to understand cell-material interactions.

\section{ACKNOWLEDGEMENT}

The study was supported by the National Institute of Health, National Institute of Arthritis and Musculoskeletal Skin Diseases (NIH/NIAMS R21-AR056459, R01-AR055280).

\section{REFERENCES}

[1] A. Aurora, et al., J Shoulder Elbow Surg., 2007.

[2] L. Galatz, J. Bone Joint Surg. Am., 2004.

[3] S. Rodeo, Clin. Sports Med.,2009.

[4] M. Benjamin, J. Anat,., 1986.

[5] R. Cooper, S. Misol, J. Bone Joint Surg Am., 1970.

[6] S. Woo et al., American Academy of Orthopaedic Surgeons, 1988.

[7] K. Moffat, Proceedings, Society for Biomaterials, 2009.

[8] K. Moffat et al, Tissue Eng. 2009. 


\title{
Multifunctional Superparamagnetic Iron-Oxide Nanoparticles (SPION) for Treating Orthopedic and Biofilm Infections
}

\author{
Erik N. Taylor and Thomas J. Webster \\ Division of Engineering and Orthopedics \\ Brown University \\ Providence, RI 02917 USA
}

\begin{abstract}
As with all surgical procedures, implantation comes with the added risk of infection. The inability of antibacterial therapy to treat persistent infection demonstrates the need for a more advanced therapeutic approach. The goal of this proposal is, for the first time, to use superparamagnetic iron oxide nanoparticles (SPION) to treat infection on biomedical devices. The hypothesis of this study is that SPION could be used as a modular tool for comprehensive infection treatment including disease targeting, infection control, and therapy feedback.
\end{abstract}

\section{INTRODUCTION}

Infection has been reported on an array of implantable devices including joint prostheses, central venous catheters, endotracheal tubes, mechanical heart valves, and others [1]. Biofilm bacteria are resistant to antibiotic treatment and therefore infections are recalcitrant without prolonged treatment (which promotes emergence of antibiotic resistant strains) or removal of the device (which can debilitate the patient) [2]. Moreover, it is clear that antibiotic resistant organisms (such as methicillin resistant $S$. epidermidis and MRSA) are spreading in the hospital setting. A recent report estimates that the number of MRSA infections in hospitals has doubled nationwide, from approximately 127,000 in 1999 to 278,000 in 2005 [3]. The difficulty antibiotics have in treating resistant infection demonstrates the need for a different novel approach. The goal of this study was to explore the use of superparamagnetic iron oxide nanoparticles (termed SPION here) as a multifunctional platform for the inhibition of bacteria biofilm formation enabled by size-dependent material properties, surface modification, and coatings. It is believed that SPION could be used for various infection related applications.

In this study, SPION were coated with Dimercaptosuccinic acid (DMSA) providing a multifunctional linker which can be used to conjugate various useful chemistries. Here, nanoparticles were coated with silver and zinc to improve antibacterial activity. DMSA can also be used to functionalize SPION via sulphur-maleimide interactions with antibodies such as antiprotein A which would target SPION to a Staphylococcus aureus in biofilms. Once at the target site, SPION could provide antibiotic therapy, therapeutic feedback (via MRI), and even delivery of other drugs (Figure 1).

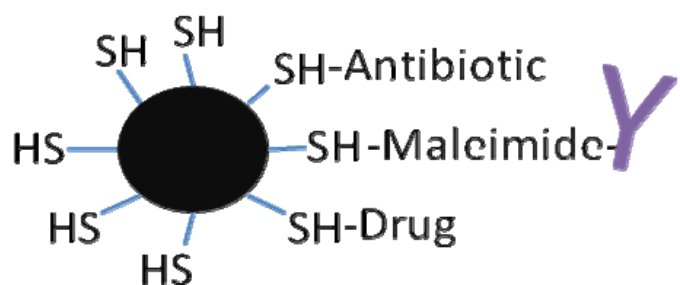

Figure 1. Schematic of multifunctional SPION. Free thiol groups of dimercaptosuccinic acid (DMSA) can be used to conjugate various useful chemistries such as antibiotics, antibodies, and drug molecules.

\section{METHODS}

\section{A. DMSA-SPION Synthesis}

Particle synthesis was carried out by dissolving 1 mmol $\mathrm{Fe}(\text { acac })_{3}$ in triethylene glycol. SPION formation occurred during reflux at $273^{\circ} \mathrm{C}$ for 30 minutes. After cooling to room temperature, SPION were coated with dimercaptosuccinic acid (DMSA-SPION) and precipitated with ammonium sulfate in the presence of a magnetic field. B. Antibacterial Conjugation to SPION

Silver nitrate, zinc chloride, iron chloride, or gallium nitrate were added to $1 \mathrm{mg} / \mathrm{ml}$ of a stock SPION solution in ethanol at either 1 or $4 \mathrm{mmol}$. Solutions were then vortex mixed and sonicated to aid in salt dissolution. Prepared nanoparticle mixtures were placed on a plate shaker overnight at $\sim 80$ RPM and then precipitated in the presence of a magnetic field. This was repeated three times to ensure there was no non-magnetic metal salt remaining in the solution.

\section{Nanoparticle Characterization}

This study determined the size and magnetic properties. Transmission electron microscopy (TEM) was used to determine particle size. Magnetic properties were verified qualitatively by placing a centrifuge tube containing SPION dissolved in ethanol on a plate shaker in the presence of a magnetic field.

D. Bacteria Assay

Bacteria utilized were frozen Staphylococcus aureus (ATCC \#25923) stock cultured in Tryptic Soy Broth (TSB) at $200 \mathrm{rpm}$ for 18-24 hours until late stationary phase was reached. Bacteria were diluted at a ratio of 1:100 in TSB or 
TSB containing coated SPION and seeded onto a 96-well plate for 24 hours (to allow formation of a biofilm). At this time, SPION with various metal coatings ( $\mathrm{Zn}, \mathrm{Ag}, \mathrm{Fe}, \mathrm{Ga}$ ) were added. After an additional 24 hours, the average biofilm density was analyzed using a $1 \%$ crystal violet solution in water. This solution was added to the biofilms at $125 \mu \mathrm{L}$, rinsed three times in PBS, dissolved in absolute ethanol, and analyzed by a spectrophotometer at $562 \mathrm{~nm}$.

\section{RESULTS}

\section{A. Nanoparticle Characterization Results}

Results of the present study demonstrated a simple method for the synthesis of water soluble SPION which

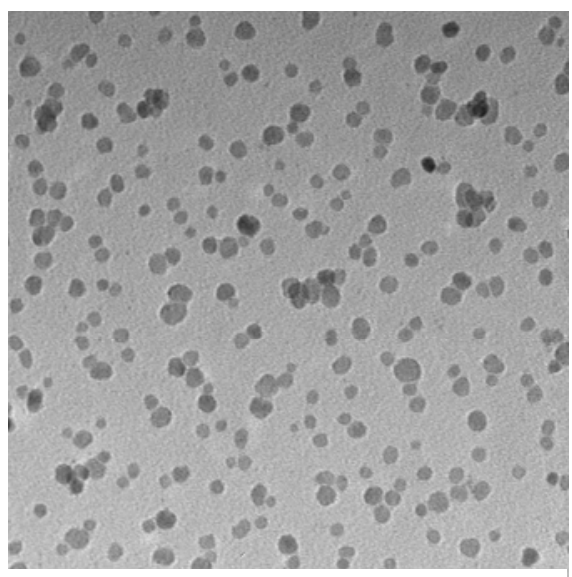

Figure 2. TEM analysis of SPION produced by refluxing $\mathrm{Fe}(\mathrm{acac})_{3}$ in triethylene glycol. Average particle size is estimated to be $10 \mathrm{~nm}$.

\section{A. Bacteria Assay Results}

Moreover, bacteria results in the presence of antibacterial conjugated SPION for up to 48 hours demonstrated anti-biofilm activity for all coated samples except gallium. Additionally, uncoated SPION (DMSA capped without any metal) indicated for the first time decreased bacteria density compared to no particles (0 $\mathrm{mg} / \mathrm{ml}$ or control) (Figure 3). This phenomenon is highlighted by the observation that when comparing metal coatings to these uncoated particles, only an ionic zinc coating offered additional benefit (but not iron or silver).

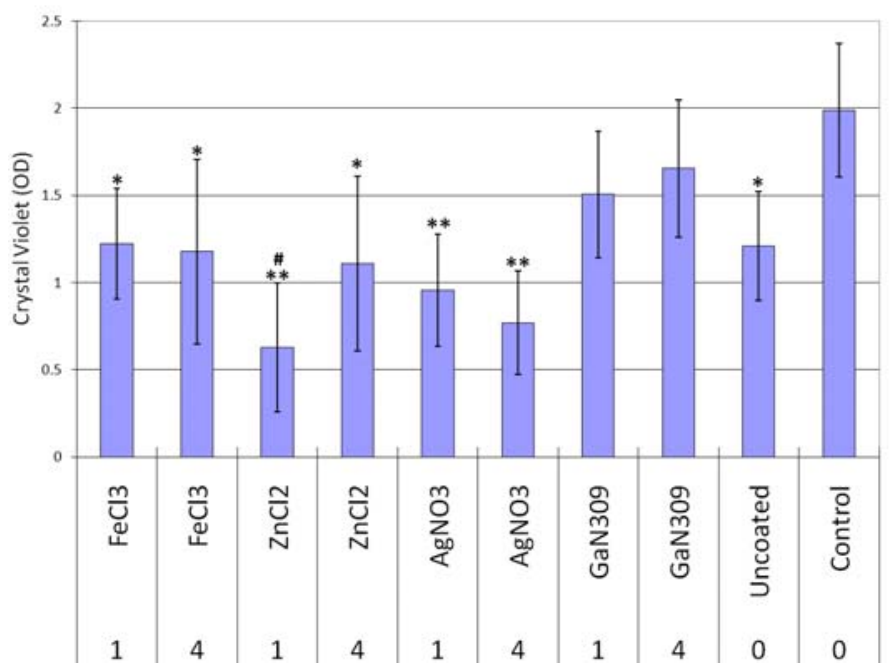

Figure 3. Biofilm inhibition of Staphylococcus aureus cultured in the presence of DMSA-SPION quantified by crystal violet. Bacteria were significantly inhibited by all DMSA-SPION (1 $\mathrm{mg} / \mathrm{ml}$ ) formulations (1 or 4 indicates mmol of salt added) after 48 hours, except gallium (Ga). *P values are significant at the $5 \%$ and $* * \mathrm{P} 1 \%$ level compared to control values $(\mathrm{N}=3)$. \#P value is significant at the $5 \%$

\section{CONCLUSIONS}

In this study, the possible minimal invasive treatment of device related infection was examined through the use of SPION. Results demonstrated that DMSA-SPION coated with silver were able to inhibit bacterial growth for 48 hours. Antibacterial properties of zinc were not observed possibly because the ion was not able to associate with the DMSA coating agent. In conclusion, these results help to confirm the hypothesis that SPION could have multiple uses during device related infections.

\section{ACKNOWLEDGEMENT}

The authors would like to thank the Hermann Foundation and NSF GK-12 for financial support.

\section{REFERENCES}

[1] R.M. Donlan, "Biofilms and Device-Associated Infections," Emerging Infectious Diseases 2001.

[2] L. Bernard et al. "Trends in the treatment of orthopaedic prosthetic infections" Journal of Antimicrobial Chemotherapy 2003.

[3] E. Klein et al., "Hospitalizations and Deaths Caused by MethicillinResistant Staphylococcus aureus,” Emerging Infectious Diseases 2007. 


\title{
Effect of Coupling Electrical Stimulation and Anodized Nanotubular Titanium for Orthopedic Applications
}

\author{
B.Ercan and T.J. Webster \\ Brown University \\ 182 Hope Street \\ Providence, RI 02912
}

\begin{abstract}
The limited ability of titanium implants to remain bonded to juxtaposed bone, the extensive callus (fibrous tissue) formation surrounding such implants and the microbial infection that might lead to amputation in severe cases are major reasons leading to orthopedic implant failure. To improve the cytocompatibility properties of titanium, nanotechnology has been used to create nanotopographical features (through anodization) on titanium. Additionally, electrical stimulation has been investigated in orthopedics to heal bone non-unions and fractures. In this study, electrical stimulation was combined with anodized nanotubular titanium to further improve implant function. To investigate this, osteoblast (OB; bone-forming cell), fibroblast (FB; fibrous tissue forming cells) and Staphyloccocus aureus ( $S$. aureus functions were determined on anodized nanotubular titanium under various voltages for up to 21 days of in vitro culture.
\end{abstract}

\section{INTRODUCTION}

Titanium has been extensively used in orthopedic applications as an implant material. In spite of the excellent mechanical and chemical properties of titanium that can benefit in orthopedics, the long-term success of titanium implants is not sufficient. One of the most promising techniques to enhance the cytocompatibility of titanium is anodization, which creates nanofeatured surfaces mimicking the topography of bone. In the past, it has been shown that $\mathrm{OB}$ functions were enhanced on surfaces containing has nanotopography [1]. Additionally, electrical stimulation is another approach to promote bone regeneration. It has been clinically used to accelerate the healing of spinal fusions, osteotomies, delayed fractures and non-unions in the bone. Although it is well known that electrical stimulation can enhance bone cell proliferation and functions in vivo and in vitro for conventional titanium implants, no work has been completed regarding long term osteoblast functions on anodized nanotubular titanium upon electrical stimulation. Therefore, in this study, conventional and anodized nanotubular titanium samples were compared for their ability to promote osteoblast functions, callus formation and its bactericidal effect on the wound when coupled with an optimized voltage window.

\section{METHODS}

99.2\% pure titanium foils were anodized using $20 \mathrm{~V}$ DC current for 6 minutes with a $1.5 \%$ HF electrolyte solution and a platinum cathode. The surfaces were characterized using scanning electron microscopy. OB (ATCC 11372, p\#8-15) were cultured in DMEM supplemented with 10\% FBS and 1\% $\mathrm{P} / \mathrm{S}$ and FB (ATCC 1213, p\#6-12) were cultured in EMEM supplemented with $10 \%$ FBS under standard culture conditions. The total seeding density for co-culture experiments was 1500 cells $/ \mathrm{cm}^{2}(750 / 750)$. For the electrical stimulation experiments, bipolar pulses $(0.4 \mathrm{~ms}$ pulse duration and $20 \mathrm{~Hz}$ frequency) were used each day for 1 hour. The voltages used for these experiments were $1,5,10$ and $15 \mathrm{~V}$, corresponding to $0.3,1.4,2.8$ and $4.2 \mathrm{~A} / \mathrm{m}^{2}$.

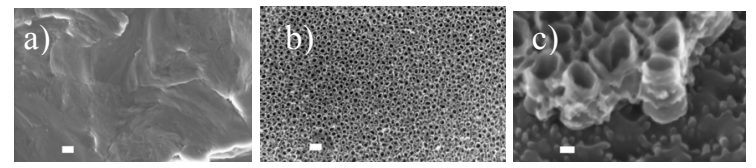

Figure 1: SEM images showing the surface features of a) conventional, b) anodized nanotubular and c) cross-section of the anodized nanotubular titanium. Scale bars are a) and b) $200 \mathrm{~nm}, \mathrm{c)} 30 \mathrm{~nm}$.

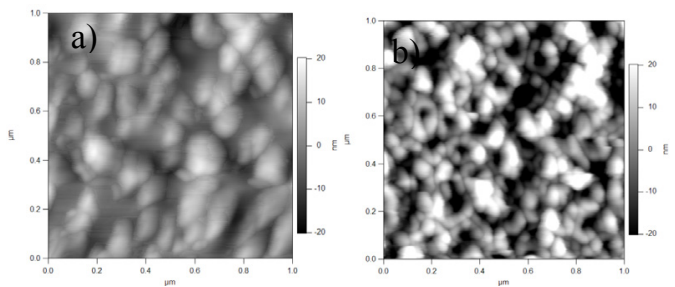

Figure 2: AFM micrographs showing the surface topography of a) conventional and b) anodized nanotubular titanium.

S. Aureus (ATCC 25923) was cultured using Luria broth (LB) under standard cell conditions using $200 \mathrm{rpm}$ agitation. For all experiments, bacteria were seeded on the substrates at a density of $1 \times 10^{7}$ bacteria/mL (as estimated by the McFaland scale) by diluting the LB bacteria cultures to an optical density of 0.52 at $562 \mathrm{~nm}$. The proliferation experiments were performed up to 3 days under standard cell culture conditions and constant shaking at $200 \mathrm{rpm}$. All results were analyzed using the standard analysis of variance techniques. 